Florida International University FIU Digital Commons

6-28-2013

\title{
Enhanced 3-Dimensional Carbon Nanotube Based Anodes for Li-ion Battery Applications
}

Chi Won Kang

Florida International University, ckang001@fiu.edu

DOI: $10.25148 /$ etd.FI13080904

Follow this and additional works at: https://digitalcommons.fiu.edu/etd

Part of the Materials Science and Engineering Commons

\section{Recommended Citation}

Kang, Chi Won, "Enhanced 3-Dimensional Carbon Nanotube Based Anodes for Li-ion Battery Applications" (2013). FIU Electronic Theses and Dissertations. 955.

https://digitalcommons.fiu.edu/etd/955

This work is brought to you for free and open access by the University Graduate School at FIU Digital Commons. It has been accepted for inclusion in FIU Electronic Theses and Dissertations by an authorized administrator of FIU Digital Commons. For more information, please contact dcc@fiu.edu. 


\title{
FLORIDA INTERNATIONAL UNIVERSITY
}

Miami, Florida, USA

\section{ENHANCED 3-DIMENSIONAL CARBON NANOTUBE BASED ANODES \\ FOR LI-ION BATTERY APPLICATIONS}

\author{
A dissertation submitted in partial fulfillment of the \\ requirements for the degree of \\ DOCTOR OF PHILOSOPHY \\ in \\ MATERIALS SCIENCE AND ENGINEERING \\ by \\ Chi Won Kang
}

2013 


\section{To: Dean Amir Mirmiran}

College of Engineering and Computing

This dissertation, written by Chi Won Kang, and entitled Enhanced 3-Dimensional Carbon Nanotube Based Anodes for Li-ion Battery Applications, having been approved in respect to style and intellectual content, is referred to you for judgment.

We have read this dissertation and recommend that it be approved.

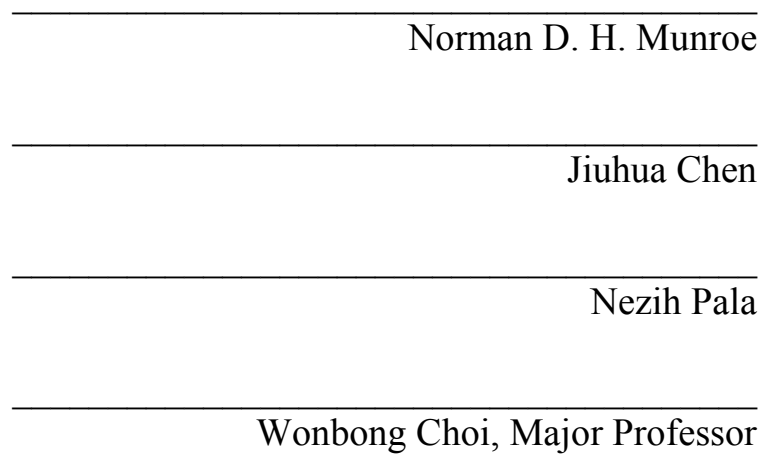

Date of Defense: June 28, 2013

The dissertation of Chi Won Kang is approved.

Dean Amir Mirmiran College of Engineering and Computing

Dean Lakshmi N. Reddi University Graduate School

Florida International University, 2013 
(C) Copyright 2013 by Chi Won Kang

All rights reserved. 


\section{DEDICATION}

I would like to dedicate this dissertation to Jesus Christ as my Savior and Lord for His measureless, strong and everlasting love.

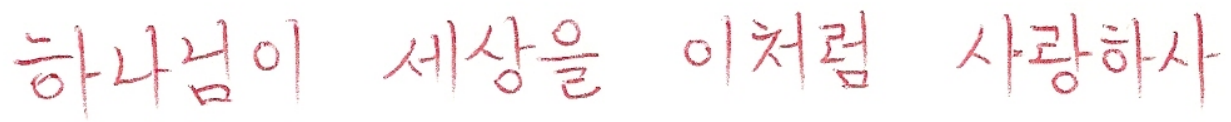

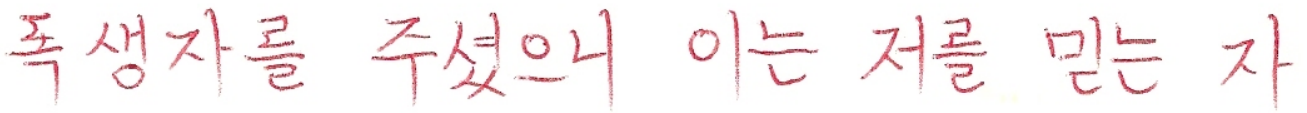

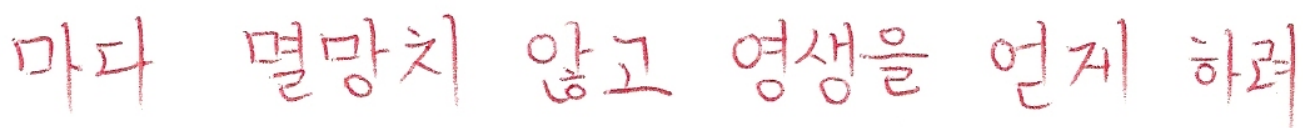

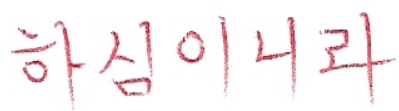

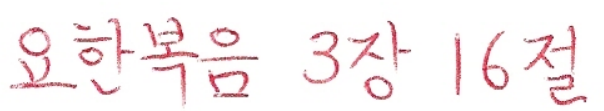

"For God so loved the world, that he gave his only begotten Son, that whosoever believeth in him should not perish, but have everlasting life."

John 3:16 (King James Version)

(translated from Korean to English) 


\section{ACKNOWLEDGMENTS}

I would like to express my sincere gratitude to my committee members, Prof. Wonbong Choi, Prof. Jiuhua Chen, Prof. Norman D. H. Munroe, and Prof. Nezih Pala for their continuous support and encouragement. My foremost thanks go to my dissertation advisor Prof. Wonbong Choi who led me towards an exciting area of my career with his insights and suggestions that helped to shape my research skills. I am also grateful to Prof. Choi for providing me opportunities during my studies at Florida International University (FIU).

I wish to express my warm and sincere thanks to Prof. Cesar Levy, chair of department of mechanical and materials engineering, who helped me during my $\mathrm{Ph} . \mathrm{D}$ program. I am also thankful to Dr. Rangasamy Baskaran at Hanyang University in Korea and Dr. Indranil Lahiri for collaborating with me for my experiments and research. Many thanks go to Prof. W. K. Jones, Dr. Y. Liu, Mr. Neal Ricks, and Dr. Santanu Das for their teaching and training to use cleanroom facility at Advanced Materials Engineering Research Institute (AMERI) in FIU. I want to thank Prof. S. Saxena for allowing me to use the Raman facility in The Center For The Study of Matter at Extreme Conditions (CeSMEC), and Prof. Y. K. Sun for his collaborating with me for Li-ion battery cell fabrication and test. Also, many thanks go to Dr. J. Y. Hwang and Prof. R. Banerjee for HRTEM characterization. I am thankful to Dr. Sookhyun Hwang for her helpful discussion and electrochemical analysis. Special thanks go to my friends, lab members, and staff at the department of mechanical and materials engineering and AMERI who helped me throughout my research and study at FIU. In addition, it is grateful that Mr. Joseph Kim and Mr. David Kim who provided me with the schematic diagrams. This research was, in part, supported by World Class

University (WCU) program through the Korea Science and Engineering Foundation funded by the Ministry of Education, Science and Technology (R31-2008-000-10092) and AFOSR Grant (FA9550-09-1-0544). 
Personally, I am expressing my gratitude to my mother Mrs. Hyewon Kim dedicated to me by her endless love and support. Also, I am grateful to my sister Mrs. Ayoung Kang, my brotherin-law Dr. Wan Lee, and other family members. I am thankful to my friend Dr. Byul Hur for his precious advice on my personal problem. 


\section{ABSTRACT OF THE DISSERTATION \\ ENHANCED 3-DIMENSIONAL CARBON NANOTUBE BASED ANODES \\ FOR LI-ION BATTERY APPLICATIONS}

by

Chi Won Kang

Florida International University, 2013

Miami, Florida

Professor Wonbong Choi, Major Professor

A prototype 3-dimensional (3D) anode, based on multiwall carbon nanotubes (MWCNTs), for Liion batteries (LIB), with potential use in Electric Vehicles (EVs) was investigated. The unique 3D design of the anode allowed much higher areal mass density of MWCNTs as active materials, resulting in more amount of $\mathrm{Li}^{+}$ion intake, compared to that of a conventional $2 \mathrm{D}$ counterpart. Furthermore, 3D amorphous Si/MWCNTs hybrid structure offered enhancement in electrochemical response (specific capacity $549 \mathrm{mAhg}^{-1}$ ). Also, an anode stack was fabricated to further increase the areal or volumetric mass density of MWCNTs. An areal mass density of the anode stack $34.9 \mathrm{mg} / \mathrm{cm}^{2}$ was attained, which is $1,342 \%$ higher than the value for a single layer $2.6 \mathrm{mg} / \mathrm{cm}^{2}$. Furthermore, the binder-assisted and hot-pressed anode stack yielded the average reversible, stable gravimetric and volumetric specific capacities of $213 \mathrm{mAhg}{ }^{-1}$ and $265 \mathrm{mAh} / \mathrm{cm}^{3}$, respectively (at 0.5C). Moreover, a large-scale patterned novel flexible 3D MWCNTs-graphenepolyethylene terephthalate (PET) anode structure was prepared. It generated a reversible specific capacity of $153 \mathrm{mAhg}^{-1}$ at $0.17 \mathrm{C}$ and cycling stability of $130 \mathrm{mAhg}^{-1}$ up to 50 cycles at $1.7 \mathrm{C}$. 


\section{TABLE OF CONTENTS}

CHAPTER

PAGE

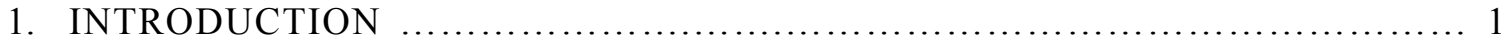

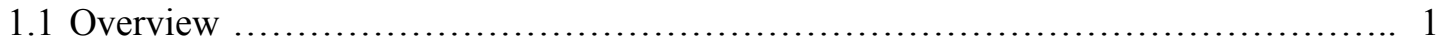

1.2 Motivation and Goal ............................................................ 7

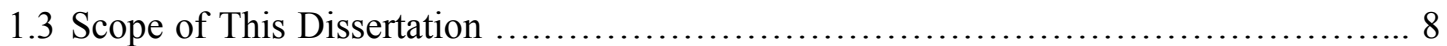

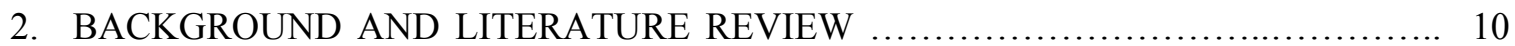

2.1 Lithium Ion Batteries ....................................................... 10

2.2 Major Types of Reaction Mechanism and Promising Active Materials for Anode ...... 16

2.2.1 Formation Reactions in Reconstitution Reactions .............................. 16

2.2.2 Displacement Reaction in Reconstitution Reactions .......................... 18

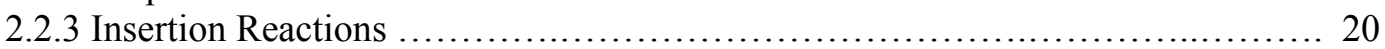

2.3 Brief Introduction to Carbon Nanotubes .............................................. 23

2.4 Carbon Nanotubes for Li-ion Battery Anode ...................................... 26

2.5 Hybrid Anode Structures for High-Performance Li-ion Batteries ........................ 31

2.6 3D Anode Architecture for High Loading Density of Active Materials .................. 32

2.7 Flexible Li-ion Batteries for Next-Generation Flexible Electronic Devices ............... 35

3. SYNTHESIS OF MWCNT DIRECT GROWN ON METAL SUBSTRATES AND BUTTON CELL ASSEMBLY COMPOSED OF MWCNT-BASED ANODE ...................... 39

3.1 Ti/Ni Thin Film Deposition on Cu Substrates by Using a RF Magnetron Sputter ...... 39

3.2 CNT Growth by Using a Catalytic Thermal Chemical Vapor Deposition (CVD) ....... 43

3.2.1 Chemical Vapor Deposition (CVD) ..................................... 43

3.3 Button Cell Assembly Under Ar Gas Flow Glove Box ............................... 53

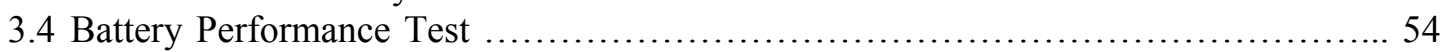

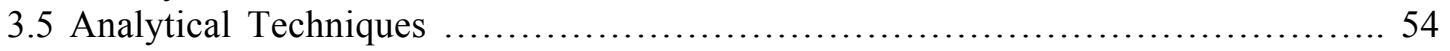

4. 3-DIMENSIONAL CARBON NANOTUBE FOR LI-ION BATTERY ANODE ......... 56

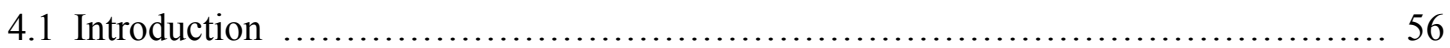

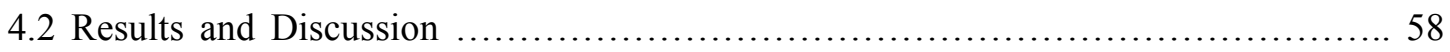

4.2.1 Experimental Details ................................................. 58

4.2.2 Theoretical Calculation of the Surface Area of 3D Cu Mesh .................... 59

4.2.3 Morphology and Structural Properties of MWCNTs and a-Si/MWCNTs on 3D Cu

Mesh .......................................................................... 67

4.2.4 Electrochemical Performances of the MWCNT-Based Anode Systems .......... 69

4.2.4.1 Charge/Discharge Capacities .................................. 69

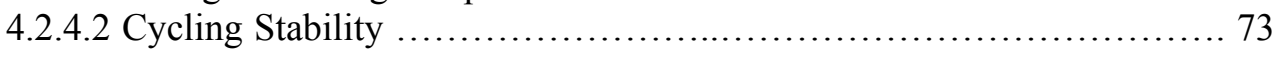

4.2.4.3 Rate Capability .................................................... 77

4.3 Conclusion .................................................................... 78

5. 3D ANODE STACK ASSEMBLED BY MULTIWALL CARBON NANOTUBES

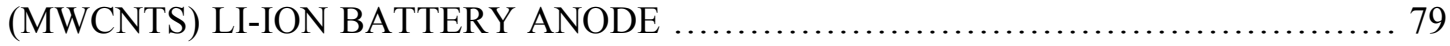

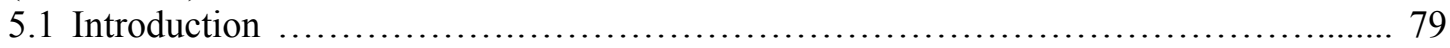

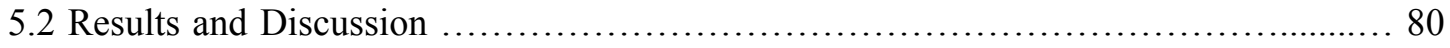

5.2 .1 Experimental Details ................................................. 80

5.2.2 Morphology and Structural Properties of MWCNT-Based Anode Stack .......... 83 
5.2.3 Electrochemical Properties of MWCNT-Based Anode Stack

6. LARGE SCALE PATTERNABLE 3D CARBON NANOTUBE-GRAPHENE STRUCTURE

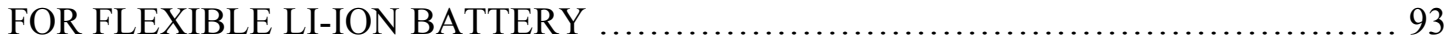

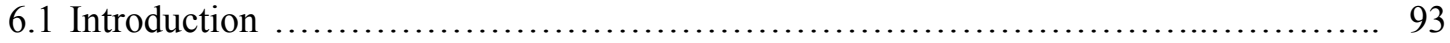

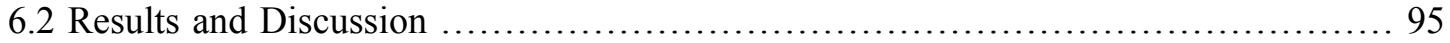

6.2.1 Experimental Details ............................................... 95

6.2.2 Structural and Electrical Properties of 3D MWCNTs-Graphene-PET ............. 100

6.2.3 Li-ion Battery Performance of 3D MWCNTs-Graphene-PET ................ 107

6.3 Conclusion ................................................................. 110

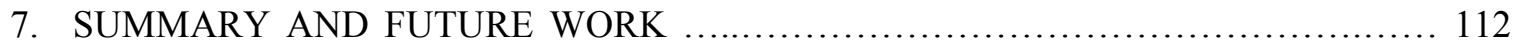

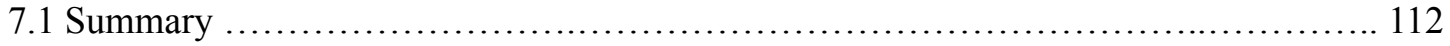

7.2 Future Scope of This Work ....................................................... 114

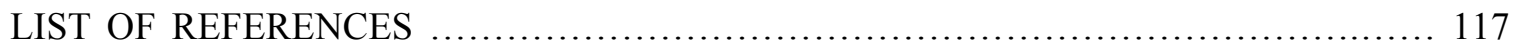

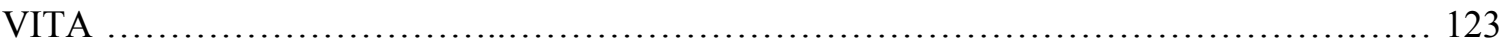




\section{LIST OF TABLES}

TABLE

PAGE

Table I. Main components and the examples of commonly used materials [53] ............... 12

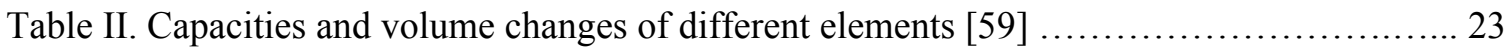

Table III. Standard process conditions used for Ti and Ni thin film deposition ................ 42

Table IV. The summary of key conditions for CNT growth through a catalytic thermal CVD

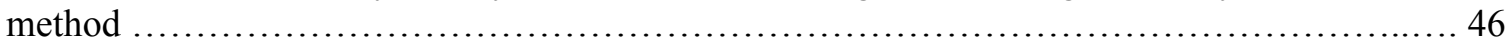

Table V. The variation of diameter of catalyst Ni islands with the CNT growth conditions. Ethylene gas as carbon precursor was flowed into the chamber for one minute. Numerical values noted in the top-left of each image represent the most probable size of the Ni islands .......... 48

Table VI. The variation of geometry and morphology of MWCNTs on 3D Cu mesh with their

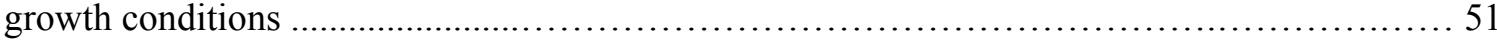




\section{LIST OF FIGURES}

FIGURE

PAGE

Figure 1. Diagram comparing the rechargeable battery technologies as a function of volumetric and specific energy densities. The arrows indicate the direction of development to reduce battery

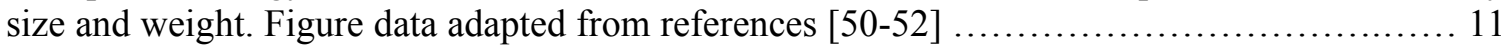

Figure 2. (a) Photograph of a conventional lithium ion battery active layer stack, including a $\mathrm{LiCoO}_{2}$ cathode composite on aluminum, microporous poly(olefin) separator, and MCMB anode composite on copper. Cross-sectional scanning electron micrographs depict the microstructure for

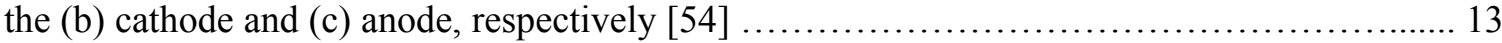

Figure 3. Schematic illustrating the mechanism of operation for a lithium ion battery, including the movement of ions between electrodes (solid lines) and the electron transport through the complete electrical circuit (dashed lines) during charge (blue) and discharge (red) states. Figure 3 adapted from reference [51]

Figure 4. (a) Cyclic voltammogram for Si NWs from $2.0 \mathrm{~V}$ to $0.01 \mathrm{~V}$ versus $\mathrm{Li} / \mathrm{Li}^{+}$at $1 \mathrm{mV} \mathrm{s}{ }^{-1}$ scan rate. The first seven cycles are shown. (b) Voltage profiles for the first and second galvanostatic cycles of the Si NWs at the $\mathrm{C} / 20$ rate. The first charge achieved the theoretical capacity of 4,200 $\mathrm{mAh} \mathrm{g}^{-1}$ for $\mathrm{Li}_{4.4} \mathrm{Si}$. (c) The voltage profiles for the Si NWs cycled at different power rates. The $\mathrm{C} / 20$ profile is from the second cycle. (d) Capacity versus cycle number for the Si NWs at the $\mathrm{C} / 20$ rate showing the charge (squares) and discharge capacity (circles). The charge data for Si nanocrystals (triangles) from reference [56] and the theoretical capacity for lithiated graphite (dashed line) are shown as a comparison to show the improvement when using Si NWs [16] 17

Figure 5. Properties of $\mathrm{MO} / \mathrm{Li}$ cells. (a) The voltage vs. composition profile for various $\mathrm{MO} / \mathrm{Li}$ cells cycled between $0.01 \mathrm{~V}$ and $3 \mathrm{~V}$ at a rate of $\mathrm{C} / 5$ (1 lithium in 5 hours). The capacity fading for the same cells under similar conditions are shown in (b), where we also show data for a $\mathrm{Co}_{3} \mathrm{O}_{4} / \mathrm{Li}$ cell in order to show that the reported behavior is not specific to divalent oxides. Inset, the rate capability of a $\mathrm{CoO}$ electrode [18] 19

Figure 6. Crystal structure of hexagonal graphite showing the $A B$ layer stacking sequence, the unit cell, and the subdivision in basal plane and prismatic surfaces [57] ................... 21

Figure 7. Diagram illustrating the lithium ion capacity and electrochemical reduction potentials with respect to lithium metal for conventional anode (red axis) and cathode materials (blue axis). The battery potential is the relative difference between the voltage of the selected positive electrode materials (blue ovals) and voltage of the corresponding negative electrode material (red ovals). Figure data adapted from references [51-52, 60-61] 24

Figure 8. Carbon nanotubes imaged by transmission electron microscopy (TEM). TEM is a technique used in the discovery of both MWNTs and SWNTs. $(a-c)$ TEM images of MWNTs with closed caps (courtesy of S. Iijima). The parallel lines are the cross-sections of the sidewalls of concentric cylinders. Diameters of MWNTs are typically on the order of 10-20 nm. (d) TEM image of the cross-section of a bundle of SWNTs courtesy of R. Smalley). Each circle represents the cross-section of a SWNT with diameter $\sim 1.4 \mathrm{~nm}[62]$ 
Figure 9. Chirality chart illustrating the assignment of $(n, m)$ SWCNT structures using a role-up vector from an origin point on a graphene sheet and the nanotube diameter [54] $\ldots \ldots \ldots \ldots \ldots \ldots .26$

Figure 10. Galvanostatic insertion-extraction of lithium into carbon nanotubes. Current load of 20

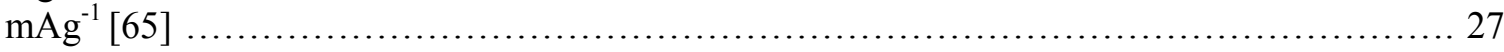

Figure 11. Electrochemical characteristics of the proposed CNT-based electrode structure. (a) First two charge-discharge cycles of the MWNT-on- $\mathrm{Cu}$ anode, at $0.1 \mathrm{C}$ rate. The first discharge cycle (lithiation) has shown a very high capacity of $2,547 \mathrm{mAhg}^{-1}$, while the following delithiation cycle showed a specific capacity of $1,455 \mathrm{mAhg}^{-1}$. (b) Reversible capacity of the MWNT-on-Cu anode, at different C-rates. Very high specific capacity could be observed at all Crates. Even at the $3 \mathrm{C}$ rate, a reversible capacity of $767 \mathrm{mAhg}^{-1}$ was observed, which is almost 2 times the theoretical capacity of graphite. (c) Exceptional stability of the reversible capacity $\left(\sim 900 \mathrm{mAhg}^{-1}\right)$ of the MWNT-on-Cu anode in long-run, at $1 \mathrm{C}$ rate. Except for the first two cycles, virtually no capacity degradation was observed for this anode structure, in 50 cycles. (d) Coulombic efficiency of the MWNT-on-Cu anode, showing very high efficiency, except for the first cycle. After the initial five cycles, the efficiency remained more than $99 \%$ [34].

Figure 12. A schematic (not to scale) of the proposed anode structure, showing its advantageous features [34] 30

Figure 13. (a) Sputtered-on Si film tends to pulverize after $\mathrm{Li}^{+}$cycling. (b) CNT-Si films can

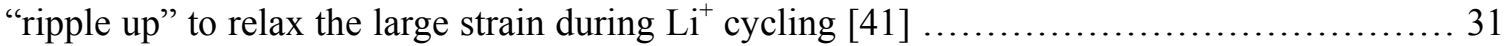

Figure 14. (a) Photograph of a free-standing CNT-Si film. (b) Charge (red) and discharge (green) capacity and Coulombic efficiency (blue) versus cycle number for a half cell using free-standing CNT-Si films as the working electrode cycled between 1 - $0.01 \mathrm{~V}$ [41] 32

Figure 15. One way to compare electrical energy storage devices is to use Ragone plots, which show both power density (speed of charge and discharge) and energy density (storage capacity). These plots for the same electrochemical capacitors are on a gravimetric (per weight) basis in (A) and on a volumetric basis in (B). The plots show that excellent properties of carbon materials will not translate to medium- and large-scale devices if thin-film and/or low-density electrodes are used [36] 33

Figure 16. Bicontinuous electrode fabrication process. The electrolytically active phase is yellow and the porous metal current collector is green. The electrolyte fills the remaining pores [38]

Figure 17. Lithium-ion battery ultrafast charge behavior. Potentiostatic charging at $3.6 \mathrm{~V}$ for $60 \mathrm{~s}$ (blue), $120 \mathrm{~s}$ (green) and $800 \mathrm{~s}$ (red), and $\sim 3 \mathrm{C}$ galvanostatic discharging of the prototype lithiumion pouch battery after each charging cycle [38] 35

Figure 18. Fabrication of the nanocomposite paper units for supercapacitor and battery. (a) Schematic of the supercapacitor and battery assembled by using nanocomposite film units. The nanocomposite unit comprises RTIL $([\mathrm{bmIm}][\mathrm{Cl}])$ and MWNT embedded inside cellulose paper. A thin extra layer of cellulose covers the top of the MWNT array. Ti/Au thin film deposited on the exposed MWNT acts as a current collector. In the battery, a thin Li electrode film is added onto the nanocomposite. (b) Photographs of the nanocomposite units demonstrating mechanical flexibility. Flat sheet (top), partially rolled (middle), and completely rolled up inside a capillary 
(bottom) are shown. (c) Cross-sectional SEM image of the nanocomposite paper showing MWNT protruding from the cellulose-RTIL thin films (Scale bar, $2 \mu \mathrm{m}$.). The schematic displays the partial exposure of MWNT [46]

Figure 19. Electrochemical measurements of nanocomposite paper battery. (a) First chargedischarge curves of the nanocomposite thin-film battery cycled between 3.6 and $0.1 \mathrm{~V}$ at a constant current of $10 \mathrm{~mA} / \mathrm{g}$. (b) charge capacity vs. number of cycles of the nanocomposite thinfilm battery. (c) The flexible nanocomposite film battery used to glow a red light-emitting diode (LED). The flexible battery consists of an individual nanocomposite thin film with the Li thin film present on one side as one of the electrodes. The LED glows even when the battery device is rolled up, and the demonstration could be repeated over several tens of cycles at an initial

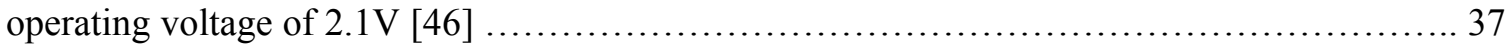

Figure 20. Schematic diagram of a simple magnetron sputtering system ................... 40

Figure 21. A three gun magnetron sputtering system (AJA International), used to synthesize

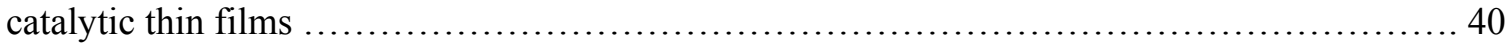

Figure 22. Schematic experimental setup for carbon nanotube growth by means of a catalytic thermal CVD method 43

Figure 23. Two general growth modes of nanotube in chemical vapor deposition. Left diagram: base growth mode. Right diagram: tip growth mode [75] 44

Figure 24. Thermal chemical vapor deposition systems manufactured by (a) FirstNano Inc., and (b) A-Tech system Inc. 46

Figure 25. The variation of the diameters of catalyst $\mathrm{Ni}$ islands and the areal weights of MWCNTs with CNT growth conditions

Figure 26. HRTEM images of the morphology of 3D MWCNTs synthesized at the different growth conditions designated by (a) S-CNT (C), (b) S-CNT (H), and (c) L-CNT .............. 52

Figure 27. (a) Plot of the CNT film growth rate vs. Fe thickness where three regions (I, II, and III) with different internal structures are observed. (b) A schematic of the internal structure transition from hollow to bamboolike [80]

Figure 28. Photograph images of (a) a glove box for LIB button cell assembly and (b)

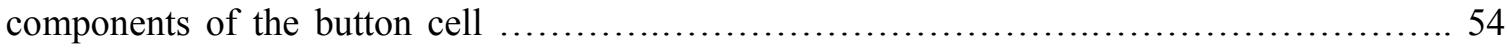

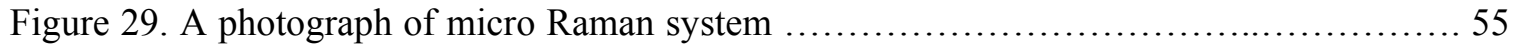

Figure 30. (a) A real SEM image of a 3D Cu mesh and the schematic diagrams (not to scale) to compare (b) the similar geometry of a 3D Cu mesh with (c) the simplified geometry of a 3D Cu

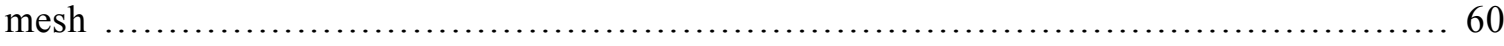

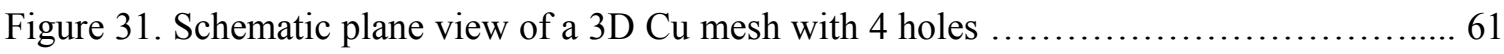

Figure 32. Schematic diagram of 3 dimensional view of a unit cell of a 3D Cu mesh ............ 62

Figure 33. The surface area and its increment of the $3 \mathrm{D} \mathrm{Cu}$ mesh and the $2 \mathrm{D} \mathrm{Cu}$ foil as a function of different thickness and hole sizes 
Figure 34. (a) A schematic model of an anode stack assembled using 4 numbers of converted uniform stacking cuboid arrays from the geometry of a real 3D Cu mesh (The bottom right inset shows a unit cell of the cuboid arrays.), (b) SEM images exhibiting a cross-section perpendicular to the anode system stacked by 9 individual MWCNTs on 3D $\mathrm{Cu}$ mesh and highly entangled structures of MWCNTs, (c) The surface area and its increment of the 3D Cu mesh and the 2D Cu foil and the average real weight of MWCNTs as a function of different thickness and hole sizes (The inset illustrates a unit cell of the $3 \mathrm{D} \mathrm{Cu}$ mesh with its dimension.) ...............66 66

Figure 35. Morphology and structure of the proposed anode systems. (a) A plane view of SEM image showing the MWCNTs covered on the 3D Cu mesh, (b) The threadlike grass structures of MWCNTs on the 3D Cu mesh with their diameter in the range of $200-300 \mathrm{~nm}$, (c) Schematic diagram (not to scale) of the geometry of the MWCNTs grown on the 3D $\mathrm{Cu}$ mesh and the a-Si deposited MWCNTs structure on the 3D Cu mesh, (d) EDS elemental analysis of the aSi/MWCNTs hybrid structure in the linearly selected area across the SEM image, (e) Raman spectroscopic response indicating high defect density of MWCNTs according to $\mathrm{I}_{\mathrm{D}} / \mathrm{I}_{\mathrm{G}}$ ratio around 1 and the amorphous $\mathrm{Si}$ peak at around $500 \mathrm{~cm}^{-1}$ in the a-Si/MWCNTs

Figure 36. Electrochemical performance of the anode structures of as-grown MWCNTs on 3D Cu mesh, MWCNTs on 2D Cu foil and a-Si/MWCNTs core-shell composite on 3D Cu mesh. (a) First charge-discharge cycle at $0.1 \mathrm{C}$-rate, (b) Comparison of the cycling stability of the different anode structures, (c) Coulombic efficiency at $1 \mathrm{C}$-rate, (d) The variation of reversible capacities at different $\mathrm{C}$-rates 70

Figure 37. Plane view SEM images of (a) the MWCNTs on 3D $\mathrm{Cu}$ mesh anodes after 150 cycling at $1 \mathrm{C}$-rate and (b) the MWCNTs after only 1 discharge cycling at the same C-rate. HRTEM images of (c) the 150 cycled one and (d) the 1 discharge cycled one ................ 76

Figure 38. Process flow for anode stack system from an individual 3D MWCNTs on $\mathrm{Cu}$ mesh current collector to 3D free-standing MWCNT anode stack system. (a) As-received commercially available 3D Cu mesh, (b) CVD grown 3D MWCNTs on the surface of the $\mathrm{Cu}$ mesh, (c) Chemical etching process of the $\mathrm{Cu}$ mesh via $\mathrm{FeCl}_{3}$ etching solution, (d) 3D free-standing MWCNT mesh structure after the etching process, (e) Fabrication of anode stack consisted of multiple layers of 3D free-standing MWCNT meshes by finally pressing them together ...... 82

Figure 39. Structural properties of anode stack composed of 3D MWCNTs presented by diverse analysis techniques. (a) SEM plane view of 3D free-standing MWCNT structure. Inset shows higher magnification SEM image of the thread-like MWCNT structure; (b) High resolution TEM (HRTEM) image of a single MWCNT; (c) SEM image indicating the point, denoted by the red cross, where $\mathrm{Cu}$ mesh originally existed before $\mathrm{Cu}$ etching process; (d) EDS spectra of 3D freestanding MWCNTs obtained from the spot indicated by the red cross shown in the SEM image of (c); (e) Raman spectra of 3D MWCNTs; (f) SEM image of the cross-sectioned MWCNT-based anode stack showing high areal mass density of MWCNTs around $34.9 \mathrm{mg} / \mathrm{cm}^{2}$; (g) The photographs of the final product of anode stack for its button cell assembly; (h) The crosssectional SEM image of the hot pressed anode stack

Figure 40. Electrochemical performance of the anode stacks with different areal loading density of 3D MWCNTs as active materials for LIB application. A primary charge and discharge profile of the anode stacks in terms of (a) gravimetric and (b) volumetric specific capacities at $0.05 \mathrm{C}$, Cycling stabilities of the two different anode stacks with respect to (c) gravimetric and (d) volumetric specific capacities at $0.5 \mathrm{C}$ 
Figure 41. Cross-sectional FESEM images of (a) mechanically compressed anode stack with the lower pressure and (b) thermal compressed anode stack with the higher pressure, (c-d) Plane-view FESEM micrographs taken from different regions of the thermal compressed anode stack with the higher pressure. The Insets show the magnified regions of the FESEM images to identify the morphology of MWCNT network structure

Figure 42. Process flow of transferring techniques of 3D MWCNTs/Cu mesh onto the graphene/ transparent flexible PET substrate. (a) Graphene transferred onto PET film, (b) 3D MWCNTs on $\mathrm{Cu}$ mesh directly grown by CVD, (c) Hot lamination of 3D MWCNTs/Cu mesh with graphene/PET, (d) Etching process of $\mathrm{Cu}$ mesh by using $\mathrm{FeCl}_{3}$ oxidation acid, (e) 3D MWCNTs pattern transferred onto the graphene-PET film. Inset shows a digital image of high flexibility of

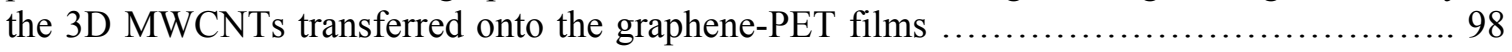

Figure 43. Digital images of the 3D CNFs/graphene-PET structure 99

Figure 44. Structural analysis of as-synthesized 3D MWCNTs-graphene-PET structure. (a) The magnified SEM image, (b) Zoomed-in SEM image showing detailed morphology of the structure, (c) The magnified SEM image of the randomly oriented CNF structures in the pattered structure, (d) Zoomed-in SEM cross sectional image, (e) Schematic diagram (not to scale) of the cross sectional image shown in Figure 2(d) ... 102

Figure 45. (a) 2 dimensional EDS mapping for the carbon distribution in Figure 44(a), (b) Line scanned EDS mapping according to the red colored arrow presented in Figure 44(a) showing the presence of the predominant carbon element, (c) EDS spectra of a spot of 3D MWCNTs on graphene-PET film showing higher than 99 atom\% carbon and less than 0.2 atom $\%$ of copper, (d) HRTEM image of the randomly oriented distribution of the graphene layer in a MWCNT

Figure 46. Variation in the normalized resistance $\left(\mathrm{R}_{\mathrm{deg}} / \mathrm{R}_{\mathrm{o}}\right)$ as a function of the angle of curvature 105

Figure 47. Raman spectra of (a) 3D MWCNTs and graphene on two different substrates Cu foil and PET film and pristine PET film and (b) magnified Raman spectra of graphene on PET and pristine PET film 106

Figure 48. Electrochemical performance of the 3D MWCNTs-graphene-PET anode. (a) Initial Galvanostatic charge and discharge profiles of 3D MWCNTs-graphene-PET film anode structure, (b) Cycling performance of the anode in higher number of cycles, (c) Coulombic efficiency of the anode at $1.7 \mathrm{C}$, (d) C-rate capability of the anode at seven different current densities. Numerical values noted in (d) indicate current densities, at which charge and discharge cycles were conducted 109 


\section{CHAPTER 1}

\section{INTRODUCTION}

\subsection{Overview}

Energy storage is unquestionably one of the most significant research topics in the twentyfirst century [1]. Technological and social developments of humankind have been supported by the discovery of new sources of energy and its proper application in human lives [2]. Importantly, the transportation industry has used fossil fuels as a main source of energy. However, both world impending oil shortage and global warming caused by green-house gases like $\mathrm{CO}_{2}$ are responsible for shifting the focus of power generation towards clean and carbon-free resources [3]. One essential and challengeable shift has been the electric vehicles (EVs) in which the motive power is supplied by the use of a battery. We expect the advent of EV markets based on the rapid increase in sales of hybrid electric vehicles $\left(\mathrm{HEVs}^{1}\right)$ [4]. Among various energy storage devices (e.g., nickel-cadmium battery or nickel-metal hydride battery), a lithium-ion battery (LIB) is one of the most commonly used devices for portable electronic devices and hybrid electric vehicles in modern human life [5]. Since the commercial release of LIBs by Sony in 1991 [6], Li-ion batteries (LIBs) have continued to attract great attention from many researchers around the world due to their many advantages. LIBs exhibit higher operation voltage greater than $3 \mathrm{~V}$, their capability to balance specific capacity with the capability of charge and discharge rate (i.e. C-rate capability), compared to other electrochemical energy storage devices. Additionally, LIBs provide higher energy density, broader temperature range, lower self-discharge rate, and no voltage depression (i.e. "memory effect"), relative to other energy storage devices mentioned

\footnotetext{
${ }^{1}$ A relative small internal combustion engine is used to charge a modest-sized electric battery, which then acts to provide the vehicular propulsion
} 
above. Such major advantages of LIBs make it possible to yield smaller, lighter, and environmentally friendly LIB cells [7].

For instance, the markets of cell-phone, laptop PC and camcorder (3C) have been powered by LIBs which are currently produced by billions of units per year [8]. The components of an LIB cell, commercially available carbon-based anode materials, and their electrochemical performances will be dealt with in chapter 2. Although such LIBs are commercially successful, we are now reaching the limits in performance using the current electrode and electrolyte materials. Demand for next-generation high-efficiency and low-cost LIBs have created excitement in the scientific community to discover new avenues for breakthrough in electrode materials [9]. The application of nanomaterials to LIB is one avenue which is being closely analyzed by many scientists and researchers.

Nanostructured materials have attracted great interest in recent years due to their unusual mechanical, electrical and optical properties endowed by confining the dimensions of such materials, and because of the combination of bulk and surface properties to their overall behavior. Currently, nanostructured materials are becoming increasingly important for electrochemical energy storage. Therefore, it is important to appreciate the advantages and disadvantages of nanomaterials for energy conversion and storage, as well as how to control their synthesis and properties [9].

There are several potential advantages and disadvantages associated with the development of nanomaterials for LIB electrodes. Advantages include:

(i) Better accommodation of the strain of lithium insertion and extraction, improving cycle life of electrode system 
(ii) New reactions for higher charge and discharge capacities not possible with bulk materials

(iii) Higher electrode/electrolyte contact area leading to higher charge and discharge rates (C rates)

(iv) Short path lengths for electronic and $\mathrm{Li}^{+}$ion transport.

However, disadvantages include:

(i) An increase in undesirable electrode/electrolyte reactions due to the high surface area of nanostructures for electrode materials, leading to self-discharge, poor cycling and calendar life

(ii) Lower packing density of particles, leading to lower volumetric energy densities unless special compaction methods are employed

(iii) Potentially more complicated synthesis methods [9].

With these advantages and disadvantages in mind, efforts have been devoted to exploring nanomaterials for negative electrodes (anodes) for LIBs (e.g. carbon nanotubes (CNTs) [10], graphene [11], silicon ( $\mathrm{Si}$ ) nanowires [12], and transition metal oxide nanoparticles [13]). Metals that store lithium ions are among the most appealing and competitive candidates for new types of anodes in LIBs. Indeed, a number of metals and semiconductors, for example tin (Sn) and silicon (Si), react with lithium to form alloys by electrochemical processes that are partially reversible and of low voltage (relative to lithium), involve a large number of atoms per formula unit, and provide a specific capacity much larger than that offered by conventional graphite $\left(372 \mathrm{mAhg}^{-1}\right)$. Conversely, such alloy-based anode materials have a critical limitation, which is the pulverization due to larger expansion (300-400\%) during lithiation and delithiation processes, resulting in 
capacity fading in a high number of cycles [14]. To overcome such limitation of bulk scaled alloy-based materials, worldwide research groups have intensively focused on novel and suitable nanomaterials, such as silicon nanotubes [15] and silicon nanowires [16-17].

A decade ago, P. Poizot proposed the new mechanism of Li reactivity, which differs from the classical $\mathrm{Li}$ insertion/deinsertion or Li-alloying processes and involves the formation and decomposition of $\mathrm{Li}_{2} \mathrm{O}$, accompanying the reduction and oxidation of metal nanoparticles (in the range $1 \pm 5 \mathrm{~nm}$ ), respectively [18-19]. Electrodes made of nanoparticles of transition-metal oxides (MO, where $\mathrm{M}$ is $\mathrm{Co}, \mathrm{Ni}, \mathrm{Cu}$ or $\mathrm{Fe}$ ) demonstrated electrochemical capacities of $700 \mathrm{mAhg}^{-1}$, with $100 \%$ capacity retention for up to 100 cycles and high recharging rates (C-rate capability). Since then, many research groups have been involved in the new anode materials. B. Varghese et al. fabricated vertically aligned $\mathrm{NiO}$ nanowalls on nickel foils using a plasma-assisted oxidation method and reported their high specific capacity of $635 \mathrm{mAhg}^{-1}$ at $1.2 \mathrm{C}$ and excellent capacity retention around up to 85 number of cycles in the range of 0.005 to $3.0 \mathrm{~V}$ vs. $\mathrm{Li}$ [20]. However, those transition metal oxide-based anodes have some drawbacks, such as poor cycling stability and low electric conductivity.

In terms of carbonaceous anodes, graphite and soft or poorly ordered carbons such as mesocarbon microbeads (MCMB), spherical graphite and microcarbon fiber have been employed to be used as anode materials. The reasons behind their commercial prominence are the relatively low cost of carbon, the excellent mechanical sustainability for lithium insertion and desertion (having minimum volume change, $\sim 12 \%$ ) and the formation of a protective surface film (e.g. SEI layer) with many different electrolytes [21-23]. Nevertheless, fully intercalated highly crystalline graphites have relatively lower specific capacity $\left(372 \mathrm{mAhg}^{-1}\right.$, the stoichiometric formulae of $\mathrm{LiC}_{6}$ ), and the level of the specific capacity will not be able to meet the demands of nextgeneration LIB with respect to high specific capacity and volumetric capacity. 
To address these issues, many research groups have been interested in multi-walled carbon nanotubes (MWCNTs) due to their unique multi-walled co-centric tube geometries, excellent electrical conductivity, wide electrochemical-stability window, highly accessible surface area, and short diffusion length of $\mathrm{Li}^{+}$ions $[4,24]$. It has been speculated that a higher Li capacity may be obtained in carbon nanotubes if all the interstitial sites (inter-shell van der Waals spaces, intertube channels, and inner cores) are accessible for $\mathrm{Li}$ intercalation [25]. Electrochemical intercalation of MWCNTs [26-31] and SWCNTs [32-33] has been investigated by several groups.

Importantly, electrochemical performances were obtained from CNT-based composites composed of PVDF $\left(-\left(\mathrm{C}_{2} \mathrm{H}_{2} \mathrm{~F}_{2}\right)_{\mathrm{n}^{-}}\right)$as binder and carbon nanopowders, as conductive additives dissolved in organic solvents. On the other hand, the most commonly used binder PVDF tends to form compounds with graphite and Li metal. For instance, the high energy per gram of PVDF $(7.2 \mathrm{~kJ} / \mathrm{g})$ is generated by its compound formation of Li and brings about safety issues such as possible explosions. Also, because of that, high-temperature utilization of Li-ion batteries could not be available. Therefore, it is necessary to design a novel anode system.

In the past, our research team demonstrated outstanding performance of MWCNT-based binder-free anodes in terms of high specific capacity, excellent rate capability and extremely low or nil capacity degradation during long cycle operation [34-35]. However, carbon nano-materials are known as low-density materials that result in low volumetric capacity and low volumetric energy/power density. Therefore, higher solid loading of MWCNTs as active materials is one of the most significant issue to be realized in practice. Very recently, it was argued that nanotubebased active materials have a critical shortcoming in terms of their very low weight per unit electrode area [36]. Thus, their gravimetric energy density may not provide a realistic picture of their commercial application. This critical limitation may lead to scale-up issues for their potential application in the development of EVs. 
To counter this issue, we propose a new geometry of 3D $\mathrm{Cu}$ current collectors, which can play a crucial role in creating higher surface area to accommodate more solid loading of MWCNTs on the uniformly arrayed patterns in the 3D structure, leading to higher specific capacity and C-rate capability. Until now, efforts have been dedicated to employ a number of 3D structured current collectors, including carbon papers [37], a self-assembled 3D bicontinuous nanoarchitecture [38], aluminum nanorods [39], and nanoporous nickel [40]. The previous research proved that a self-assembled 3D bicontinuous nanoarchitecture could be one of the ideal electrode architectures in order to realize not only the high volume fraction of nanostructured electrolytically active materials ( $\mathrm{NiOOH} / \mathrm{Nickel}$ and $\mathrm{MnO}_{2}$ cathodes) but also their efficient ion and electron transport [38]. In addition, atomic layer deposition (ALD) coated $\mathrm{TiO}_{2}$ anodes on $3 \mathrm{D}$ aluminum nanorod current collectors showed a tenfold increase in their theoretical area and total capacity $\left(0.0112 \mathrm{mAhcm}^{-2}\right)$, compared to those results from the same anodes on 2D flat aluminum plate and high-rate capability (the capacity ratios at $10 \mathrm{C} / 0.5 \mathrm{C}$ and $20 \mathrm{C} / 0.5 \mathrm{C}$ of the 3D anode were 0.4 and 0.35 , respectively.) [39].

Currently, the diverse types of hybrid anode structures have been designed and synthesized in order to expect the synergetic combination of two different types of nanomaterials for higher electrochemical performance. As one of the most preferable combinations, MWCNTs/Si hybrid structure can be chosen due to better mechanical accommodation of MWCNTs of the large volume expansion/constriction of Si during the lithiation/delithiation process and higher bonding strength between MWCNTs and $\mathrm{Si}$. There are several reports on MWCNTs/Si composite structures; however, data employing either the $\mathrm{SiH}_{4} \mathrm{CVD}$ method [41] or sputter deposition [42] were used for this dissertation, specifically.

Furthermore, we investigated a new approach which is stacking multi-layers of 3D MWCNTs to increase an area or volumetric mass density of 3D MWCNT based anode materials. Previously, 
L. Hu et al. demonstrated the feasibility of novel anode systems and multi-layers stacked with the $\mathrm{CNT} / \mathrm{Li}_{4} \mathrm{Ti}_{5} \mathrm{O}_{12}(\mathrm{LTO}) /$ paper/ $/ \mathrm{LiCoO}_{2}(\mathrm{LCO}) / \mathrm{CNT}$ structure in order to increase the total energy density of flexible LIBs [43]. With the anode stack, the authors achieved similar electrochemical performance to that of an individual anode cell.

In addition, flexible electronics have recently attracted great attention to the next generation portable devices, such as roll/up displays, wearable devices, active radio frequency identification (RFID) tags, integrated circuit smart cards and implantable medical devices as their major applications. Flexible Li-ion batteries (LIBs) are used as the energy storage devices for the flexible electronics [44-45]. To date, many leading research groups have combined the nanomaterial-based LIB with the flexible polymer substrates. V. L. Pushparaj et al. fabricated a flexible nanocomposite thin-film battery composed of a CNT-cellulose paper electrode, which achieved a reversible capacity of $110 \mathrm{mAhg}^{-1}$ over several tens of cycles [46]. J. Chen et al. prepared CVD-grown CNT/carbon layer (CL) hybrid anodes for a paper LIB and demonstrated its high reversible capacity of $572 \mathrm{mAhg}^{-1}$ after 100 cycles [47]. M. Kaempgen et al. developed single-walled carbon nanotubes (SWCNTs) spray-deposited onto polyethylene terephthalate (PET, $\left(-\left(\mathrm{C}_{10} \mathrm{H}_{8} \mathrm{O}_{4}\right)_{\mathrm{n}^{-}}\right)$films and obtained high specific capacitances, ranging from $\sim 90$ to $120 \mathrm{~F} / \mathrm{g}$ by using various aqueous electrolytes [48].

\subsection{Motivation and Goal}

This dissertation focuses on the fabrication of various 3D MWCNT-based nanoarchitectures and the application of novel nanostructures for the anode of LIBs. As mentioned earlier, MWCNTs are suitable for high-potential anode materials because of their exceptional properties such as specific surface area, great flexibility, larger electron conductivity, and short diffusion 
length of lithium ions. In spite of these advantages, MWCNT based anode structures have a critical drawback, which is lower loading density.

To address this issue, this investigation is focused on the synthesis of a novel $3 \mathrm{D}$ anode structure which increases an area or volumetric mass density of 3D MWCNT-based active materials. In addition, it is aimed at attaining better electrochemical performance of the 3D structures, compared to that of conventional 2D structures (i.e. MWCNTs directly grown on 2D $\mathrm{Cu}$ ). Also, owing to the highest specific capacity $\left(4,200 \mathrm{mAhg}^{-1}\right)$, silicon-based nanostructured materials have attracted strong attention from researchers for LIB around the world. In the present work, amorphous silicon (a-Si)/3D MWCNT hybrid anode structures are presented to attain better LIB peformance. Furthermore, to appreciably increase an area or volumetric specific capacity of anodes, the fabrication of 3D MWCNT-based anode stack has been carried out as an important part of this dissertation.

Currently, the flexible display market is expected to grow fast by two big market sectors, which are e-paper technology-centered new diverse applications and the field of replacement markets of the conventional applications [49]. A flexible LIB as an important energy storage device has triggered strong excitement in the scientific community and extensive research worldwide. Regarding this issue, another goal is pursued, which is the preparation for a novel anode structure composed of 3D MWCNTs-graphene-PET flexible film yielding a good electrochemical performance.

\subsection{Scope of This Dissertation}

Chapter 2 provides the background of the dissertation and discusses the current technology of the synthesis of CNTs and their electrochemical properties for LIBs. The basic concept of LIBs 
and CNTs are described. The progress of the novel anode nanostructures and LIB performance are also discussed based on the current technology. A recent development in carbon nanostructure-based flexible electrodes has also been dealt with in this chapter. Chapter 3 deals with the synthesis process of MWCNTs through a two-step preparation method, including radio frequency (RF) magnetron sputtering and catalytic thermal chemical vapor deposition (CVD). Chapter 4 describes the fabrication of 3D MWCNTs directly grown on $\mathrm{Cu}$ current collector and the LIB performance with the anode system. This chapter mainly emphasizes the increased loading of MWCNT active materials and enhanced LIB properties compared with those of their 2D counterpart with MWCNTs grown on $\mathrm{Cu}$ foil. Furthermore, the improved LIB performance obtained with the anode system of a-Si/3D MWCNTs on $\mathrm{Cu}$ is investigated in terms of higher lithiation of amorphous silicon. Chapter 5 describes the fabrication of anode stacks composed of several MWCNT layers which increase the areal and volumetric loading density of MWCNTs and the characterization of LIB. The main purpose for preparation of the anode stack is strongly related to the real picture of the anode stack for its electric vehicle (EV) application which demands larger loading density of active materials for higher total power and energy density. Chapter 6 describes the synthesis of 3D MWCNTs and graphene film by CVD method and transfer of 3D MWCNTs and graphene over transparent and flexible polyethylene terephthalate (PET) films by hot press lamination and chemical etching. The LIB performance of 3D MWCNTs-graphene-PET film is also presented in this chapter. The structural integrity and electronic properties of the flexible 3D anode structure is significantly discussed, since they are important factors that are responsible for good LIB performance. Finally, chapter 7 provides a summary of the dissertation and proposes future work to be conducted. 


\section{CHAPTER 2.}

\section{BACKGROUND AND LITERATURE REVIEW}

\subsection{Lithium Ion Batteries}

Energy storage devices have attracted great attention for their technological applications, such as hybrid-electric-vehicles (HEVs) or full-electric-vehicles (EVs) in modern human life. The development of novel energy storage devices (ESDs) and their electrode materials is required to meet current social demand, such as impending global oil shortage and the need to preserve the ecosystem from severe green-house gas emission (e.g. $\mathrm{CO}_{2}$ ). Among the strong candidates of ESDs, Li-ion battery (LIB) has been intensively studied due to its $3 \mathrm{~V}$ magnitude of operation voltage and capability to enhance both specific energy and specific power, relative to other ESDs such as Ni-Cd or Ni-metal hydride battery.

Figure 1 represents the correlation between specific and volumetric energy densities for the major second rechargeable batteries. As seen in Figure 1, LIB shows both volumetric and specific energy densities, which can lead to the minimization of volume and mass of components in LIB. The specific energy released in the charge and discharge reaction is the product of the operation voltage and the specific capacity of the battery system. In the case of LIB, the operation voltage is around $3 \mathrm{~V}$ and many available anode materials exhibit high specific capacities (e.g. the theoretical specific capacity of Si is $4,200 \mathrm{mAhg}^{-1}$ ). The basics of the major anode materials are discussed in detail in section 2.2. Owing to those advantages of LIB, portable electronics (e.g. cellular phones, laptops, camcorders etc.) are mainly powered by LIB, and consumers favor smaller and lighter electronic devices that are fast charged and possess long battery life. Furthermore, such a balance between high specific energy and high charge rate has sparked a 
growing interest in the scientific communities, in particular among technologists and multidiscipline scientists exploring the way to incorporate lithium ion technology into next-generation EVs. Also, increased safety and appropriate cyclability are the main research goals of the nextgeneration and high-efficiency LIBs.

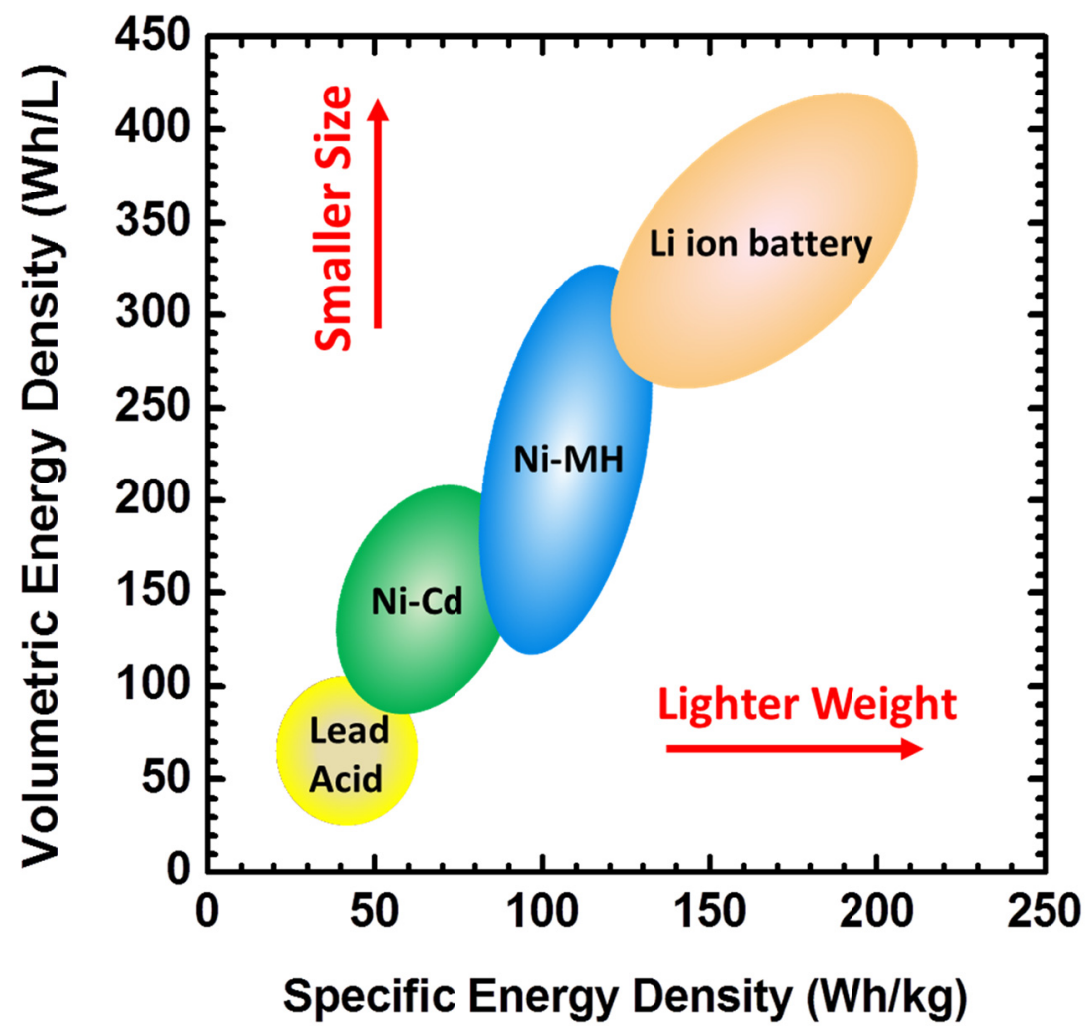

Figure 1. Diagram comparing rechargeable battery technologies as a function of volumetric and specific energy densities. The arrows indicate the direction of development to reduce battery size and weight. Figure data are adapted from references [50-52]. 
The electrochemical performance of LIB is strongly dependent on its component materials.

The commonly used components of LIB cells are listed in Table I.

Table I. Main components and the examples of commonly used materials [53]

\begin{tabular}{|c|c|c|}
\hline \multicolumn{2}{|r|}{ Components } & Commonly used materials \\
\hline \multirow{5}{*}{ Electrode } & Positive active materials & LiCoO2, LiMn2O4, LiNiO2, LiFePO4 \\
\hline & Negative active materials & $\begin{array}{l}\text { Graphite, hard(soft) carbon, Li, Si, Sn, } \\
\text { lithium alloy }\end{array}$ \\
\hline & Conductive additives & Acetylene black \\
\hline & Binder & PVdF, SBR/CMC \\
\hline & Current collector & $\mathrm{Cu}(-), \mathrm{Al}(+)$ \\
\hline \multirow{4}{*}{ Electrolyte } & Separator & $\begin{array}{l}\text { Polyethylene (PE), polypropylene (PP), } \\
\text { polyvinylidenefluoride (PVdF) }\end{array}$ \\
\hline & Lithium salt & $\begin{array}{l}\mathrm{LiPF}_{6}, \mathrm{LiBF}_{4}, \mathrm{LiAsF}_{6}, \mathrm{LiClO}_{4}, \mathrm{LiCF}_{3} \mathrm{SO}_{3} \text {, } \\
\mathrm{Li}\left(\mathrm{CF}_{3} \mathrm{SO}_{2}\right)_{2} \mathrm{~N}\end{array}$ \\
\hline & Electrolyte solvent & $\begin{array}{l}\text { Ethylene carbonate (EC), propylene } \\
\text { carbonate (PC), dimethyl carbonate } \\
\text { (DMC), diethyl carbonate (DEC), } \\
\text { Ethylmethyl carbonate (EMC) }\end{array}$ \\
\hline & Additives & $\begin{array}{l}\text { Vinylene carbonate (VC), biphenyl } \\
\text { (BP) }\end{array}$ \\
\hline
\end{tabular}

Figure 2(a) illustrates an active material stack composed of a cathode active material $\left(\mathrm{LiCoO}_{2}\right)$ coated on an aluminum current collector and an anode active material (mesocarbon microbeads $(\mathrm{MCMBs}))$ deposited onto a copper foil current collector, and a commercial separator is placed in between the cathode and the anode. Figure 2(b) and 2(c) are representative high magnification cross-sectional scanning electron micrographs of such cathode and anode composite electrodes, respectively [54]. 


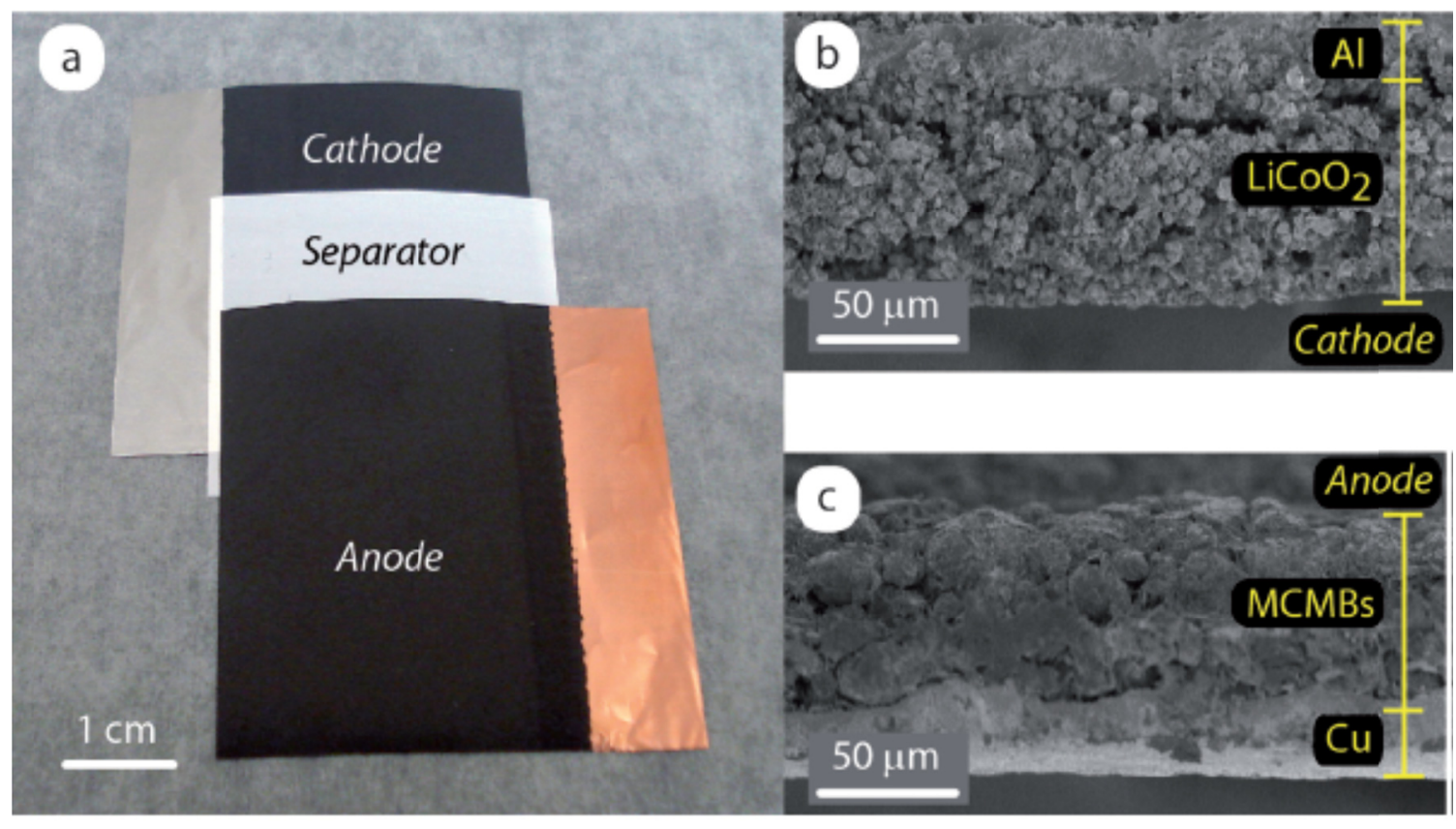

Figure. 2 (a) Photograph of a conventional lithium ion battery active layer stack, including a $\mathrm{LiCoO}_{2}$ cathode composite on aluminum, microporous poly(olefin) separator, and MCMB anode composite on copper. Cross-sectional scanning electron micrographs depict the microstructure for the (b) cathode and (c) anode, respectively [54].

In lithium ion battery cells, ions can be transported through the electrolyte upon electrochemical oxidation and reduction reaction, while electrons can simultaneously migrate through external wires connected to both electrodes. In anodes, electrochemical oxidation $\left(\mathrm{A} \rightarrow \mathrm{A}^{+}+\mathrm{e}^{-1}\right)$ occurs upon discharge, which is a conversion process from chemical energy to electric energy. In cathodes, electrochemical reduction $\left(\mathrm{B}^{+}+\mathrm{e}^{-1} \rightarrow \mathrm{B}\right)$ occurs as electrons are transported from the anode through an external circuit. An electrolyte is an ionic conductor to transport ions in between the anode and the cathode without conducing electrons. As seen by the schematic in Figure 3, the lithiated metal oxides and graphite carbons have layered structures 
with interstitial spaces accommodating lithium ions [51]. Such active materials are able to store the lithium ions by simultaneous electron transport from the current collector in order to reduce the lithium ion at the active material host site. The intercalation process can be carried out by a necessary solid-electrolyte-interface (SEI) that forms on the surface of electrodes, which plays a significant role in protecting the electrode surface from occurring further solvent reduction and act as a selective layer that conducts ions but nonconductive for electrons. The electrolyte formulation and origin of SEI components have been major research fields and there are current studies for new lithium salts and solvents for the improvement of ionic conductivity at various temperature ranges [55]. To date, there are three-major types of reaction mechanisms that are currently being investigated extensively for anode materials. to The mechanisms are briefly mention in the following section. 


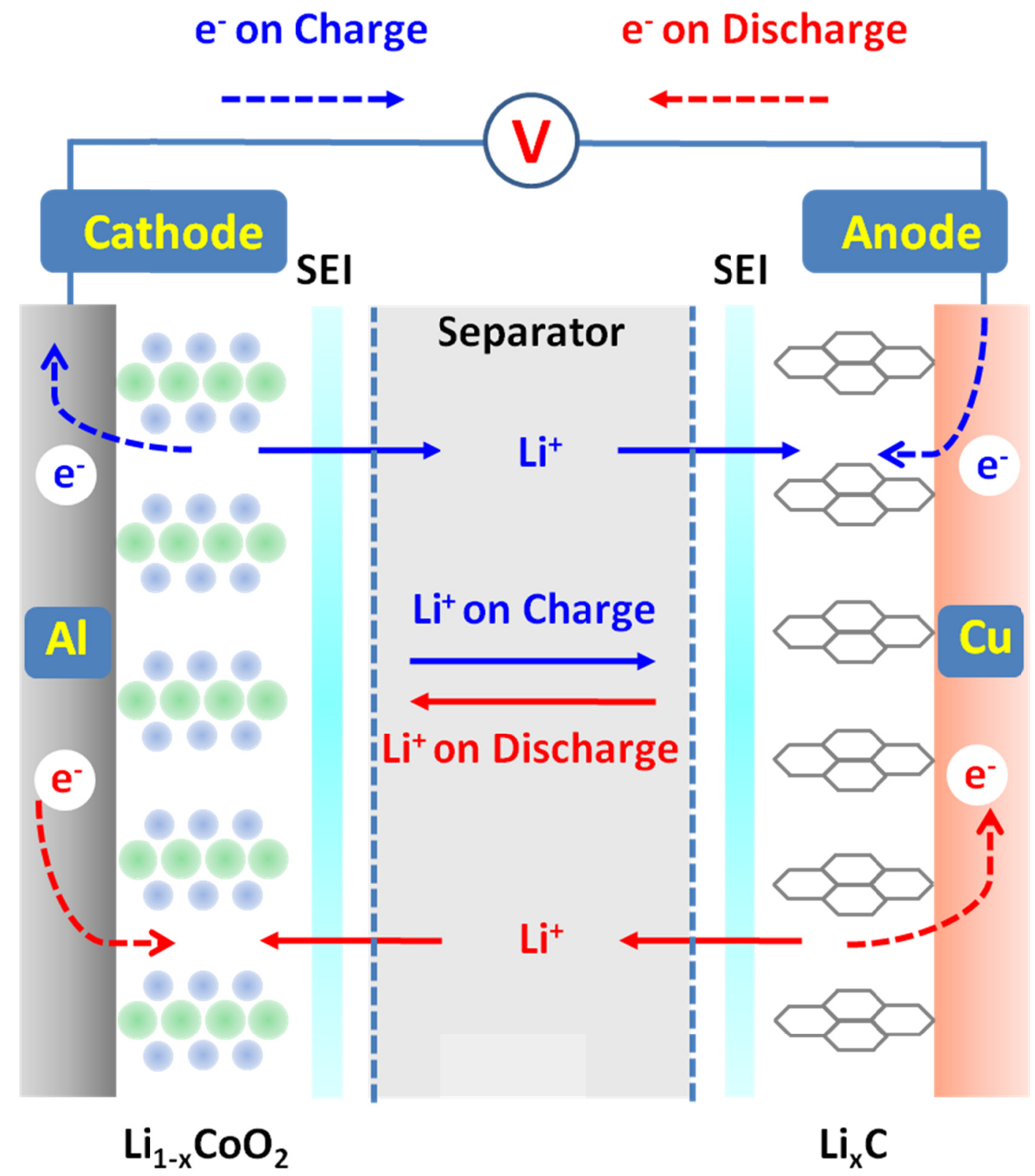

Figure 3. Schematic illustrating the operation mechanism of a lithium ion battery, including the movement of ions between electrodes (solid lines) and the electron transport through the complete electrical circuit (dashed lines) during charge (blue) and discharge (red) states. Figure 3 is adapted from reference [51]. 


\subsection{Major Types of Reaction Mechanism and Promising Active Materials for Anode}

\subsubsection{Formation Reactions in Reconstitution Reactions}

The equation for a formation reaction is expressed as:

$$
\mathrm{A}+\mathrm{B}=\mathrm{AB}
$$

in which a new phase $\mathrm{AB}$ is formed in one of the electrodes from its atomic constituents. The new phase $\mathrm{AB}$ can be created by moving one of the elements (e.g. A) from one electrode, passing across an electrochemical cell through the electrolyte, and subsequently reacting with the other component (e.g. B) in the other electrode. Since this formation reaction is accompanied by the microstructural variation in the new phase, the reaction is classified as a reconstitution reaction. For instance, lithium-silicon (Li-Si) and lithium-tin ( $\mathrm{Li}-\mathrm{Sn}$ ) alloys are formed by such reaction mechanism. The Li-Si alloy has, in its fully lithiated composition, $\mathrm{Li}_{4.4} \mathrm{Si}$, a theoretical specific capacity of 4,200 mAhg ${ }^{-1}$, which is known as the highest value, compared with 3,600 mAhg ${ }^{-1}$ for metallic Li and $372 \mathrm{mAhg}^{-1}$ for graphite. Unfortunately, the accommodation of so much lithium brings about enormous volume changes (300-400\%) during prolonged alloying and de-alloying processes between $\mathrm{Si}$ and $\mathrm{Li}$. The mechanical strain generated during the alloying and de-alloying processes leads to cracking and crumbling of the metal electrodes; as a consequence, a distinguishable loss of capacity to store charge appears in the course of a few cycles. To mitigate such pulverization effect, novel nanomaterials, such as Si nanotubes [15] and Si nanowires (Si NWs) [16], have been synthesized. C. K. Chan et al. recently demonstrated a silicon nanowirebased anode could almost constantly maintain the specific charge and discharge capacities higher than $3,000 \mathrm{mAhg}^{-1}$ for up to 10 cycles, as presented in Figure 4 [16]. 

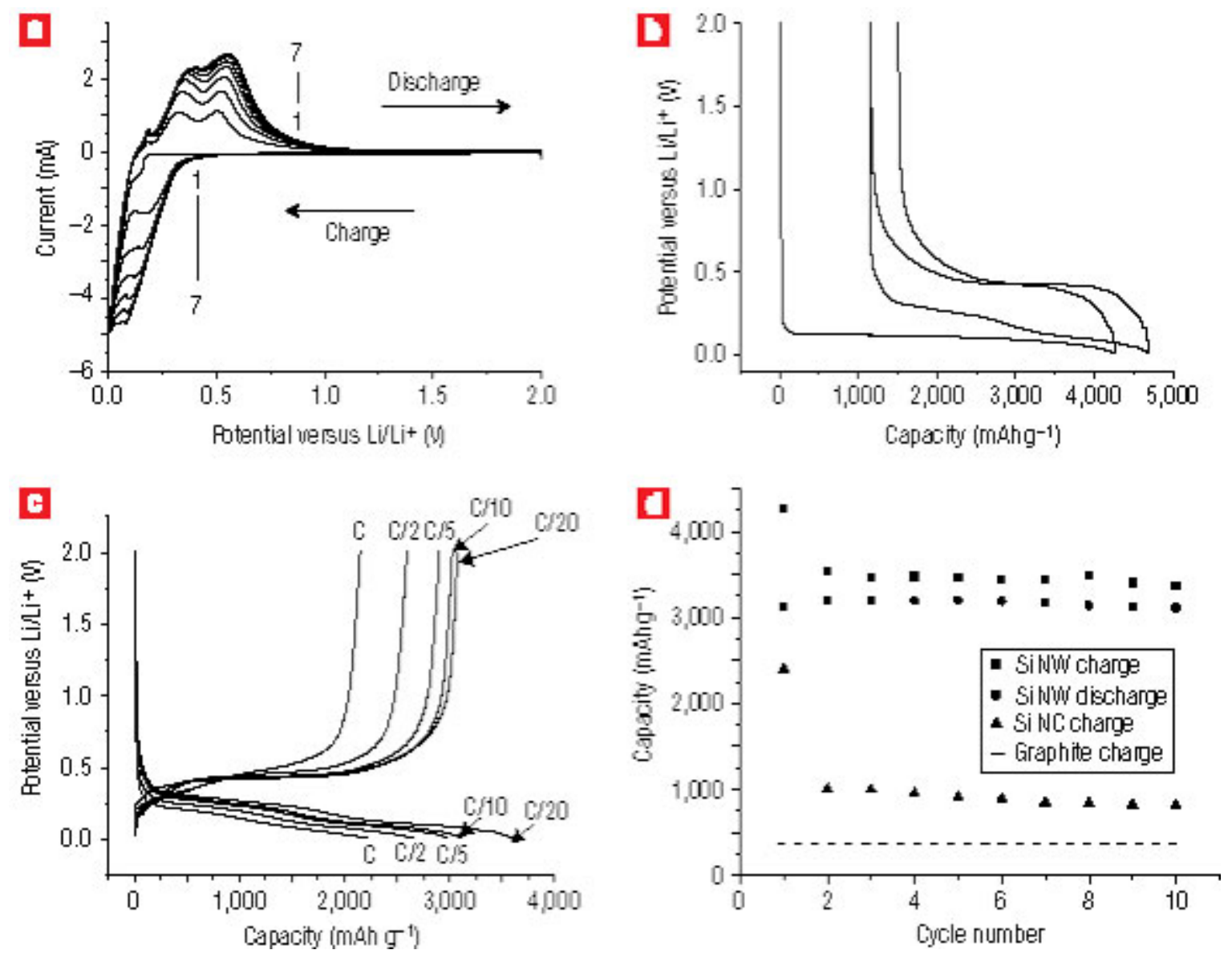

Figure 4. (a) Cyclic voltammogram for Si NWs from $2.0 \mathrm{~V}$ to $0.01 \mathrm{~V}$ versus $\mathrm{Li}^{\prime} \mathrm{Li}^{+}$at $1 \mathrm{mV} \mathrm{s}^{-1}$ scan rate. The first seven cycles are shown. (b) Voltage profiles for the first and second galvanostatic cycles of the Si NWs at the $\mathrm{C} / 20$ rate. The first charge achieved the theoretical capacity of 4,200 $\mathrm{mAhg}^{-1}$ for $\mathrm{Li}_{4.4} \mathrm{Si}$. (c) The voltage profiles for the Si NWs cycled at different power rates. The $\mathrm{C} / 20$ profile is from the second cycle. (d) Capacity versus cycle number for the Si NWs at the $\mathrm{C} / 20$ rate showing the charge (squares) and discharge capacity (circles). The charge data for Si nanocrystals (triangles) from reference [56] and the theoretical capacity for lithiated graphite (dashed line) are shown as a comparison to show the improvement when using Si NWs [16]. 


\subsubsection{Displacement Reaction in Reconstitution Reactions}

Another type of reconstitution reaction involves a displacement process, which can be simply written as:

$$
\mathrm{A}+\mathrm{BX}=\mathrm{AX}+\mathrm{B}
$$

where species A displaces species B in the binary phase BX, forming AX. Subsequently, a new phase accompanied by element $\mathrm{B}$ will be produced. A driving force creating this reaction can occur if phase AX has greater stability (i.e. the phase AX has a greater negative value of Gibbs free energy than that of phase BX). A good example is the redox reaction of transition metal oxide with Li metal. However, some transition metal oxides e.g. MO (M:Co, Ni, Fe etc.) have rock-salt structures; thus, there is no available empty space for insertion of lithium ions in the internal structure. Also, none of the $3 \mathrm{~d}$ transition metal can form an alloy with Li. The mechanism of such reaction differs from the classical reaction of $\mathrm{Li}$ insertion/deinsertion or Li-alloying processes and involves the formation and decomposition of $\mathrm{Li}_{2} \mathrm{O}$, accompanying the reduction and oxidation of metal nanoparticles (in the range of $1 \pm 5 \mathrm{~nm}$ ), respectively [10]. With smaller particle sizes of 5 and $10 \mathrm{~nm}$, more atoms lie near or on the surface of nanoparticles and occupy $15-30 \%$ and $30-60 \%$, respectively. Owing to the high surface energy of the metal nanoparticles, the formation of nanosize transition metal oxides e.g. $\mathrm{MO}\left(\mathrm{M}: \mathrm{Co}, \mathrm{Ni}, \mathrm{Fe}\right.$ etc.) from $\mathrm{Li}_{2} \mathrm{O}$ can occur. P. Poizot et al. proved the theory based on the fact that the electrochemical activity of nanoscale transition metal nanoparticles was more important and dependent on the degree of their division and agglomeration [18]. In the case of Co, the redox reactions can be expressed as:

$$
\begin{array}{r}
\mathrm{CoO}+2 \mathrm{Li}^{+}+2 \mathrm{e}^{-} \square \quad \mathrm{Li}_{2} \mathrm{O}+\mathrm{Co} \\
2 \mathrm{Li} \square \quad 2 \mathrm{Li}^{+}+2 \mathrm{e}^{-} \\
\mathrm{CoO}+2 \mathrm{Li} \square \quad \mathrm{Li}_{2} \mathrm{O}+\mathrm{Co}
\end{array}
$$


According to the redox reaction, one mole of Co reacts with one mole of lithium oxide to produce two moles of $\mathrm{Li}$ and one mole of cobalt oxide. As seen in Figure 5(a), the first discharge profile shows the characteristic rapid drop before reaching a plateau region (the potential and amplitude can be varied with the metal species) and then, decreasing to $0.01 \mathrm{~V}$. Figure 5(b) shows that the specific capacity keeps decreasing with the number of cycles, except in the case of CoO. Such a limitation has been generally shown in the electrochemical behavior of transition metal oxides due to the lower degree of division caused by the size of precursor particles, leading to further structural dismantlement of the metal particles upon cycling.
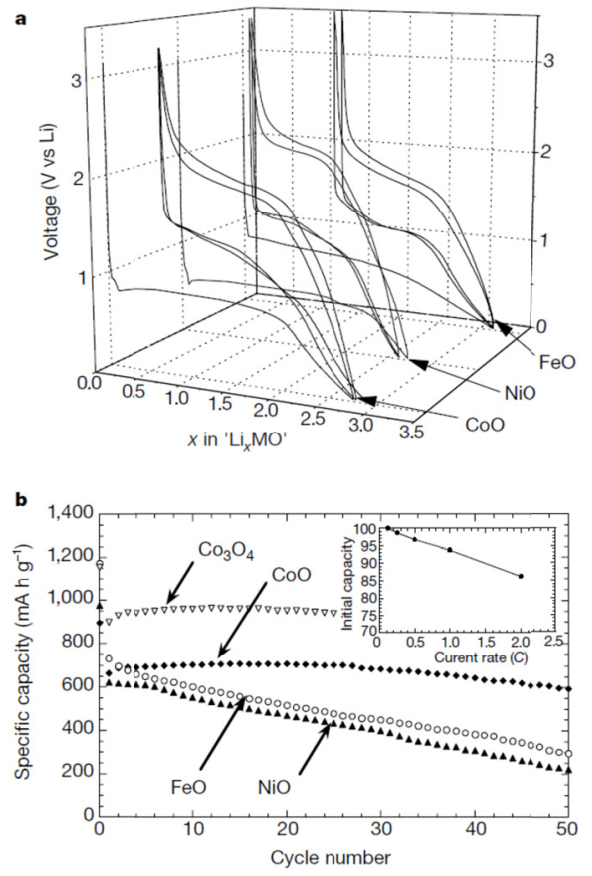

Figure 5. Properties of $\mathrm{MO} / \mathrm{Li}$ cells. (a) The voltage vs. composition profile for various $\mathrm{MO} / \mathrm{Li}$ cells cycled between $0.01 \mathrm{~V}$ and $3 \mathrm{~V}$ at a rate of $\mathrm{C} / 5$ (1 lithium in 5 hours). The capacity fading for the same cells under similar conditions is shown in (b), where we also show data for a $\mathrm{Co}_{3} \mathrm{O}_{4} / \mathrm{Li}$ cell in order to show that the reported behavior is not specific to divalent oxides. Inset, the rate capability of a $\mathrm{CoO}$ electrode [18]. 


\subsubsection{Insertion Reactions}

This involves the insertion of guest species into normally unoccupied interstitial sites in the crystal structure of an existing stable host material. In the insertion reaction, the chemical composition of the host phase initially present can be substantially changed; however, this reaction theoretically does not result in a change in its basic crystal structure or the number of the phases in the microstructure. On the other hand, the insertion and extraction of interstitial species into and from previous unoccupied locations in the structure generally result in a change in volume. Such volume change induces mechanical stress and energy in the structure, which plays a significant role in the hysteresis and subsequent energy loss, observed in a number of reversible battery electrode reactions. Regarding the intercalation and decalation reaction with $\mathrm{Li}$, a lithiumgraphite compound can be chosen as a good example. Graphitic carbon fundamentally comprising $\mathrm{sp}^{2}$-hybridized carbon atoms, which are arranged in a planar "honeycomb-like" network, i.e., a "graphene" layer is formed (see Figure 6) [57]. Van der Waals forces provide a weak cohesion of the graphene layers, resulting in the layered graphite structure. From a strictly crystallographic point of view, the term "graphite" is only used for carbons with a perfect stacking order of graphene layers, either the predominant $\mathrm{AB}$ (hexagonal graphite, $2 \mathrm{H}-$, or $\alpha-$ phase) or the less common ABC (rhombohedral graphite, 3R-, or $\beta$-phase). Due to the small

energy for transformation of the $\mathrm{AB}$ into the $\mathrm{ABC}$ type of stacking (and vice versa), perfectly stacked graphite crystals are not readily available. Therefore, the term "graphite" is often used regardless of stacking order. 


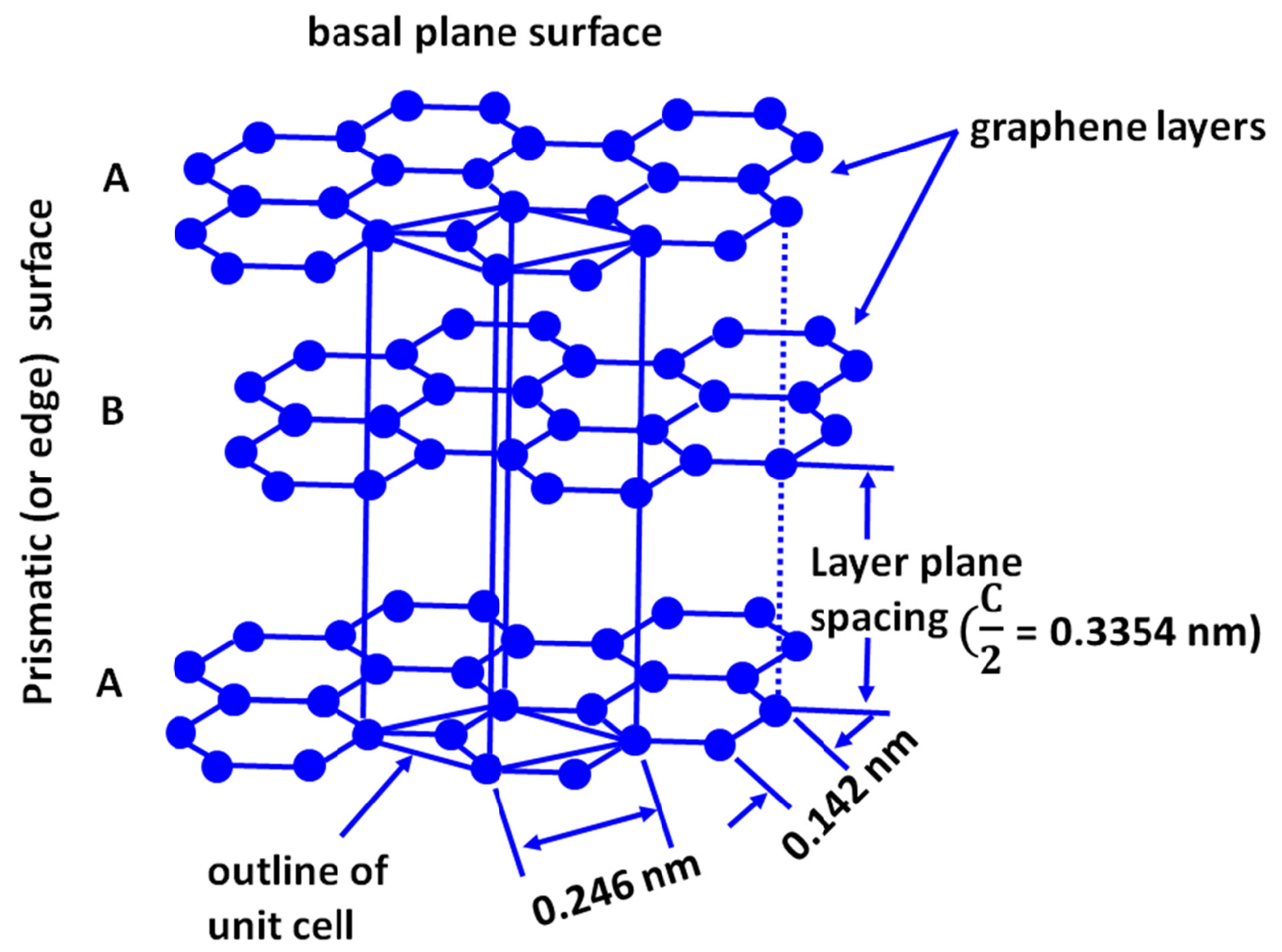

Figure 6. Crystal structure of hexagonal graphite showing the AB layer stacking sequence, the unit cell, and the subdivision in basal plane and prismatic surfaces [57].

The ratio of hexagonal to rhombohedral structures can be varied by certain processes. Mechanical treatments, especially milling, shearing, or ultrasonic impact result in a larger extent of the $\beta$-phase, whereas thermal annealing procedures at elevated temperatures transform the material to the thermodynamically more stable $\alpha$-form. By heat treatment, it is possible to create a graphite which contains $100 \% \alpha$-phase, whereas this could not be achieved by mechanical measures for the $\beta$-form. So far, $\beta$-phase contents up to $30 \%$ have been reported. The rhombohedral and hexagonal contents of graphites can be identified from XRD spectra [58]. 
It should be noted here, that in addition to a change of the stacking sequence, mechanical milling can destroy carbon-carbon bonds and thus, increase the number of structural defects. The kind and extent of defects are also dependent on the milling procedure. On the contrary, heattreatment can increase the degree of crystallinity in graphitic structure by reducing the number of defects. Shorter milling time can confine the amorphization to the surface. Milling can also change the chemical (surface) composition of the carbon material under certain conditions since the defects created by milling are highly reactive.

Layered graphite displays basically two kinds of structural surfaces which are prismatic (edge) and basal plane (Figure 6). Ideal (i.e. defect- and contaminant-free) basal plane surfaces are homogeneous and smooth and consist of carbon atoms. In contrast, the prismatic surfaces are heterogeneous and rough and apart from carbon may contain various, mostly (but not only) oxygen-containing, surface groups. The quantity and quality of surface groups for graphitic and non-graphitic layers can be modified by mechanical (e.g., milling), thermal (e.g., heat treatment), and chemical measures (e.g., exposure to reagents creating a certain surface chemistry), or a combination of these methods. It is known that intercalation of $\mathrm{Li}^{+}$ions into the graphitic carbon proceeds via the prismatic planes and not via the basal planes. The interaction of graphite with the electrolyte is also dependent on the respective surface and its properties. The major advantage of graphitic materials is volume expansion of around $12 \%$ which is presumably due to their unique mechanism of intercalation and decalation of $\mathrm{Li}^{+}$ions, as shown in Table II [59]. Also, as shown in Figure 7, the battery voltage is determined by the electrochemical potential difference between the cathode and anode with respect to lithium metal [51-52, 60-61]. Additionally, the open circuit voltage of graphite ranges between 0.1 and $0.2 \mathrm{~V}$ vs. $\mathrm{Li} / \mathrm{Li}^{+}$which is the lowest value compared with any other anode material. Owing to those features, graphite has been utilized as the anode for commercially available batteries. However, the lower specific capacity of $372 \mathrm{mAhg}^{-1}$ of graphite is insufficient for next-generation electric vehicles which demand higher capacity. To 
address this issue, MWCNTs have been proposed as promising anode materials to open up a new avenue for high-performance electric vehicles and electronic portable devices.

Table II. Capacities and volume changes of different elements [59]

\begin{tabular}{|l|c|c|c|c|c|}
\hline Starting material & C & Al & Si & Sn & Bi \\
\hline Lithiated phase & LiC6 & LigAl4 & Li21Si5 & Li17Sn4 & Li3Bi \\
\hline $\begin{array}{l}\text { Theoretical specific } \\
\text { capacity (Ah/kg) }\end{array}$ & 372 & 2235 & 4010 & 959 & 385 \\
\hline $\begin{array}{c}\text { Theoretical volumetric } \\
\text { capacity (Ah/I) }\end{array}$ & 833 & 6035 & 9340 & 7000 & 3773 \\
\hline \begin{tabular}{c} 
Volume change (\%) \\
\hline
\end{tabular} & 12 & 238 & 297 & 257 & 115 \\
\hline
\end{tabular}

\subsection{Brief Introduction to Carbon Nanotubes}

Carbon nanotubes can be envisioned as a rolled up graphene sheet into a seamless cylinder with fullerene caps for single wall (SWCNTs) or multiple sheets (MWCNTs). Characteristics of MWCNTs are the concentric layers that are spaced $0.34 \mathrm{~nm}$ apart as illustrated in the transmission electron micrograph (TEM of Figure 8(a)-(c). In the case of SWCNTs, there are van der Waals interactions between sidewalls that lead to hexagonally close-packed bundles, as shown in Figure 8(d) [62]. The electronic properties of SWCNTs are determined by the chirality of the SWCNT, which represents the diameter and helicity of the structure. The chirality determines whether the nanotubes will be metallic or semiconducting in nature. Figure 9 demonstrates the basics for assigning SWCNT chiralities with a roll-up vector $\left(n \mathrm{a}_{1}+m \mathrm{a}_{2}\right)$ from a point of origin on a graphene sheet. The chiral angle $\left(\leq 30^{\circ}\right)$ is the angle between the zigzag axis 
and the roll-up vector [54]. The result from this exercise is a series of chiral indices with integer values of $n$ and $m$, which are used to assign the uniqueness of each SWCNT.

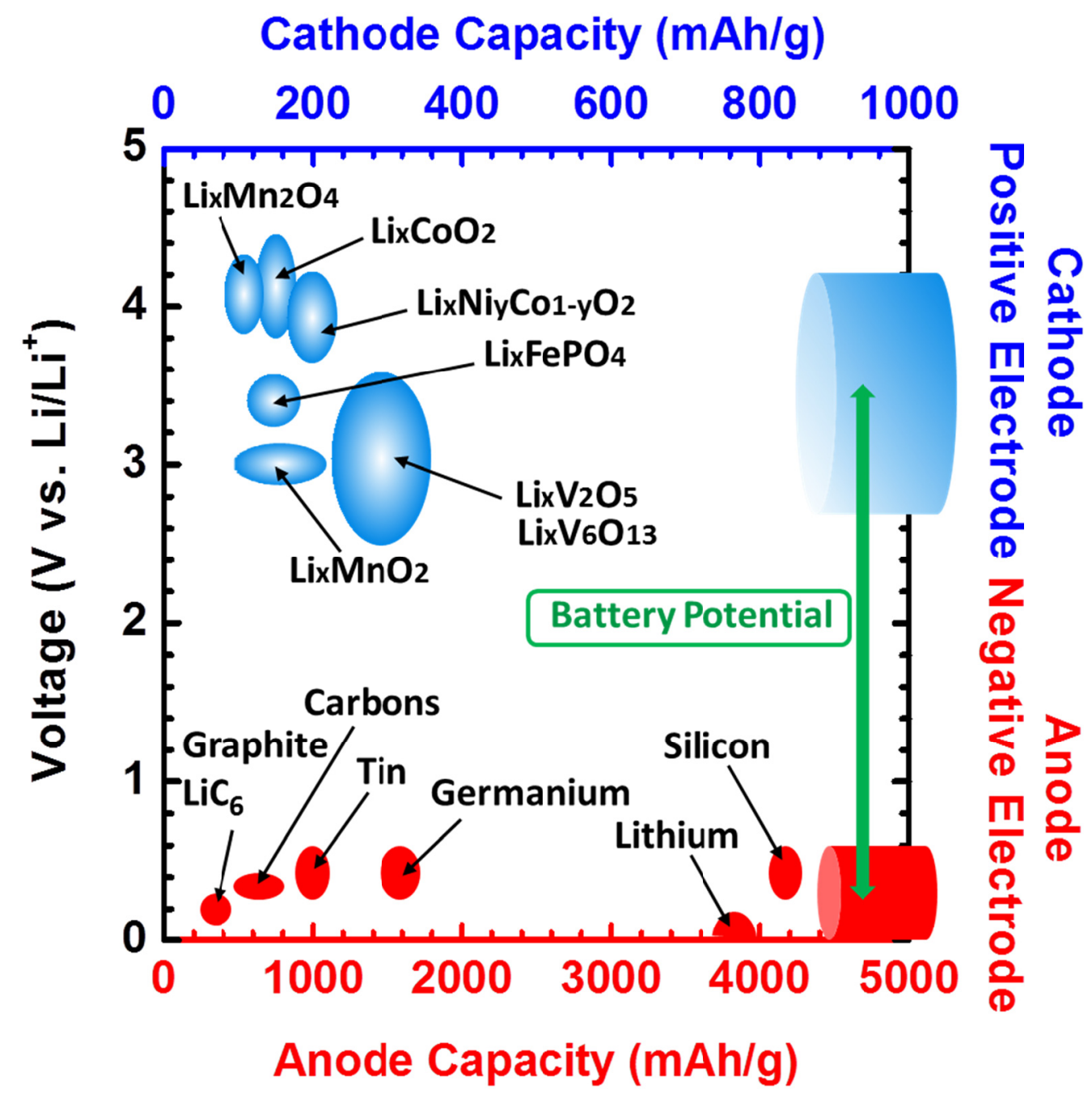

Figure 7. Diagram illustrating the lithium ion capacity and electrochemical reduction potentials with respect to lithium metal for conventional anode (red axis) and cathode materials (blue axis). The battery potential is the relative difference between the voltage of the selected positive electrode materials (blue ovals) and voltage of the corresponding negative electrode material (red ovals). Figure data are adapted from references [51-52, 60-61]. 

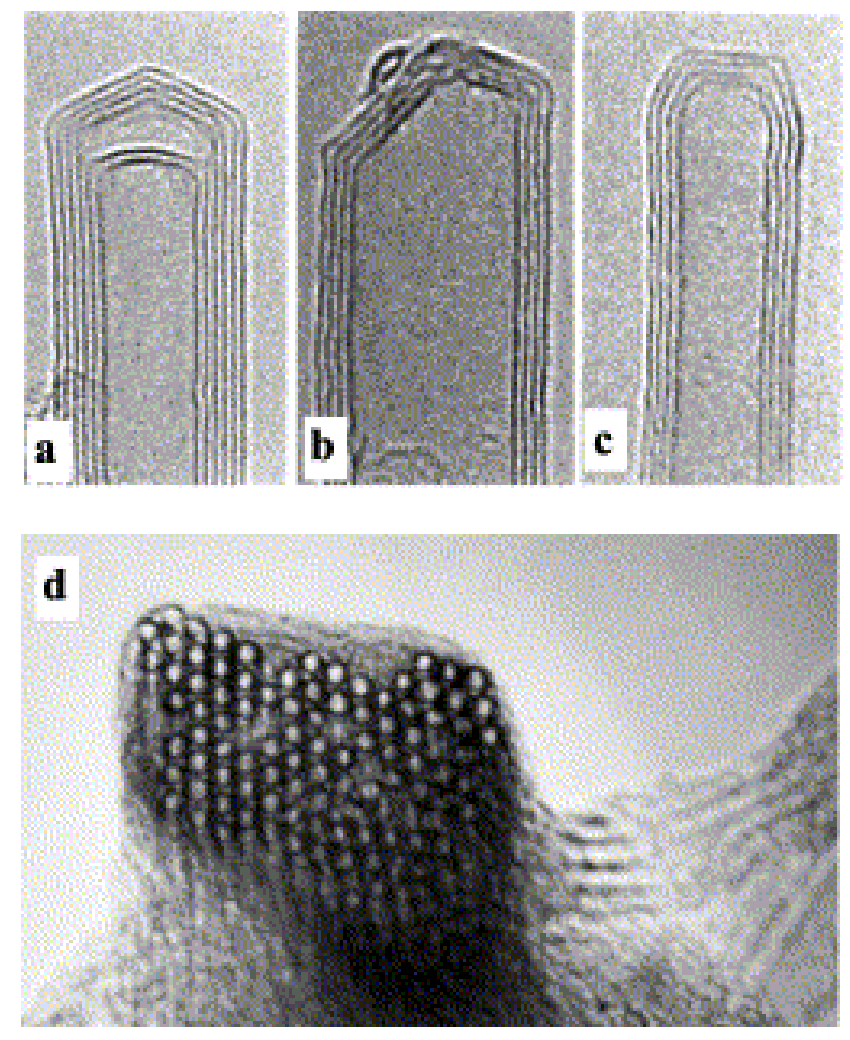

Figure 8. Carbon nanotubes imaged by transmission electron microscopy (TEM). TEM is a technique used in the discovery of both MWCNTs and SWCNTs. (a-c) TEM images of MWCNTs with closed caps (courtesy of S. Iijima). The parallel lines are the cross-sections of the sidewalls of concentric cylinders. Diameters of MWCNTs are typically on the order of 10-20 nm. (d) TEM image of the cross-section of a bundle of SWCNTs (courtesy of R. Smalley). Each circle represents the cross-section of a SWCNT with diameter $\sim 1.4 \mathrm{~nm}[62]$. 


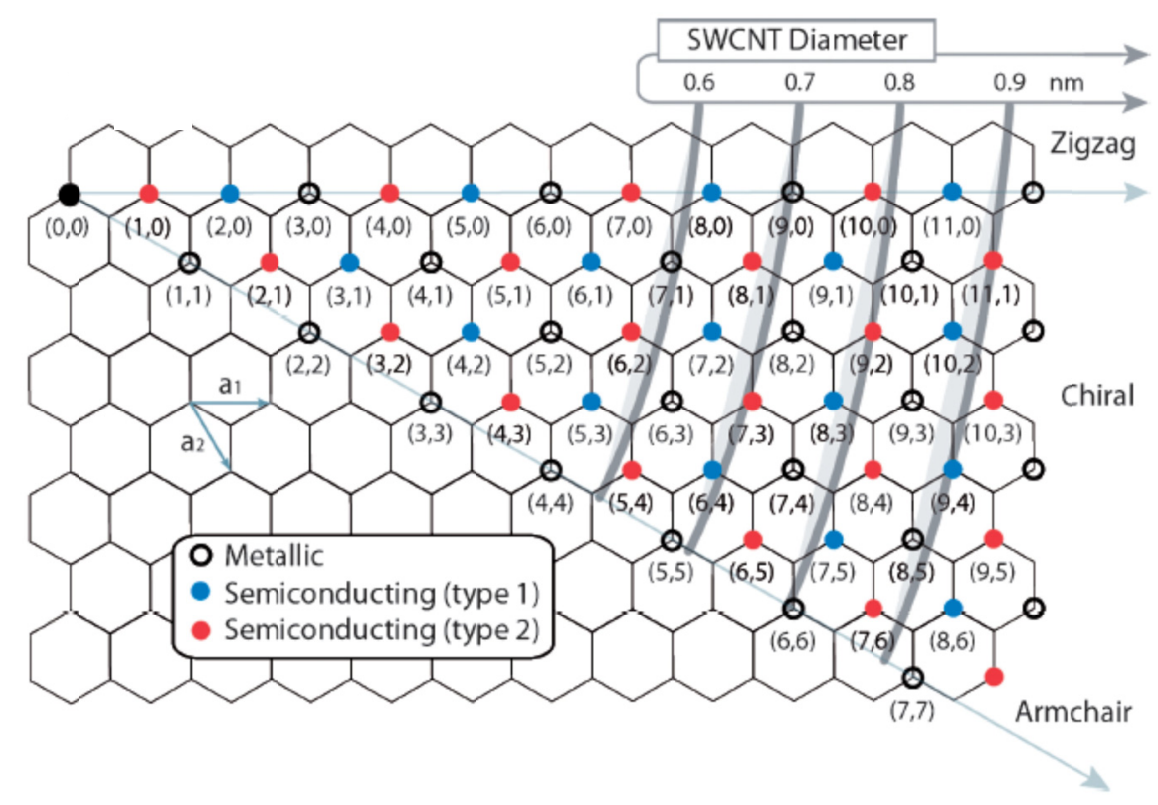

Figure 9. Chirality chart illustrating the assignment of $(n, m)$ SWCNT structures using a role-up vector from an origin point on a graphene sheet and the nanotube diameter [54].

There has been intensive effort to develop control over individual CNT properties (chirality, length, purity, electronic type ratio, etc.) for a variety of applications. This control can either be accomplished during synthesis or in conjunction with followed chemical and physical treatments aimed at purification and separation of desired products. The most common techniques are chemical vapor deposition (CVD), arc-discharge, and laser vaporization synthesis. In this dissertation, the brief introduction to CVD method will be presented in the Chapter 3 .

\subsection{Carbon Nanotubes for Li-ion Battery Anode}

MWCNTs are allotropes of carbon constructed with their one dimensionally long and cocentric hollow structure with the walls formed by one-atomic thick sheet of carbon, called graphene [63]. MWCNTs have been considered one of the strong candidates for anode materials for high-performance LIB, which is associated with their unique multi-walled co-centric tube 
geometry, excellent electrical properties, wide electrochemical-stability window and highly accessible surface area [64]. Carbon nanotubes contain many kinds of the interstitial sites (intershell van der Waals spaces, inter-tube channels, and inner cores), which are accessible for Li intercalation [64]. Therefore, it has been speculated that a higher Li capacity may be obtained in carbon nanotubes. Electrochemical intercalation of MWCNTs [26-27] and SWCNTs [32] has been investigated by several groups. Figure 10 presents a galvanostatic charge and discharge profile that is typical for all the family of carbon nanomaterials, including SWCNTs, MWCNTs and carbon nanofibers [65]. A reversible capacity $\left(\mathrm{C}_{\mathrm{rev}}\right)$ of $100-640 \mathrm{mAhg}^{-1}$ has been reported, depending on the sample processing and annealing conditions of MWCNTs [26-27] and SWCNTs [32].

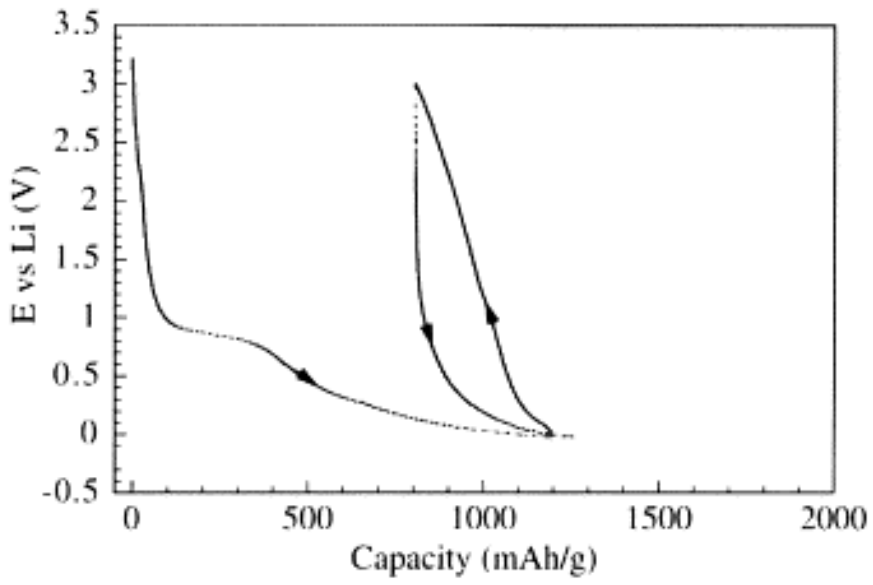

Figure 10. Galvanostatic insertion-extraction of lithium into and from carbon nanotubes. Current load of $20 \mathrm{mAg}^{-1}[65]$.

In general, well-graphitized MWCNTs synthesized by an arc-discharge method have a lower reversible specific capacity $\left(\mathrm{C}_{\mathrm{rev}}\right)$, compared with the value obtained from defective MWCNTs 
prepared by a CVD method. From structural studies, it has been proven that alkali metals can be intercalated into the inner-shell spaces within the individual MWCNTs through defect sites [66]. However, one of the most critical disadvantages of the CNT-based materials is the large irreversible specific capacity during a primary cycle. The main reasons for such a problem are strongly related to the large surface area of a CNT-based electrode (e.g. $\sim 300 \mathrm{~m}^{2} \mathrm{~g}^{-1}$ for SWCNT films measured by the Branuer-Emmett Teller (BET) method) and the formation of solid electrolyte interface (SEI) layers on the surface of the CNT-based electrodes. It should be highlighted that the research into CNT-based anode materials has been mainly performed through their composite form combined with binder and conductive additive. Consequently, there have been some drawbacks, such as harmful effect of the polymeric binder and extra weight of the active material.

To avoid such limitation, Dr. Won Bong Choi's group at FIU has recently developed engineered carbon nanotube-based system, specifically, direct MWCNT grown on $\mathrm{Cu}$ substrates by catalytic thermal CVD and demonstrated excellent properties for application in LIB electrodes [34]. An MWCNT grown on $\mathrm{Cu}$ anode showed very high specific capacity (for the second discharge cycle, it was $\left.1,455 \mathrm{mAhg}^{-1}\right)$ and exceptional stability of the reversible capacity $(\sim 900$ $\mathrm{mAhg}^{-1}$ ) at $1 \mathrm{C}$ rate, as presented in Figure 11. In addition, the anode system has major advantages of binder-free so that the LIB button cell composed of the anode can avoid any severe safety concern. It is known that the most widely used binder, poly (vinylidene fluoride) (PVdF), can react with electrolyte. Moreover, $\mathrm{PVdF}$ is known to react with graphitic materials and metallic lithium to form stable compounds [67-68]. Such reactions are highly exothermic; for example, the reaction with metallic Li produces $7.2 \mathrm{~kJ}$ of energy per gram of PVdF [67]. Thus, the presence of a binder could lead to thermal runaway, necessitating incorporation of additional safety features in the battery. 

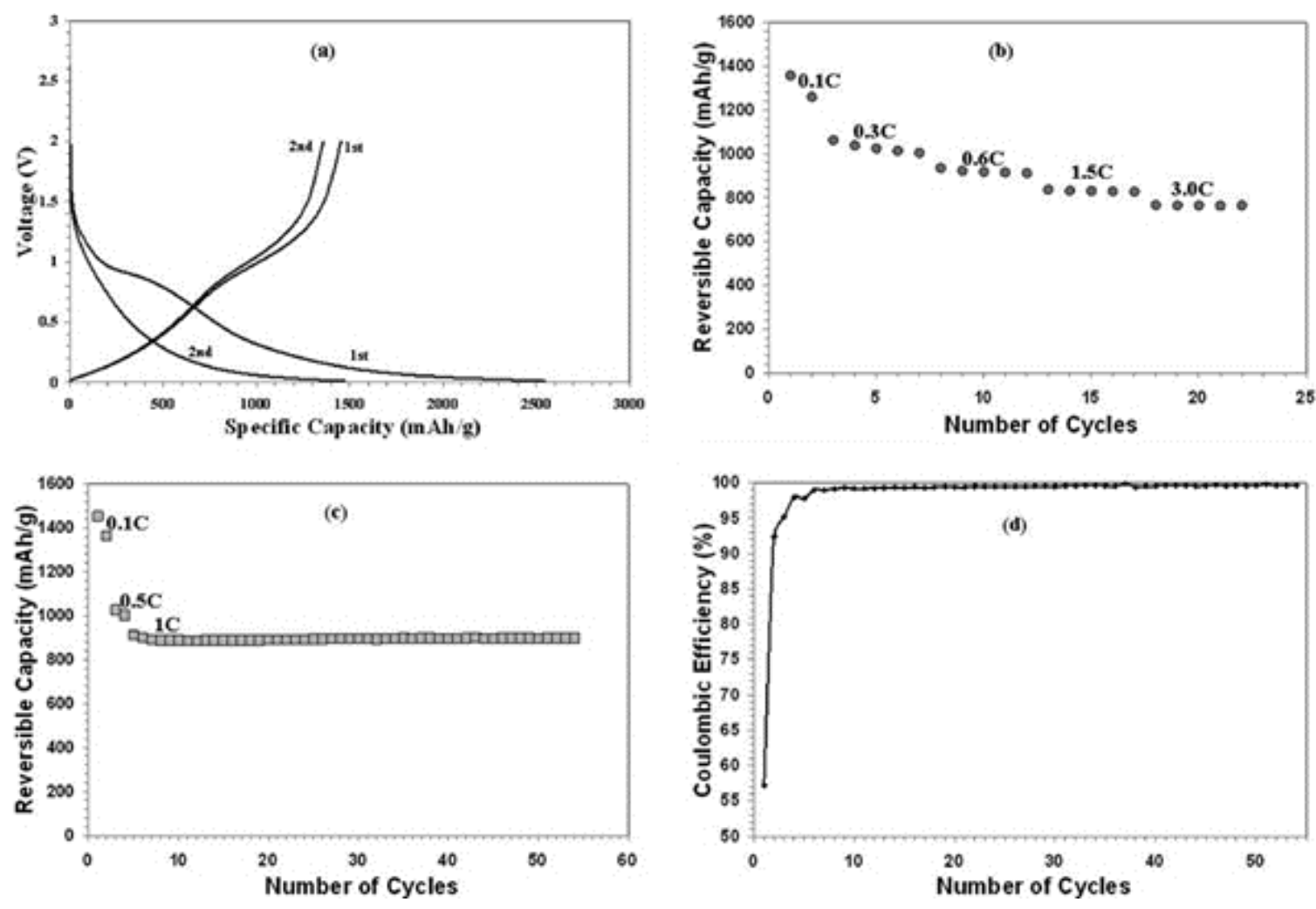

Figure 11. Electrochemical characteristics of the proposed CNT-based electrode structure. (a)

First two charge-discharge cycles of the MWNT-on-Cu anode, at $0.1 \mathrm{C}$ rate. The first discharge cycle (lithiation) has shown a very high capacity of $2,547 \mathrm{mAhg}^{-1}$, while the following delithiation cycle showed a specific capacity of $1,455 \mathrm{mAhg}^{-1}$. (b) Reversible capacity of the MWNT-on-Cu anode, at different C-rates. Very high specific capacity could be observed at all Crates. Even at the $3 \mathrm{C}$ rate, a reversible capacity of $767 \mathrm{mAhg}^{-1}$ was observed, which is almost 2 times the theoretical capacity of graphite. (c) Exceptional stability of the reversible capacity $\left(\sim 900 \mathrm{mAhg}^{-1}\right)$ of the MWNT-on-Cu anode in long-run, at $1 \mathrm{C}$ rate. Except for the first two cycles, virtually no capacity degradation was observed for this anode structure, in 50 cycles. (d) Coulombic efficiency of the MWNT-on-Cu anode, showing very high efficiency, except for the first cycle. After the initial five cycles, the efficiency remained more than $99 \%$ [34]. 
In the case of the directly grown CNTs on the current collector, several features of the novel anode system can be summarized. First, it reduces harmful effect of the polymeric binder and weight of the active material, increases specific capacity, and shows potential to be used for hightemperature application. Second, CNTs do not have any kind of expansion/contraction and pulverization problems (like $\mathrm{Si}$ and $\mathrm{SnO}_{2}$ ), so they can sustain their capacity for long cycles. Third, each CNT is well bonded to the current collector, thus all of them contribute to the capacity. Fourth, the high specific surface area of CNTs allows more Li ion intercalation. Fifth, higher conductivity of the active anode material is important for achieving higher capacity. In that respect, MWCNTs, known to be excellent charge carriers, are an alternative option and aid in achieving higher capacity. Moreover, by the interface-control proposed in this study, an ohmic contact and strong bonding between the CNTs and substrate are ensured, which further helps in efficient charge transport [69]. Furthermore, the anode structure, consisting of MWCNTs grown on $\mathrm{Cu}$ foil, can be very easily fabricated using the thermal chemical vapor deposition (CVD) process. The listed advantageous features of the CNT directly grown on metal substrate can be schematically illustrated, as presented in Figure 12.

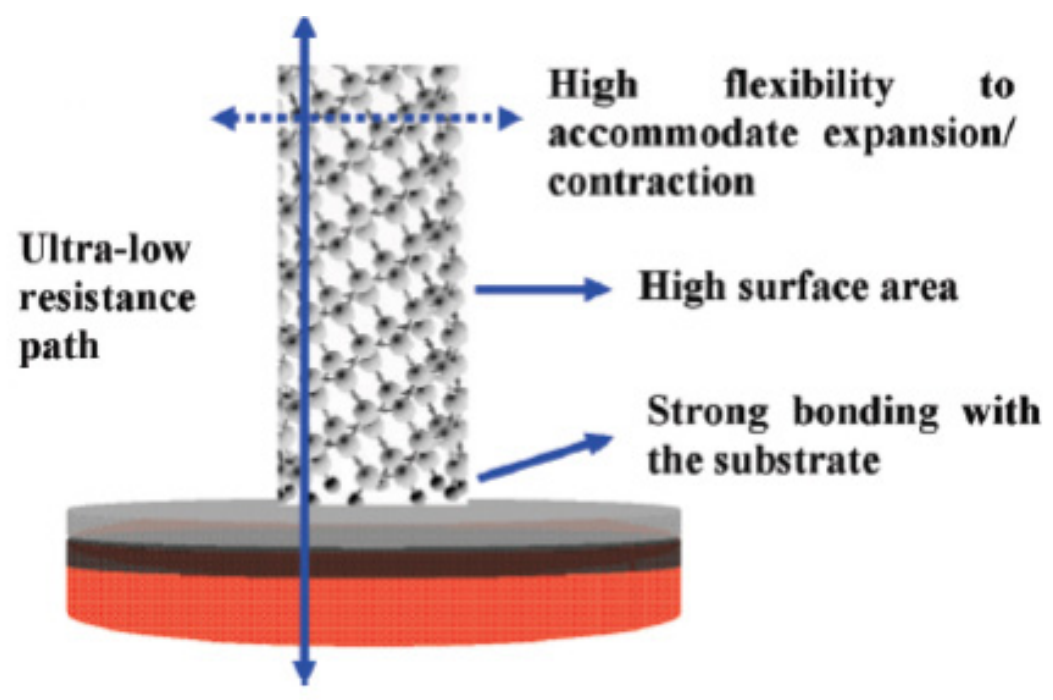

Figure 12. A schematic (not to scale) of the proposed anode structure, showing its advantageous features [34]. 


\subsection{Hybrid Anode Structures for High-Performance Li-ion Batteries}

Recently, in order to obtain better performance, many research groups are interested in the active/inactive nanocomposite concept to be applied to advanced Li ion battery anode system. Several authors have proved that this approach is effective to synergize Li-ion battery performance through intimately mixing two materials, one reacting with lithium whereas the other acting as an inactive mechanical support to avoid pulverization of the anode structure upon $\mathrm{Li}^{+}$cycling [41, 60]. L. F. Cui et al. synthesized a CNT-Si composite film [41]. CNTs are infiltrated in a Si film and play an important role in improving a structural reinforcement and electric conductivity in the composite anode. Figure 13(b) illustrates the free-standing CNT-Si hybrid structure and it can be rippled up to release the large strain of $\mathrm{Si}$ induced during $\mathrm{Li}^{+}$ion charge and discharge cycling. By this method, the relative thickness up to $4 \mu \mathrm{m}$ of Si film demonstrates a good cycling performance.

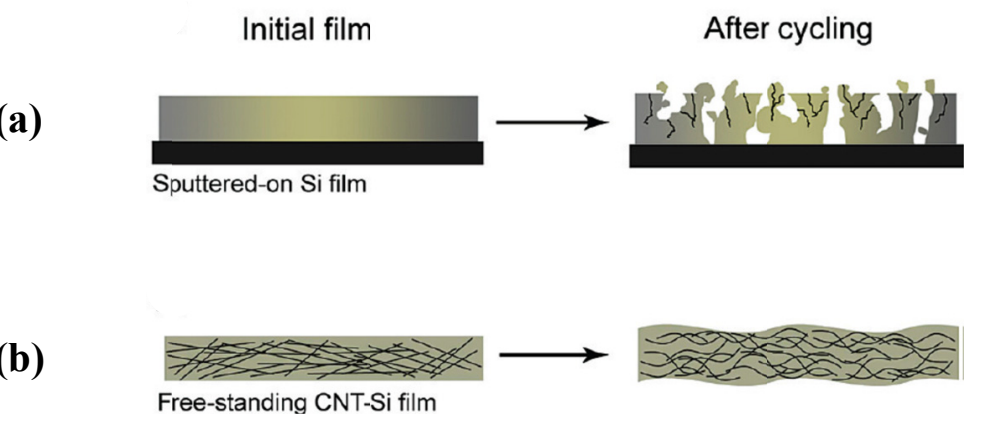

Figure 13. (a) Sputtered-on Si film tends to pulverize after $\mathrm{Li}^{+}$cycling. (b) CNT-Si films can "ripple up" to relax the large strain during $\mathrm{Li}^{+}$cycling [41]. 
A photograph of a Si-free-standing CNT composite anode is presented in Figure 14(a). Also, Figure 14(b) shows the cycling performance of the hybrid anode. Discharge specific capacity remains $1,711 \mathrm{mAhg}^{-1}$ (or $82 \%$ with respect to the initial value) after 50 cycles. The Coulombic efficiency is initially $86 \%$ and greater than $98 \%$ throughout the rest of cycles.

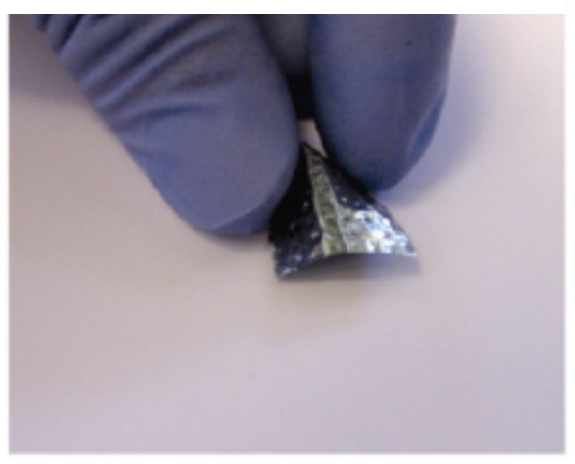

(a)

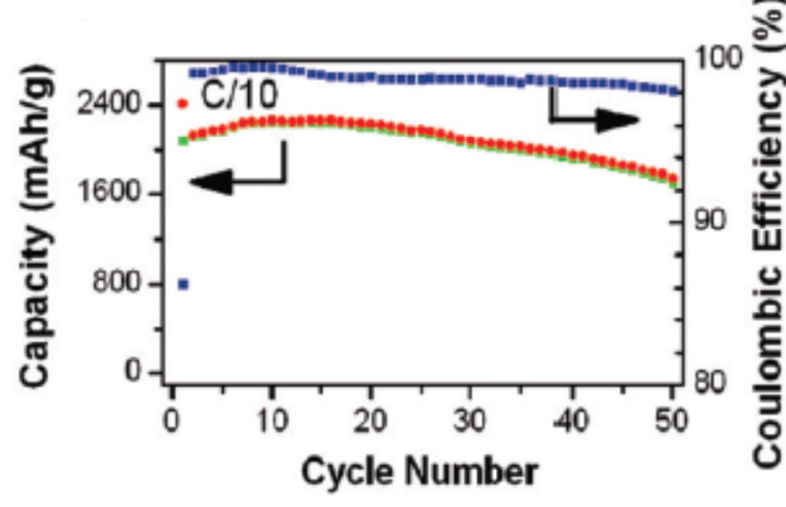

(b)

Figure 14. (a) Photograph of a free-standing CNT-Si film. (b) Charge (red) and discharge (green) capacity and Coulombic efficiency (blue) versus cycle number for a half cell using free-standing CNT-Si films as the working electrode cycled between $1-0.01 \mathrm{~V}$ [41].

\subsection{D Anode Architecture for High Loading Density of Active Materials}

Very recently, it is argued that nanotube-based active materials have a critical shortcoming in terms of their very low weight per unit electrode area [36]. Thus, their gravimetric energy density may not give a realistic picture to commercial application. The critical limitation may lead to scale-up issues for their potential application in the development of EV. 

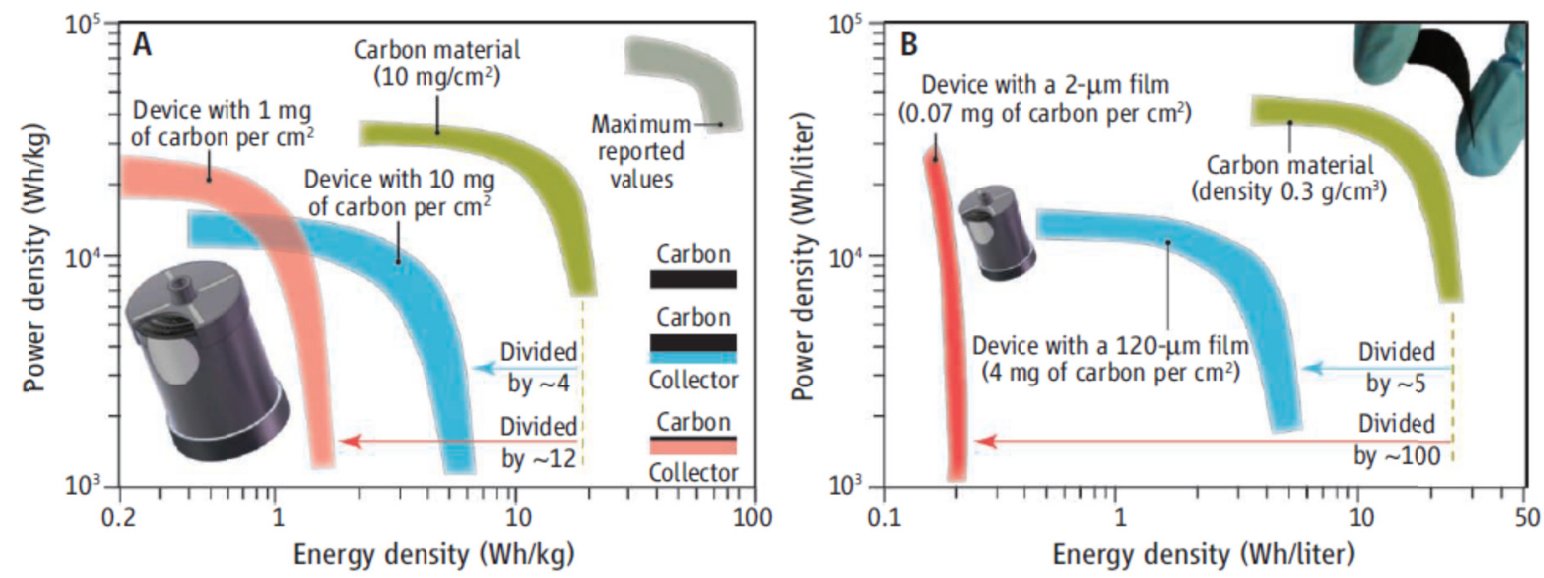

Figure 15. One way to compare electrical energy storage devices is to use Ragone plots, which show both power density (speed of charge and discharge) and energy density (storage capacity). These plots for the same electrochemical capacitors are on a gravimetric (per weight) basis in (A) and on a volumetric basis in (B). The plots show that excellent properties of carbon materials will not translate to medium- and large-scale devices if thin-film and/or low-density electrodes are used [36].

To address this issue, we would like to propose a new geometry of 3D $\mathrm{Cu}$ current collectors, which can create higher surface area to accommodate higher solid loading of MWCNTs on the uniformly arrayed patterns in the $3 \mathrm{D}$ structure, leading to higher specific capacity and C-rate capability. To date, intensive research has been performed to improve Li-ion battery properties through introducing a number of 3D structured current collectors, including carbon papers [37], a self-assembled 3D bicontinuous nanoarchitecture [38], aluminum nanorods [39], and nanoporous nickel [40]. In the previous research, it was proved that a self-assembled 3D bicontinuous nanoarchitecture could be one of the ideal electrode architectures in order to realize not only high volume fraction of nanostructured electrolytically active materials $\left(\mathrm{NiOOH} / \mathrm{Nickel}\right.$ and $\mathrm{MnO}_{2}$ 
cathodes) but also their efficient ion and electron transport [38]. Figure 16 shows the schematic diagram of bicontinuous electrode fabrication process. In addition, ALD coated $\mathrm{TiO}_{2}$ anodes on 3D aluminum nanorod current collectors showed a ten-time increase in their theoretical area and total capacity $\left(0.0112 \mathrm{mAhcm}^{-2}\right)$, compared to those resulted from the same anodes on 2D flat aluminum plate and high rate capability (The capacity ratios at $10 \mathrm{C} / 0.5 \mathrm{C}$ and $20 \mathrm{C} / 0.5 \mathrm{C}$ of the 3D anode were 0.4 and 0.35 , respectively.) [39].

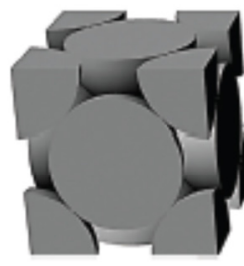

Opal template

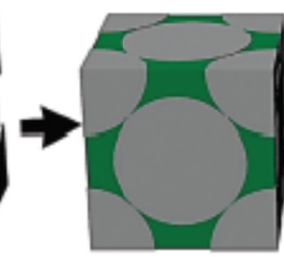

Electrodeposit nickel

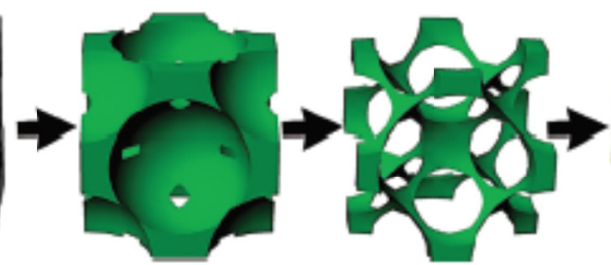

Remove template

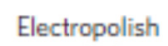

nickel

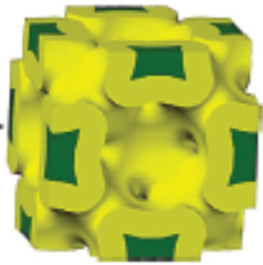

Electrodeposit active materials

Figure 16. Bicontinuous electrode fabrication process. The electrolytically active phase is yellow and the porous metal current collector is green. The electrolyte fills the remaining pores [38].

A prototype Li-ion battery was assembled from a bicontinuous lithiated $\mathrm{MnO}_{2}$ cathode, together with a graphite anode, a separator and $1 \mathrm{M} \mathrm{LiClO}_{4}$ as the electrolyte, and sealed in a moisture barrier bag [38]. The anode capacity was selected to be about ten times greater than that of the cathode so that the anode was not rate-limiting. A constant potential (3.6 V) was applied. The initial charge current was $35 \mathrm{Ag}^{-1}(\sim 177 \mathrm{C})$ and rapidly decreased to almost zero in $120 \mathrm{~s}$ (see Figure 17). The discharge curves in Figure 17 show that after $60 \mathrm{~s}$, the battery is charged to $75 \%$ capacity, and after $120 \mathrm{~s}$, it is $90 \%$ charged relative to a $800 \mathrm{~s}$ charge. Charging past $800 \mathrm{~s}$ resulted in insignificant increases in capacity. 


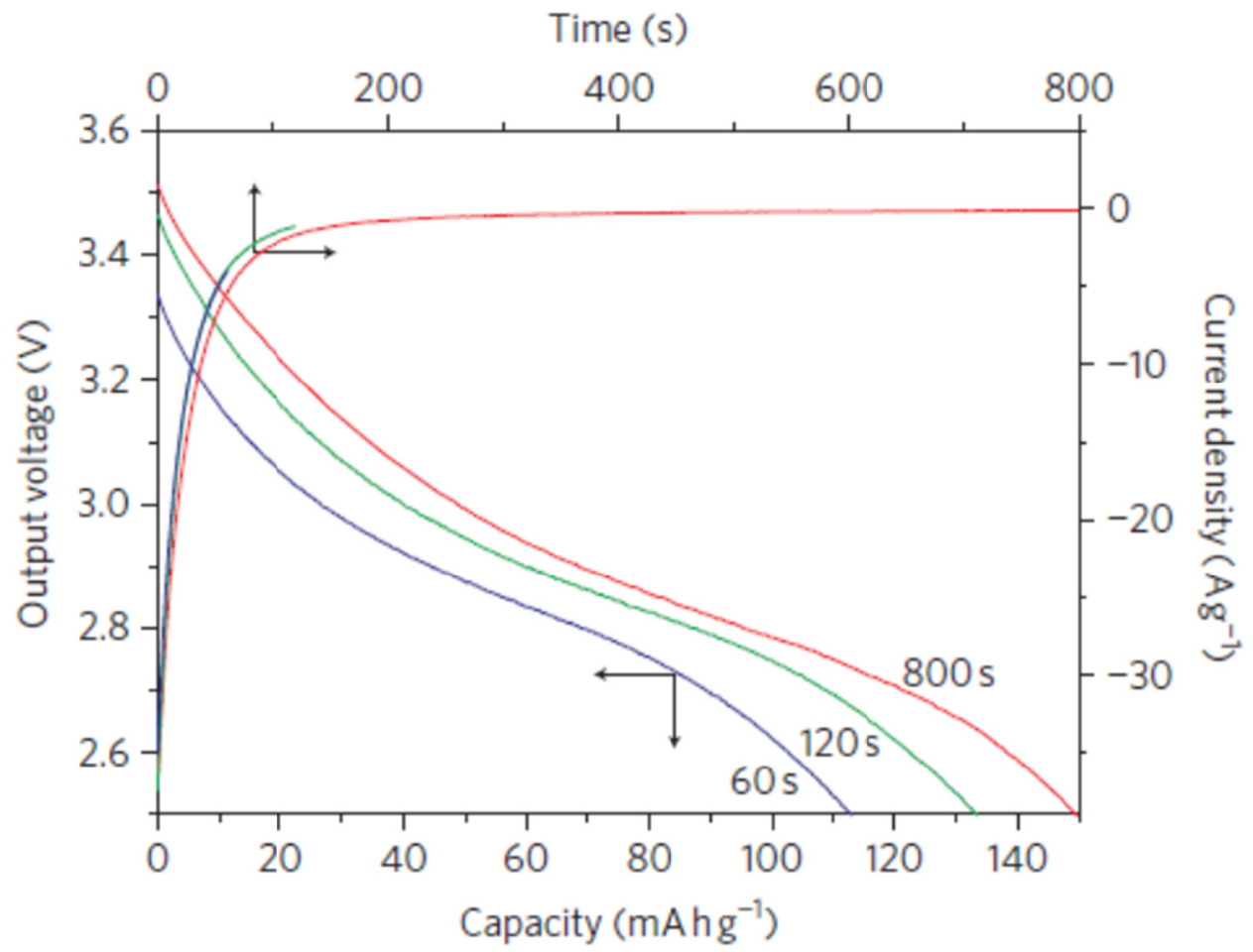

Figure 17. Li-ion battery ultrafast charge behavior. Potentiostatic charging at $3.6 \mathrm{~V}$ for $60 \mathrm{~s}$ (blue), $120 \mathrm{~s}$ (green) and $800 \mathrm{~s}$ (red), and $\sim 3 \mathrm{C}$ galvanostatic discharging of the prototype Li-ion pouch battery after each charging cycle [38].

\subsection{Flexible Li-ion Batteries for Next-Generation Flexible Electronic Devices}

Flexible electronics have attracted great attention to the next-generation portable devices, such as roll/up displays, wearable devices, active radio frequency identification (RFID) tags, integrated circuit smart cards and implantable medical devices as their major applications. Flexible Li-ion batteries (LIB) are used as the energy storage devices for the flexible electronics [44-45]. To date, many leading research groups have combined the nanomaterial-based LIB with the flexible polymer substrates. V. L. Pushparaj et al. fabricated the flexible nanocomposite thin-film battery composed of CNT-cellulose paper electrode (see Figure 18) [46]. 


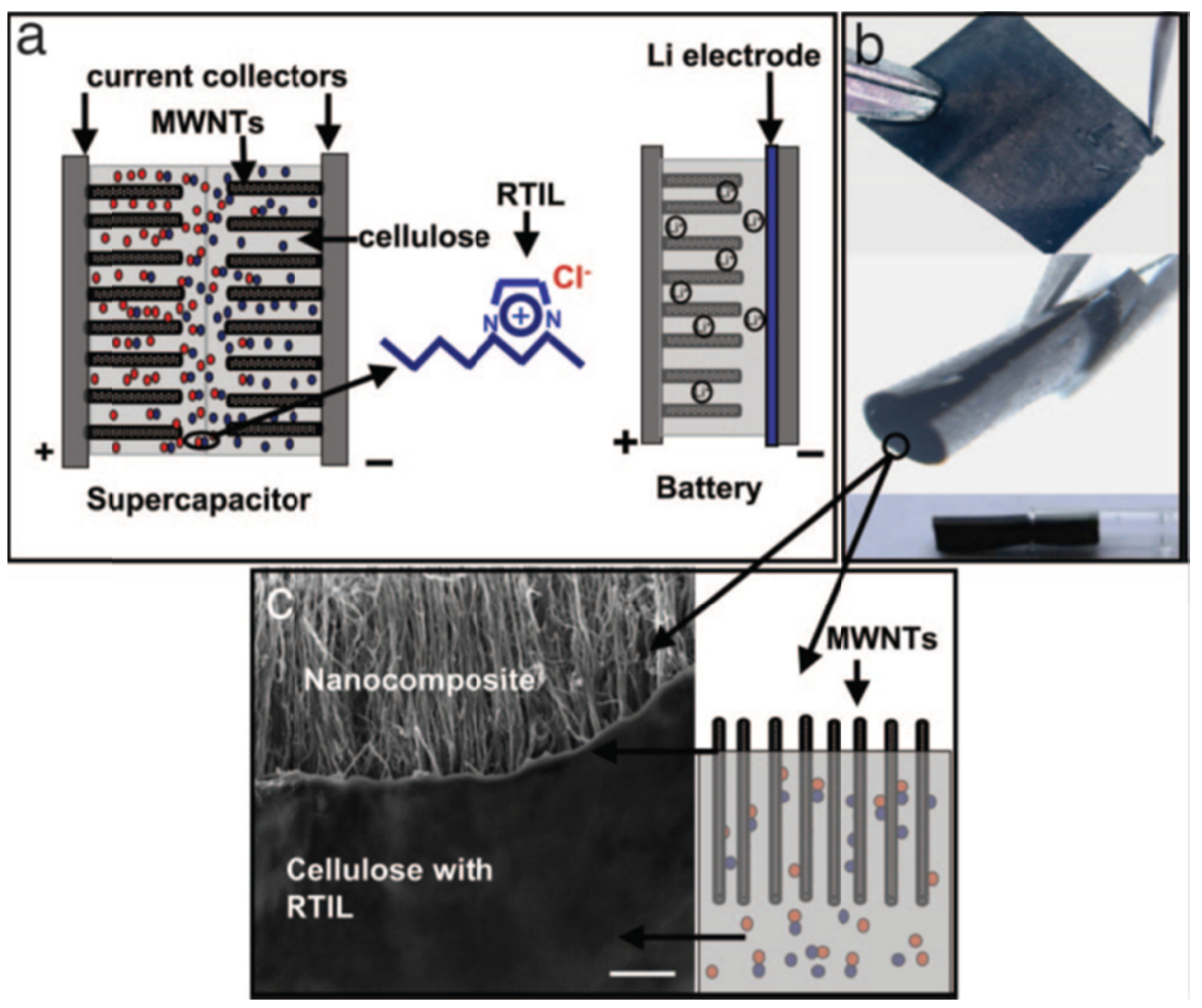

Figure 18. Fabrication of the nanocomposite paper units for supercapacitor and battery. (a) Schematic of the supercapacitor and battery assembled by using nanocomposite film units. The nanocomposite unit comprises RTIL([bmIm][Cl]) and MWCNT embedded inside cellulose paper. A thin extra layer of cellulose covers the top of the MWCNT array. Ti/Au thin film deposited on the exposed MWCNT acts as a current collector. In the battery, a thin Li electrode film is added onto the nanocomposite. (b) Photographs of the nanocomposite units demonstrating mechanical flexibility. Flat sheet (top), partially rolled (middle), and completely rolled up inside a capillary (bottom) are shown. (c) Cross-sectional SEM image of the nanocomposite paper showing MWCNT protruding from the cellulose-RTIL thin films (Scale bar, $2 \mu \mathrm{m}$.). The schematic displays the partial exposure of MWCNT [46]. 
(a)

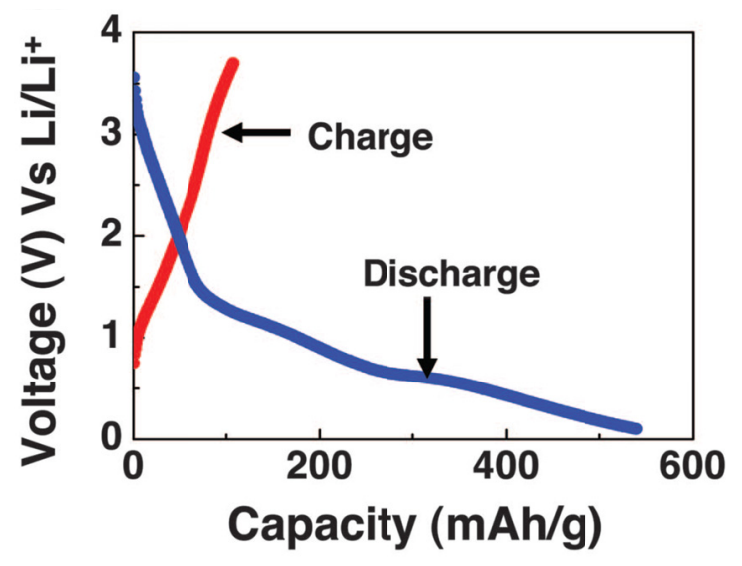

b)

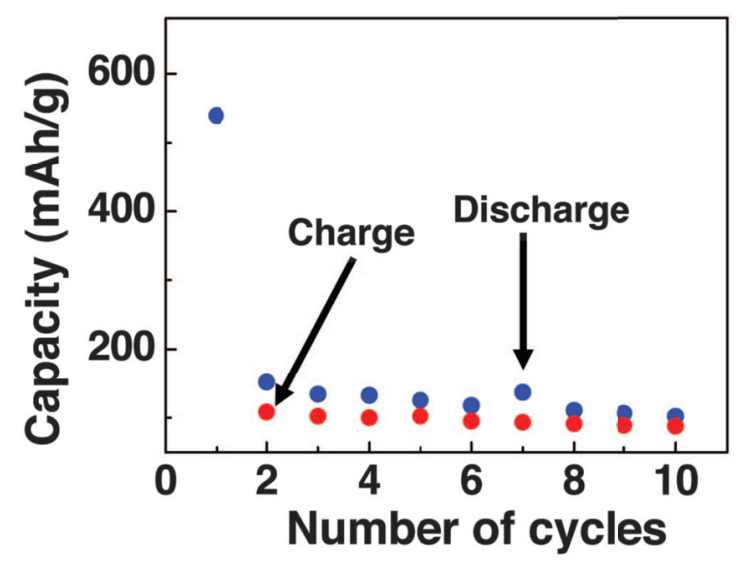

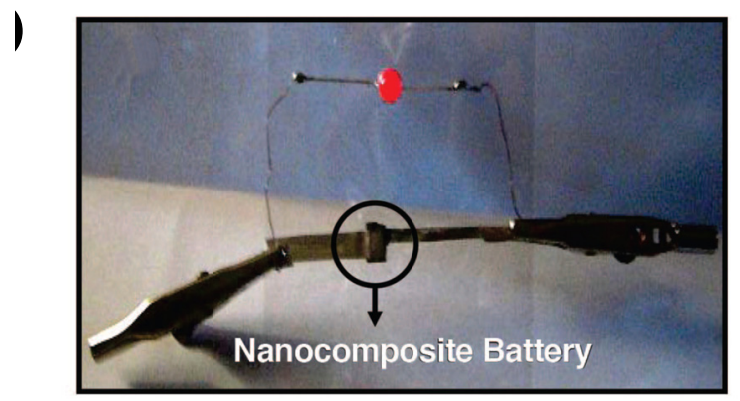

Figure 19. Electrochemical measurements of nanocomposite paper battery. (a) First chargedischarge curves of the nanocomposite thin-film battery cycled between 3.6 and $0.1 \mathrm{~V}$ at a constant current of $10 \mathrm{mAg}^{-1}$. (b) Charge and discharge capacities vs. number of cycles of the nanocomposite thin-film battery. (c) The flexible nanocomposite film battery used to glow a red light-emitting diode (LED). The flexible battery consists of an individual nanocomposite thin film with the Li thin film present on one side as one of the electrodes. The LED glows even when the battery device is rolled up, and the demonstration could be repeated over several tens of cycles at an initial operating voltage of $2.1 \mathrm{~V}[46]$. 
The charge-discharge cycles of the battery were performed between 3.6 and $0.1 \mathrm{~V}$, at a constant current of $10 \mathrm{mAg}^{-1}$. A large irreversible-capacity $\left(430 \mathrm{mAhg}^{-1}\right)$ was observed during the first charge-discharge cycle, as shown in Figure 19(a), and further charge-discharge cycles resulted in a reversible stable capacity of $110 \mathrm{mAhg}^{-1}$ (see Figure 19(b)). It is highlighted that the battery device was operated under full mechanical flexibility. In Figure 19(c), the laminated Liion-based battery device was used to light up a red light-emitting diode, showing its discharge behavior. Also, such demonstration was repeated over several tens of charging and discharging cycles [46].

J. Chen et al. prepared CVD grown CNT-carbon layer (CL) hybrid anodes for a paper LIB device and demonstrated their high reversible, stable specific capacity of $572 \mathrm{mAhg}^{-1}$ after 100 cycles [47]. M. Kaempgen et al. developed single-walled carbon nanotubes (SWCNTs) spray deposited onto PET films and obtained high specific capacitance ranging from $\sim 90$ to $120 \mathrm{~F} / \mathrm{g}$ by using various aqueous electrolytes [48]. 


\section{CHAPTER 3.}

\section{SYNTHESIS OF MWCNT DIRECT GROWN ON METAL SUBSTRATES AND BUTTON CELL ASSEMBLY COMPOSED OF MWCNT-BASED ANODE}

\subsection{Ti/Ni Thin Film Deposition on Cu Substrates by Using an RF Magnetron Sputtering System}

Catalysts play a very significant role in growing MWCNTs and they are generally deposited on substrates by using a sputtering system. Magnetron sputtering is one of the most commercially available techniques for deposition of high-quality films of metal, oxide and non-oxide ceramics and alloys on a substrate under vacuum. The basic fundamental of the sputtering process is the ejection of target material and its subsequent deposition on a substrate. In the case of magnetron sputtering process, the sputtering source or gun is usually a magnetron that uses strong electric and magnetic fields to trap electrons close to the surface of the magnetron, which is known as the target. The electrons conduce through helical paths around the magnetic field lines and undergo more ionizing collisions with gaseous neutrals near the target surface. The whole sputtering process is conducted under a vacuum with an Ar gas environment. Figure 20 illustrates a simple schematic of the magnetron sputtering system. Also, the photograph of the direct current (DC) radio frequency (RF) magnetron sputtering system (AJA International) is shown in Figure 21. The main chamber is under high vacuum condition and the sputtering gun and the substrate holder function as cathode and anode, respectively. The three guns are located at the bottom side of the cylindrical main chamber, whereas the substrate holder and heater are installed under the top side of the chamber. 


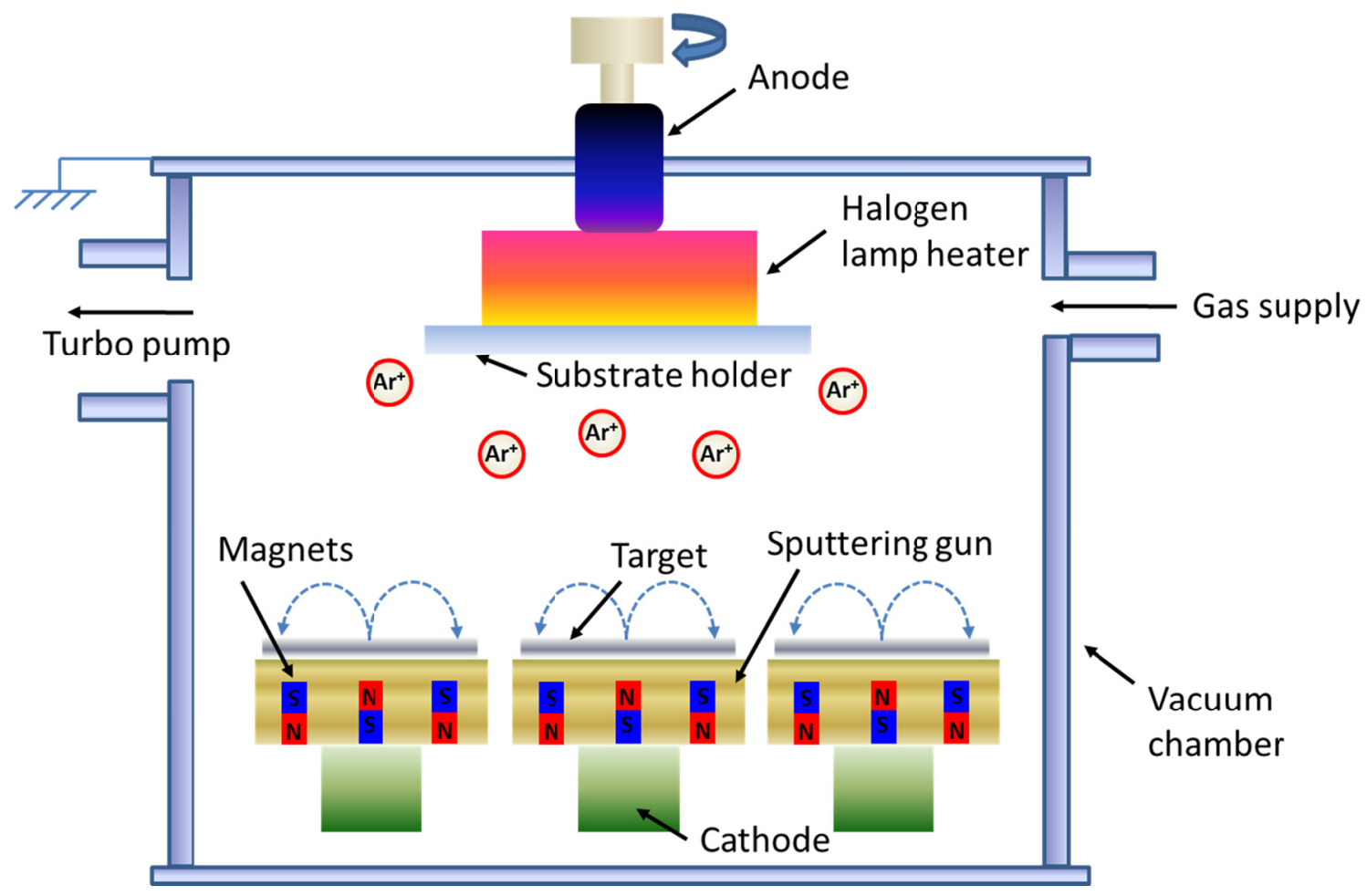

Figure 20. Schematic diagram of a simple magnetron sputtering system.

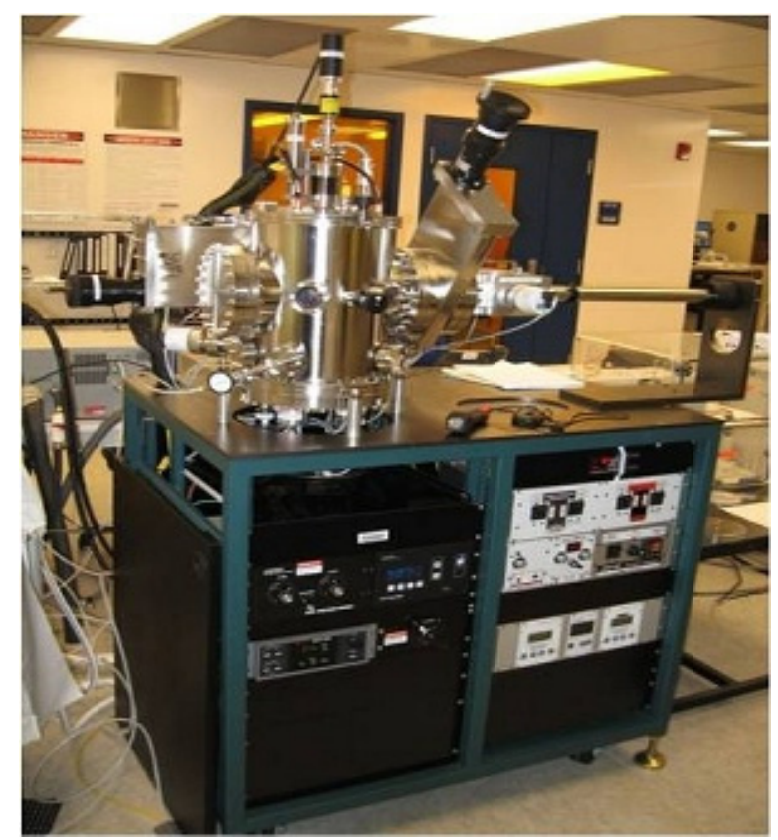

Figure 21. A three-gun magnetron sputtering system (AJA International), used to synthesize catalytic thin films. 
For the sputtering process, the three targets were first installed over the guns, while the substrate holders were facing down towards the target guns [70]. The commonly used catalytic materials of CNT growth were transitional metal, such as Ni, Fe, and Co. In this study, Ni was chosen as catalytic metal deposited on a substrate. Ti was used as a diffusion barrier thin layer to prevent such catalytic metals from being migrated into the substrates and forming compounds. The main chamber was evacuated to the base pressure of $\sim 1 \times 10^{-7}$ Torr by means of connected turbo molecular and roughing pumps. Substrates (e.g. $\mathrm{Cu}$ meshes or foils) were mounted on a disk sample holder, which was subsequently transferred into the main chamber through a load lock system. The sample holder was fixed into the anode and rotated for the homogeneous thin film deposition of the metal catalysts over the substrates. $10 \mathrm{sccm}$ of Ar gas was continuously flowed into the main chamber through the mass flow controller. The plasma was generated at around 25 mTorr pressure in the main chamber with the help of manual gate valve installed between turbo molecular pump and main chamber. This process was performed to increase the Ar gas concentration close to the target metals. A sputtering power of $\sim 75$ Watt was applied to magnetron gun. After generating plasma, the processing pressure was decreased to $\sim 5$ mTorr. Through tuning and loading of matching circuit, reflection power of the sputtering gun was lowered to zero value. Finally, the gun shutter was opened for starting the deposition of the catalytic metal on the substrate. After deposition of catalytic thin film across the desired time line, sputtering power and gas flow were turned off and $\mathrm{Ti}(\sim 10 \mathrm{~nm}$ thickness $) / \mathrm{Ni}(2.5-10 \mathrm{~nm}$ thickness $)$ deposited substrates were taken out through the load lock system [34, 71-72]. Table III represents the standard process condition used for $\mathrm{Ti}$ and $\mathrm{Ni}$ thin film deposition over the $\mathrm{Cu}$ substrate. 
Table III. Standard process conditions used for Ti and Ni thin film deposition.

\begin{tabular}{|c|c|}
\hline Target & Ni and Ti (5N purity) \\
\hline Substrate & Cu mesh and Cu foil \\
\hline Substrate to target distance & $30 \mathrm{~cm}$ \\
\hline Temperature & Room temperature \\
\hline Base pressure & $\sim 10^{-7}$ Torr range \\
\hline Process pressure & 5 mTorr \\
\hline Gas used & Ar \\
\hline Gas flow rate & 75 Watt \\
\hline RF power & 0 Watt \\
\hline Reflection power & \\
\hline
\end{tabular}

We assume that initial nucleation in the Ti/Ni thin film growth follows an island or VolmerWeber growth mode. Island growth proceeds when the growth species (in our case, Ni atoms) are more strongly bonded to each other than to the substrate. Many metal thin films on insulator, alkali halide, graphite, and mica substrates show island nucleation during the initial film deposition. Subsequent growth leads to the calescence of the islands to consequently form a continuous film [73]. 


\subsection{CNT Growth by Using a Catalytic Thermal Chemical Vapor Deposition (CVD)}

\subsubsection{Chemical Vapor Deposition (CVD)}

Chemical vapor deposition (CVD) methods have been successful in making carbon fiber, filament and nanotube materials for the past 20-30 years [74]. Schematic experimental setup for CVD growth is depicted in Figure 22. The growth process involves heating a catalyst material to high temperatures in a tube furnace and flowing a hydrocarbon gas (designated by $\mathrm{C}_{n} \mathrm{H}_{m}$ ) through the tube reactor for a period of time. Materials grown over the catalyst are collected upon cooling the system to room temperature. The key parameters in nanotube CVD growth are the hydrocarbons, catalysts and growth temperature. The active catalytic species are typically transition-metal nanoparticles or thin film, such as $\mathrm{Ni}, \mathrm{Co}$, and $\mathrm{Fe}$ formed on various metal and ceramic substrates. The general nanotube growth mechanism (see Figure 23) in a CVD process involves the dissociation of hydrocarbon molecules catalyzed by the transition metal, and dissolution and saturation of carbon atoms in the metal nanoparticle [75].

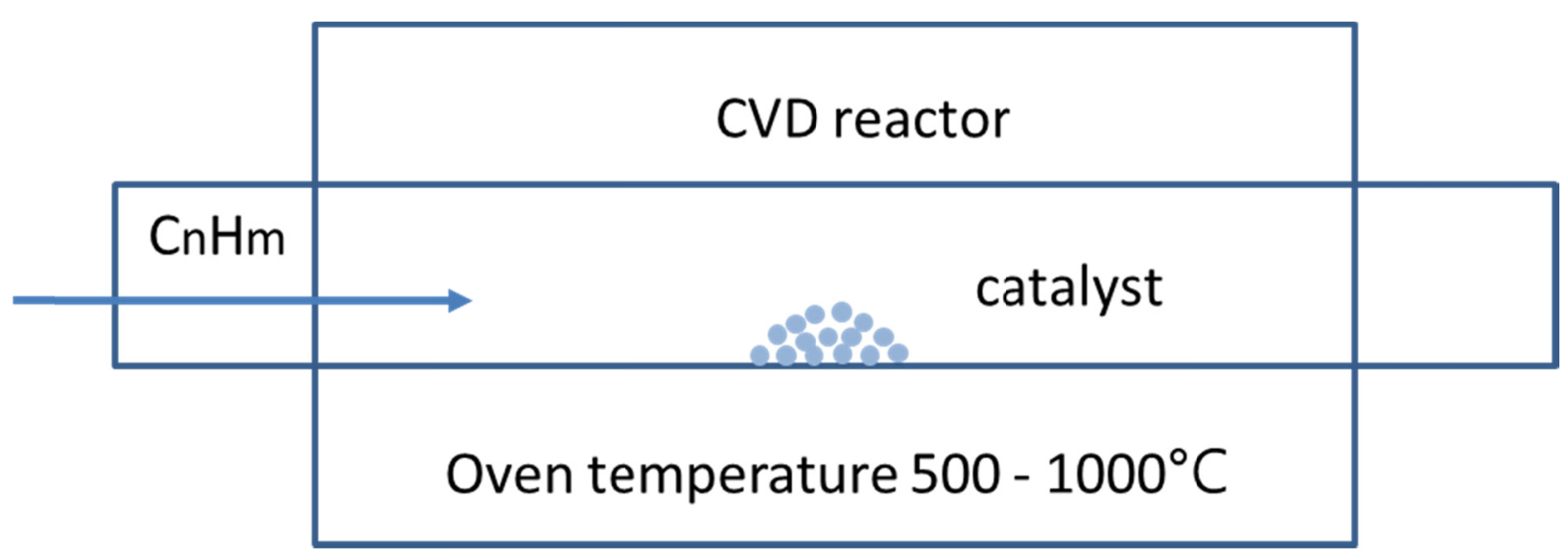

Figure 22. Schematic experimental setup for carbon nanotube growth by means of a catalytic thermal CVD method. 


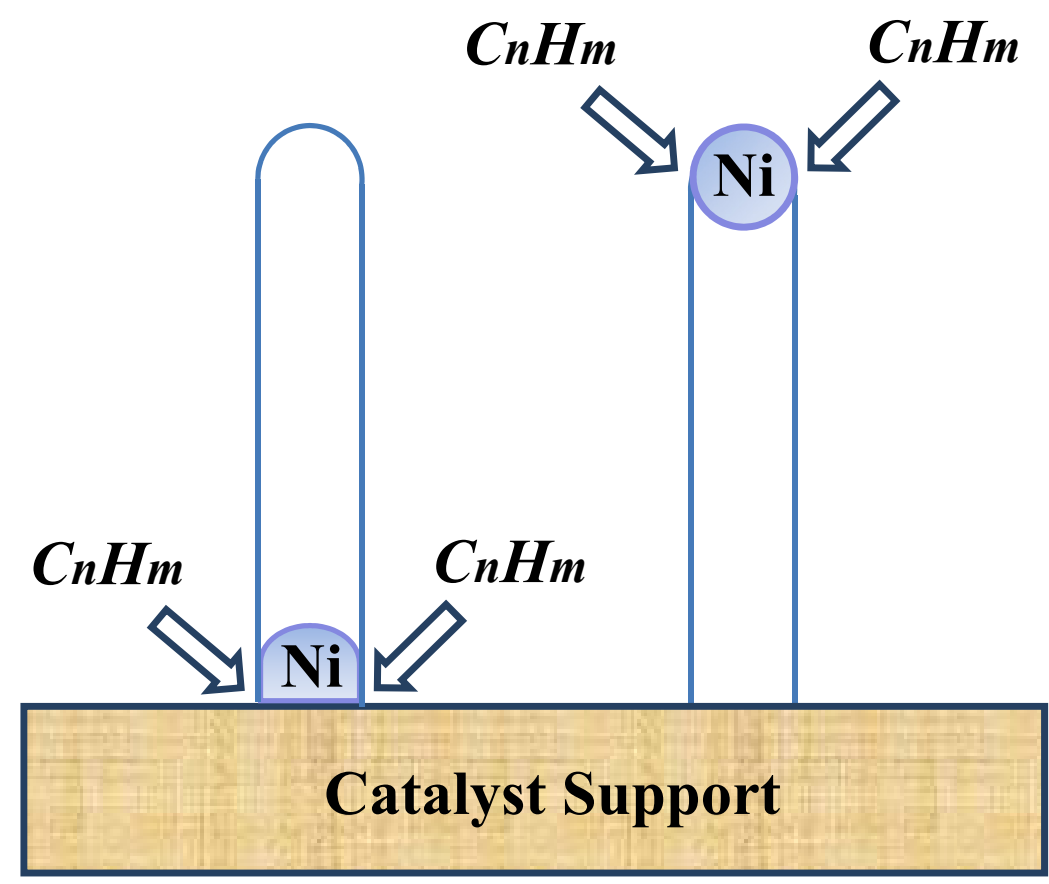

Figure 23. Two general growth modes of carbon nanotube in chemical vapor deposition. Left diagram: base growth mode. Right diagram: tip growth mode [75].

According to the proposed model by R. T. K. Baker et al. we can interpret the nucleation and growth mechanism of the CVD grown 3D MWCNTs on $\mathrm{Cu}$ in the following ways [76]:

(1) At around $\mathrm{CNT}$ growth temperature $700{ }^{\circ} \mathrm{C}$, a Ni thin layer could begin agglomerating as separate, spherical-cap shaped $\mathrm{Ni}$ islands by thermal diffusion of $\mathrm{Ni}$ atoms. Hence, the equivalent diameters of the $\mathrm{Ni}$ islands increased with temperature.

(2) When introduced into the CVD chamber in the $700-750{ }^{\circ} \mathrm{C}$ temperature range, carbon precursor gas (In our case, ethylene $\left.\left(\mathrm{C}_{2} \mathrm{H}_{4}\right)\right)$ was decomposed and the released carbon atoms 
were diffused into the Ni islands from their surface to core due to the thermal gradient in the $\mathrm{Ni}$ islands.

(3) The dissolution of carbon into the Ni islands ceased after passing their solubility limit of carbon and began precipitating at the trailing ends of the Ni islands and growing MWCNTs.

Since the decomposition of the ethylene precursor gas exothermically occurred, a temperature gradient existed across the Ni catalyst islands. The solubility was temperature dependent and showed the highest degree at the front-exposed surfaces of the $\mathrm{Ni}$ and the lowest one at the colder zone on the rear surfaces of the $\mathrm{Ni}$ where were close to the $\mathrm{Cu}$ mesh substrate. In addition, according to the bonding strength between the $\mathrm{Ni}$ catalyst and $\mathrm{TiC}$ underlayer/Cu substrate, the tip or base growth mode can be determined, as shown in Figure 23. On the left, stronger chemisorption between catalyst and substrate facilitates base growth of MWCNTs. Tip growth mode, drawn on the right, is predominant when the chemisorption does not appear to be strong enough to anchor catalyst to substrate [75]. However, a major disadvantage for CVD grown MWCNTs has been the high defect densities in their structures. The defective nature of CVD grown MWCNTs remains to be thoroughly understood, but is most likely due to relatively low growth temperature, which does not provide sufficient thermal energy to anneal nanotubes into perfectly crystalline structures.

For CNT growth by a catalytic thermal CVD process, catalyst deposited substrates are to be inserted into a quartz tube reactor in a CVD system. The internal structure of our CVD chamber is similar to the schematic diagram, as presented in Figure 22. Figure 24 shows our CVD systems (Figure 24(a) FirstNano; Figure 24(b) A-Tech system, Korea) used for MWCNT growth. The typical CNT growth conditions are listed in Table IV. 
Table IV. The summary of key conditions for CNT growth through a catalytic thermal CVD method

\begin{tabular}{|c|c|c|c|}
\hline Step & Temperature & Gas & Flow rate (SCCM) \\
\hline Ramping & Room temperature to $300^{\circ} \mathrm{C}$ & Argon & 600 \\
\hline H2 etching & $300^{\circ} \mathrm{C}$ & Hydrogen & 300 \\
\hline Ramping & $300^{\circ} \mathrm{C}$ to $750^{\circ} \mathrm{C}$ & Argon & 600 \\
\hline Growth & $750^{\circ} \mathrm{C}$ & Hydrogen : Ethylene $(\mathrm{C} 2 \mathrm{H} 4)$ & $300: 600$ \\
\hline Cooling & $750^{\circ} \mathrm{C}$ to Room temperature & Argon & 600 \\
\hline
\end{tabular}

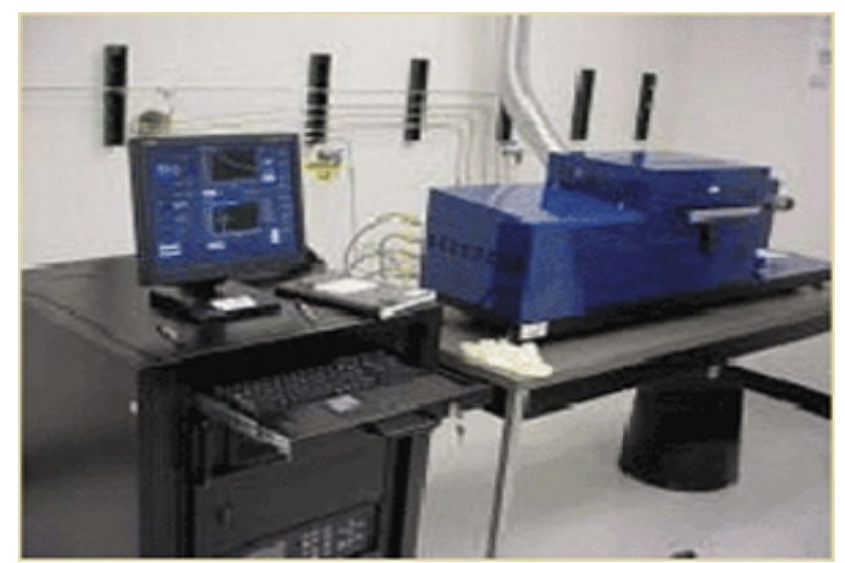

(a)

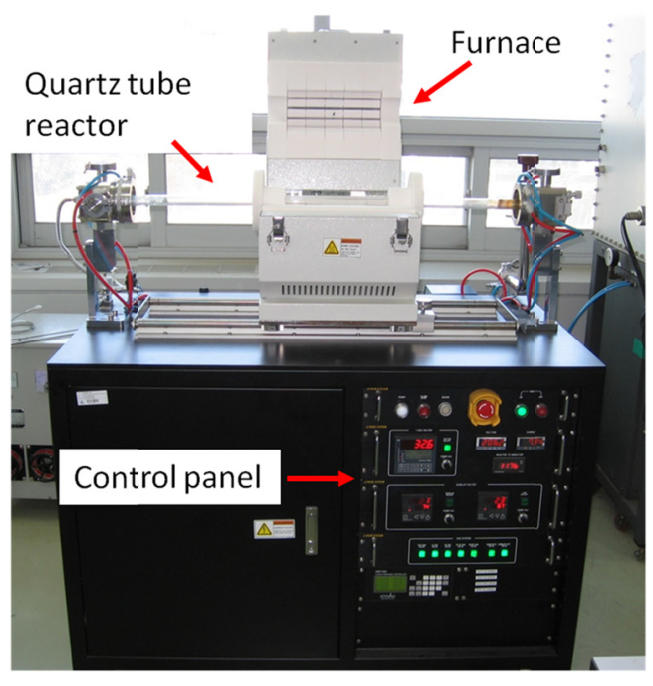

(b)

Figure 24. Thermal chemical vapor deposition systems manufactured by (a) FirstNano Inc., and (b) A-Tech system Inc.

To explore the morphology variation of the 3D MWCNTs directly grown on $\mathrm{Cu}$, we controlled the growth conditions, such as growth temperature and time, and deposition time for catalytic Ni thin layer. As seen in Table V, the most probable size (which is noted in each image) 
of the Ni catalyst islands (clusters) increased with not only the temperature due to higher mobility of the atoms but also the thickness of $\mathrm{Ni}$ thin layer due to larger volume for the formation of $\mathrm{Ni}$ islands. The sizes of the Ni clusters were measured by ImageJ program, and a size distribution of the clusters existed (see Figure 25). Upon heating, the sputter-deposited 2-dimensional Ni thin film underwent the compressed stress due to the mismatch of the linear thermal expansion coefficients $(\alpha)$ between $\mathrm{Ni}\left(13 \times 10^{-6} /{ }^{\circ} \mathrm{C}\right)$ and $\mathrm{Cu}\left(17 \times 10^{-6} /{ }^{\circ} \mathrm{C}\right)$. The compressed strained $\mathrm{Ni}$ thin layers tended to transform into separate semi-spherical islands to release internal strain energy. On the other hand, the Ti layer did not form clusters due to its lower $\left(8.5 \times 10^{-6} /{ }^{\circ} \mathrm{C}\right)$ linear thermal expansion coefficient (lower strain energy) and higher melting point (lower mobility of the atoms at a given temperature) [77]. It is well understood that the segregation and precipitation of solutes favorably occur at defect sites such as dislocations and grain boundaries, where the stress fields are more intense. Therefore, polycrystalline Ni islands provide many sites for carbon nanotube growth. For lower temperature (e.g., $700{ }^{\circ} \mathrm{C}$ ), the solubility of carbon into the Ni islands and diffusion coefficient of carbon atoms are lower; thus, carbon nanotube nucleation starts from smaller grains of the Ni islands. However, for higher temperature (e.g., $750{ }^{\circ} \mathrm{C}$ ), the average size of the catalytic Ni islands increases due to larger coalescence induced by faster thermal diffusion of $\mathrm{Ni}$ atoms. The larger surface of the $\mathrm{Ni}$ islands allows a larger intake of carbon atoms, and diffusive flux of carbon through Ni islands becomes accelerated. Consequently, carbon nanotubes with larger diameters can be formed at higher growth temperature [78-79].

According to basic theory, when the average size of catalytic clusters is much larger than the diffusion length of carbon atoms, carbon atoms cannot migrate to the rear side of the clusters for CNT growth. Conversely, in the case of a thin region, most of the clusters are smaller than the diffusion length so that they can effectively assist CNT growth. Therefore, in our case, the thicknesses of $\mathrm{Ni}$ thin layer in the region of 2.5-10 $\mathrm{nm}$ belong to the transitional regions between those two extremes. Also, the diameters of as-grown MWCNTs from the Ni thin layer with 
thickness $(>4 \mathrm{~nm})$ showed no significant difference. However, at the elevated temperature from $700{ }^{\circ} \mathrm{C}$ to $750{ }^{\circ} \mathrm{C}$, the diameters of as-grown MWCNTs considerably increased at each thickness of the Ni thin layer $(2.5,5,7.5$, and $10 \mathrm{~nm})$. Therefore, we speculate that the temperature effect

Table V. The variation of diameter of catalyst Ni islands with the CNT growth conditions. Ethylene gas as carbon precursor was flowed into the chamber for one minute. Numerical values noted in the top-left of each image represent the most probable size of the Ni islands.

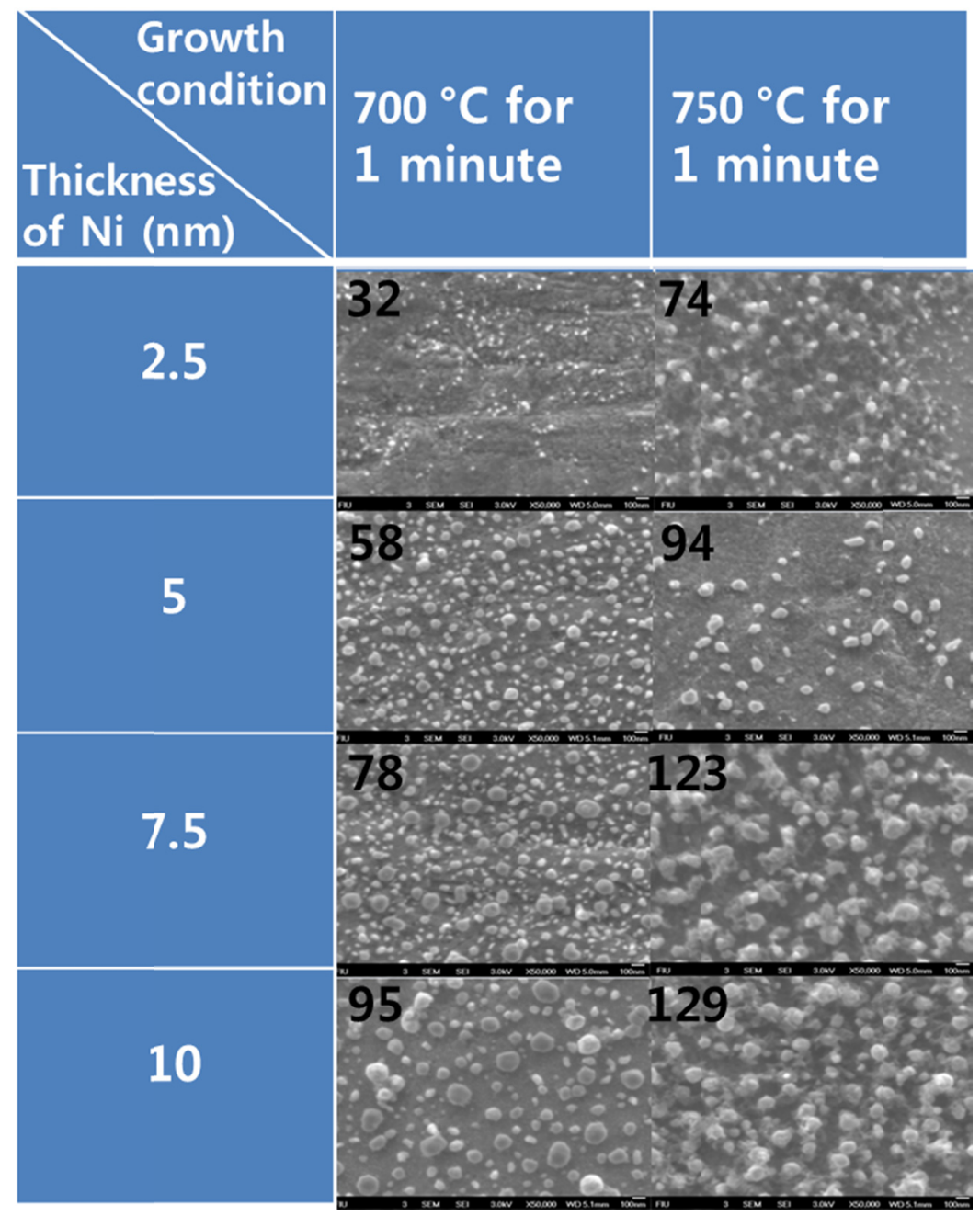




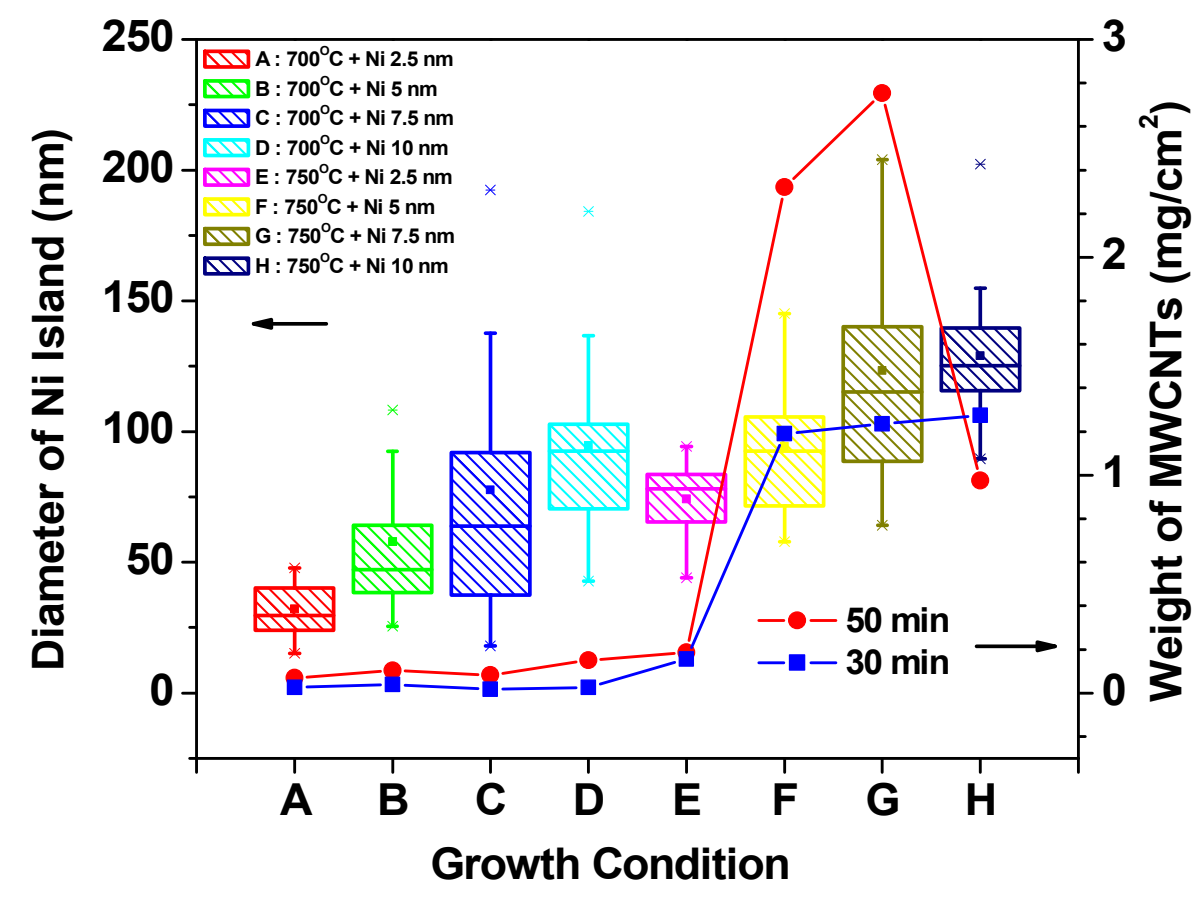

Figure 25. The variation of the diameters of catalyst $\mathrm{Ni}$ islands and the areal weights of MWCNTs with CNT growth conditions

on the diameters of MWCNTs was more predominant, compared to the effect of the thickness of Ni thin layer. C. Ducati et al. proposed an equation to express the growth rate (R) of MWCNTs at temperature $(\mathrm{T})$ with various parameters, as follows [78]:

$$
R \propto \frac{D_{0} S_{0} \exp \left(-\frac{Q+q}{k T}\right)}{x}
$$

where $x$ is the diffusion distance or the grain size, $D_{0}$ and $S_{0}$ are the diffusivity and solubility prefactors, and $Q$ and $q$ are the activation energies for diffusivity and solubility, respectively. 
We confirmed that the morphologies of MWCNTs shown in the SEM images of Table VI could be varied from each growth condition. As the temperature increases, the decomposition and diffusion rates of carbons increased and the growth rate of MWCNTs proportionally increased. As a result, the areal density of MWCNTs grown on by $750{ }^{\circ} \mathrm{C}$ for 50 minutes and $7.5 \mathrm{~nm}$ thickness of Ni thin layer was $1,800 \%$ higher than that of MWCNTs grown by $700{ }^{\circ} \mathrm{C}$ for 50 minutes and 2.5 to $10 \mathrm{~nm}$ thickness of Ni thin layer (see Figure 25). Also, the diffusion rate of $\mathrm{Ni}$ atoms on the Ti barrier layer deposited on $\mathrm{Cu}$ substrate promoted facilitating the agglomeration of Ni nanoparticles. The size of the agglomerated Ni islands usually determined the diameter of MWCNTs in CVD growth. In the case of $700{ }^{\circ} \mathrm{C}$, the diameters of MWCNTs were less than 100 $\mathrm{nm}$, whereas those of MWCNTs grown at $750{ }^{\circ} \mathrm{C}$ were in the range of $200-300 \mathrm{~nm}$. The remarkable variation in the diameters could be found between the Ni thin layers with 2.5 and 5 $\mathrm{nm}$ thicknesses. In the case of $2.5 \mathrm{~nm}$ thickness, the average diameter of as-grown MWCNTs was smaller than $50 \mathrm{~nm}$ even though they showed a broad diameter distribution, while, in the case of the thicknesses higher than $5 \mathrm{~nm}$, the average diameters of as-grown MWCNTs were in the range of 200 and $300 \mathrm{~nm}$. According to those results, we assumed that the temperature range from 700 to $750{ }^{\circ} \mathrm{C}$ was critical enough to affect the diameters of the as-grown MWCNTs. 
Table VI. The variation of morphology of 3D MWCNTs on $\mathrm{Cu}$ obtained from the different growth conditions.

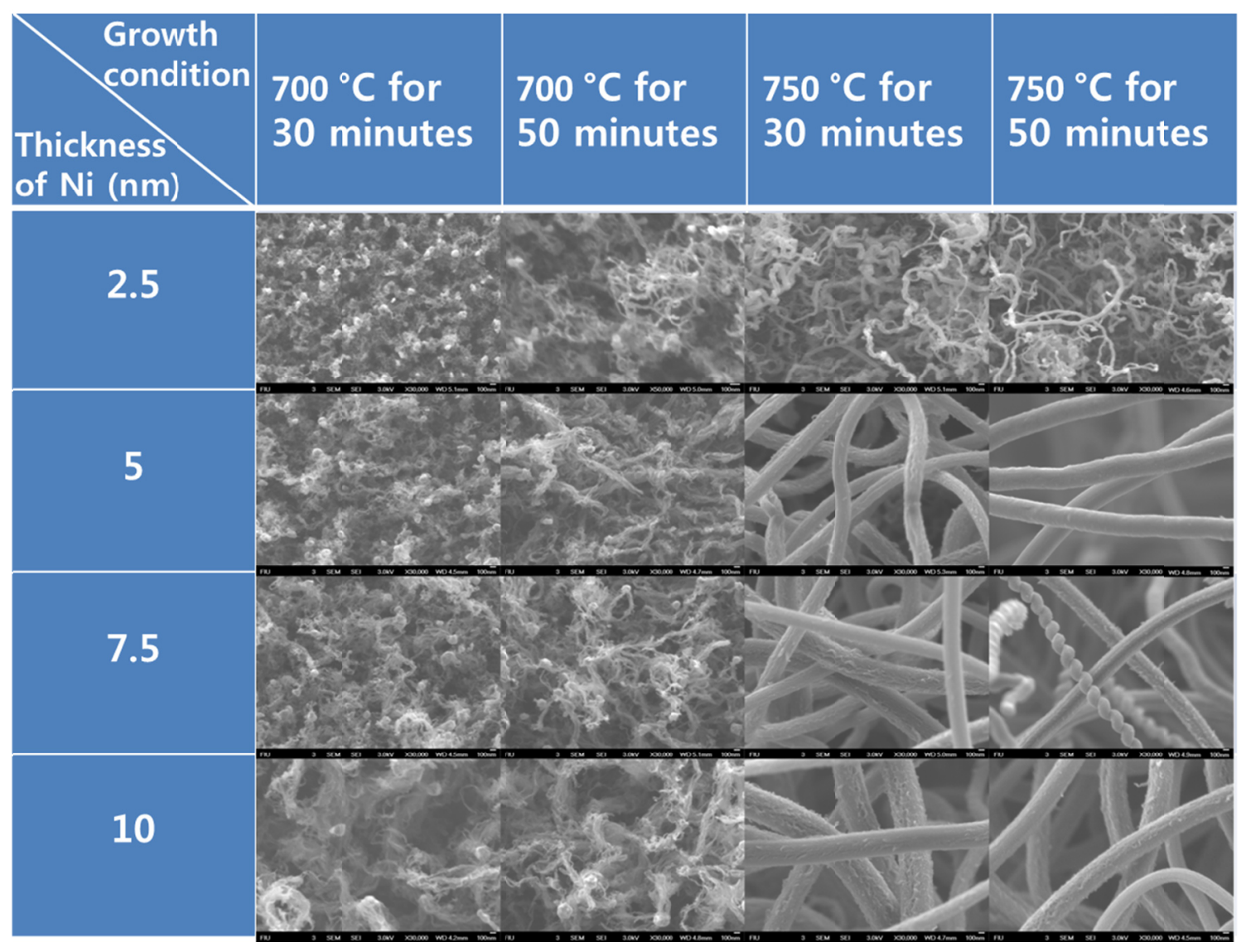

Moreover, we chose the three different MWCNT images and referred to each one as S-CNT (T) (The smaller diameter CNTs grown at Ni $10 \mathrm{~nm}$ thickness and $725{ }^{\circ} \mathrm{C}$ temperature), S-CNT (C) (The smaller diameter CNTs grown at Ni $2.5 \mathrm{~nm}$ thickness and $750{ }^{\circ} \mathrm{C}$ temperature), and LCNT (The larger diameter CNTs grown at Ni $10 \mathrm{~nm}$ thickness and $750{ }^{\circ} \mathrm{C}$ temperature), respectively. In addition, from the HRTEM images illustrated in Figure 26, we could find that the average diameters of S-CNT (C), S-CNT (T), and L-CNT were $21 \mathrm{~nm}, 32 \mathrm{~nm}$, and $279 \mathrm{~nm}$, respectively. We would like to mention that the blur image indicated by the red arrow represents 
the depth structure of MWCNT network with the higher density (see the upper image of Figure 26(b)). Since their internal structures were composed of the lower-ordered graphene layer stacking, they were considered as turbostratic structure. However, the internal structure of S-CNT (C) was similar to herringbone structure. Recently, Y.Y. Wang et al. demonstrated the effect of the thickness of Fe catalytic thin layer (from 0.3 to $20 \mathrm{~nm}$ ) deposited on $\mathrm{SiO}_{2} / \mathrm{Si}(100)$ substrate for the morphological variation in the internal structure of MWCNTs (see Figure 27) [80]. Compared to their results, our cases did not show the distinguishable structural variation as a function of the thickness of catalytic Ni thin layer.

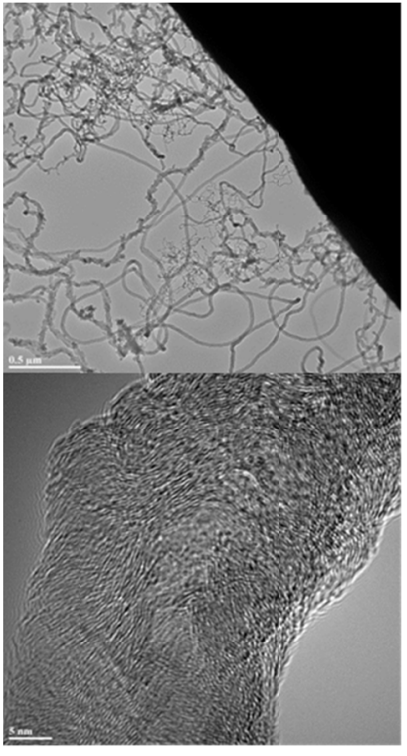

(a)

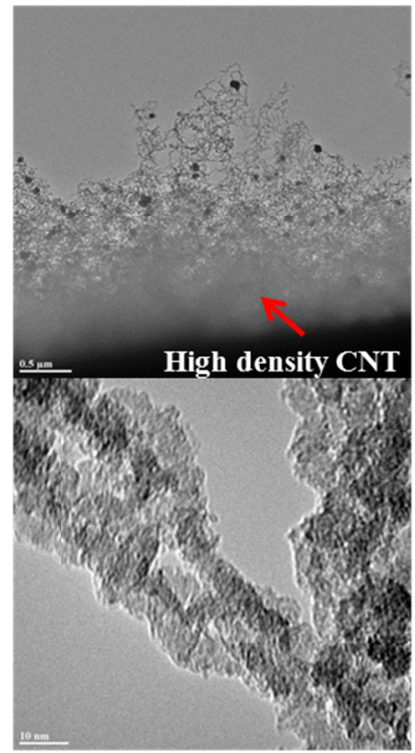

(b)

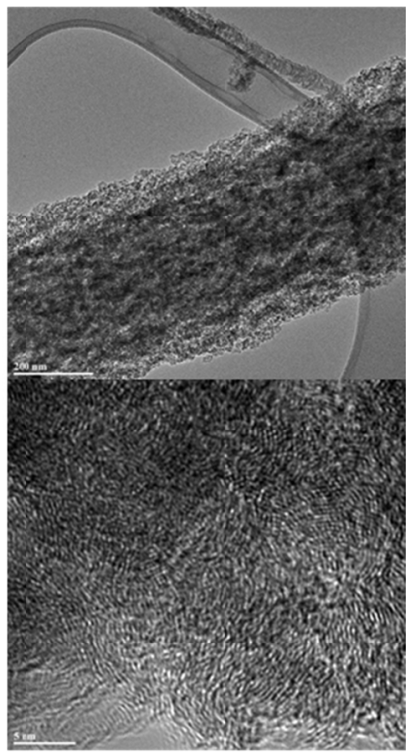

(c)

Figure 26. HRTEM images of the morphology of 3D MWCNTs synthesized at the different growth conditions designated by (a) S-CNT (C), (b) S-CNT (H), and (c) L-CNT 

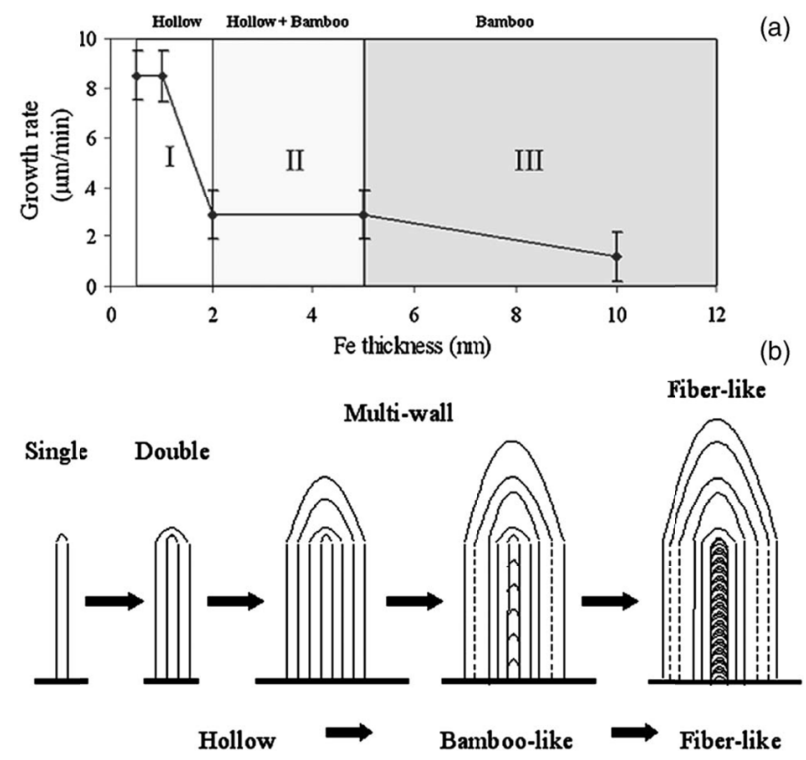

Figure 27. (a) Plot of the CNT film growth rate vs. Fe thickness where three regions (I, II, and III) with different internal structures are observed. (b) A schematic of the internal structure transition from hollow to bamboolike [80]

\subsection{Button Cell Assembly Under Ar Gas Flow Glove Box}

The coin type of cell assembly is crucial for the performance of Li-ion batteries. As seen in Figure 28(b), the components of coin cell assembly are electrode materials, Li metal, stainless steel (SS) caps, separator, spring and spacer. The very low oxygen and humidity are less than 1 ppm level, which are automatically controlled in the glove box by the combined functions of evacuation and Ar gas flowing. Figure 28 shows photograph images of the glove box installed in Nanomaterials and Nanodevice Lab at Hanyang University in Korea and button cell assembly components. Electrode materials are placed on the stainless steel bottom cap and separator is place on the electrode. Li metal is put on the separator, followed by putting the spring and spacer on the Li metal. Finally, SS top cap is covered and coin cell assembly is completed by pressing a crimper (CR 2032, Honsen Corp.). 


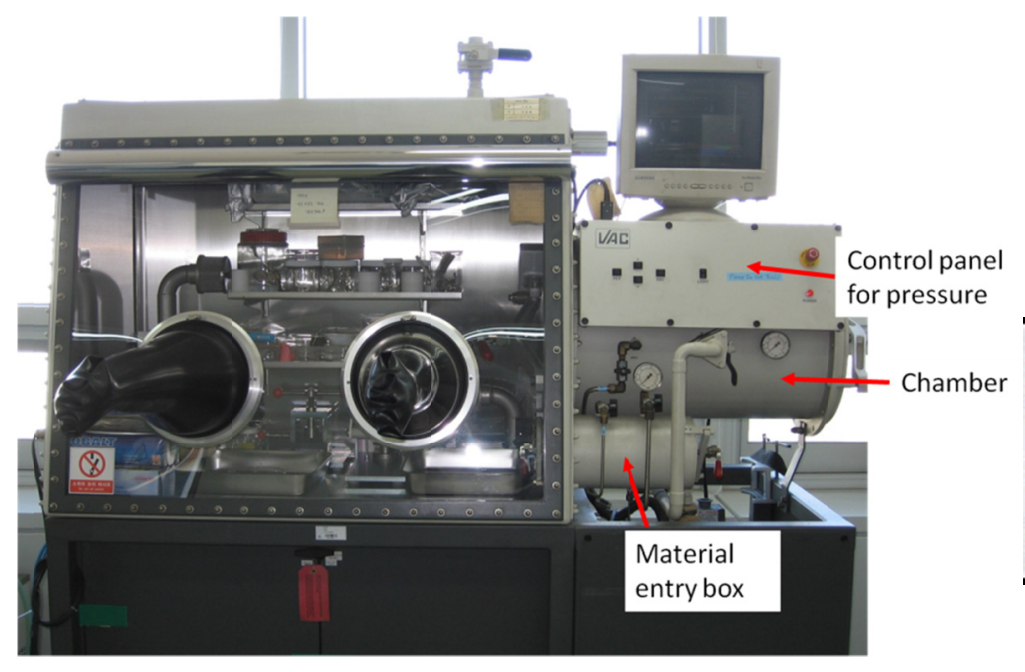

(a)

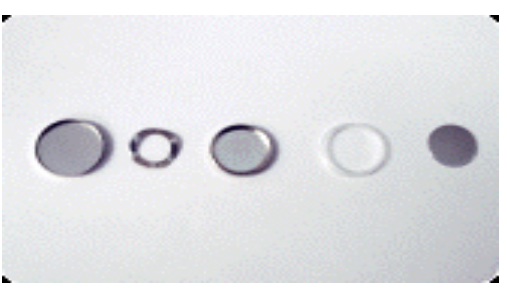

(b)

Figure 28. Photograph images of (a) a glove box for LIB button cell assembly and (b) components of the button cell

\subsection{Battery Performance Test}

The charge-discharge tests of the cells were performed in TOSCAT 3100U and MACCOR multi-channel battery testing units, at a constant temperature of $30^{\circ} \mathrm{C}$ and room temperature, in galvanostatic (constant current density) mode. The cells were cycled in the voltage range 3.0 $0.01 \mathrm{~V}$, recording a current value in each $10 \mathrm{mV}$ step, at a slow rate $(0.1 \mathrm{C})$ during the initial formation process and at various C-rates in the following cycles.

\subsection{Analytical Techniques}

Morphology and structural properties of the prepared anode structures were carefully investigated using field emission scanning electron microscopes (FESEM) (JEOL, JSM-7000F), 
an energy dispersive spectroscope (EDS) (Thermo Electron Corporation, NORAN System SIX), a Raman spectrometer (laser source : $\operatorname{Ar}^{+}$laser with $\lambda=514 \mathrm{~nm}, 33 \mathrm{~mW}$ power) and micro Raman system (UniG2D, Korea; see Figure 29) (laser source : low noise $532 \mathrm{~nm}$ Diode-pumped solid-state (DPSS) laser $(3.7 \mathrm{~mW})$; software : iDus CCD detector) and a field emission transmission electron microscope (FETEM) (FEI, TECHNAI F20).

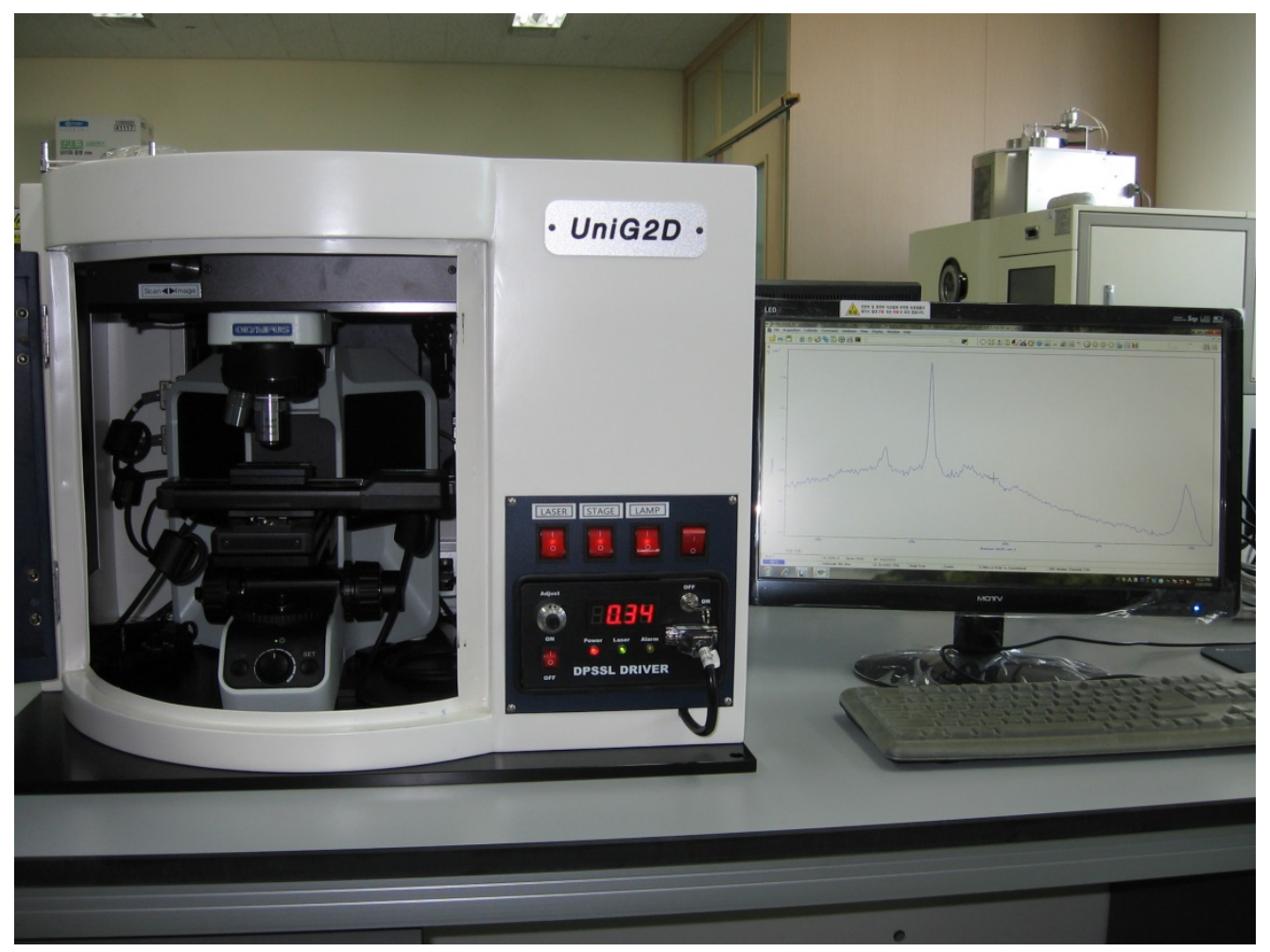

Figure 29. A photograph of micro Raman system 


\section{CHAPTER 4.}

\section{3-DIMENSIONAL CARBON NANOTUBE FOR LI-ION BATTERY ANODE}

\subsection{Introduction}

Carbon nanotubes (CNTs) have been widely researched into the application of LIB anode materials due to high surface area, short diffusion length of $\mathrm{Li}^{+}$ions, and high electrical conductivity [54]. From our team, previous researches showed excellent performance of MWCNT-based binder-free anodes in terms of the high specific capacity, excellent rate capability, and almost no capacity degradation during a prolong cycling [34-35]. However, nanostructured carbon materials are known as low-density materials, which tend to show low volumetric capacity and low volumetric energy and power density. Hence, increase in loading density of MWCNTs as active materials should be most significantly dealt with in the practical application. Very recently, Y. Gogotsi et al. argued that carbon-based nanomaterials have a critical limitation with respect to the low area mass density [36]. As a result, even the high gravimetric energy density may bring about scale-up issues and may not be useful for the potential application to the development of electric vehicles (EVs).

To address this issue, we propose a novel architecture of 3D $\mathrm{Cu}$ current collector, which can function as increasing surface area to accommodate larger area or volumetric mass density of MWCNTs over the uniformly arrayed patterns in the 3D structure; thus, resulting in higher specific capacity and C-rate capability. To our best knowledge, much work has been done to enhance electrochemical performance of Li-ion batteries and supercapacitors by using 3D structured current collectors, such as carbon papers [37], a self-assembled 3D bicontinuous nanoarchitecture [38], aluminum nanorods [39], and nanoporous nickel [40]. Z. Yan et al. demonstrated that a self-assembled 3D bicontinuous nanoarchitecture could realize not only the 
high volume fraction of nanostructured electrolytically active materials ( $\mathrm{NiOOH} / \mathrm{Nickel}$ and $\mathrm{MnO}_{2}$ cathodes) but also the efficient ion and electron transport [38]. Additionally, atomic layer deposition (ALD) coated $\mathrm{TiO}_{2}$ anodes on 3D aluminum nanorod current collectors showed a tenfold increase in the theoretical area capacity $\left(0.0112 \mathrm{mAhcm}^{-2}\right)$, compared to that from the same anodes on 2D flat aluminum plate and high-rate capability (the capacity ratios at $10 \mathrm{C} / 0.5 \mathrm{C}$ and $20 \mathrm{C} / 0.5 \mathrm{C}$ of the $3 \mathrm{D}$ anode were 0.4 and 0.35 , respectively.) [39].

Currently, the various types of hybrid anode structures have been proposed and synthesized to expect the synergetic combination of two different types of nanomaterials for higher electrochemical performance. Among the strong candidates of anode materials in the preferable combinations, an MWCNTs/Si hybrid structure can be significantly considered due to excellent mechanical resilience of MWCNTs against large volume expansion/constriction of Si during the lithiation/delithiation process and good bonding strength between MWCNTs and Si. There have been several reports on MWCNTs/Si composite structures; however, data employing either the $\mathrm{SiH}_{4}$ CVD method [41] or sputter deposition [42] were particularly used for this dissertation.

In this chapter, we present a novel concept 3D anode system, comprised of MWCNTs directly grown on 3D $\mathrm{Cu}$ mesh using a catalytic thermal CVD method [81]. Electrochemical performances of the 3D anode structure are compared with those of MWCNTs directly grown on 2D Cu foil. Furthermore, enhanced electrochemical properties of a-Si/MWCNTs hybrid structure, synthesized on 3D Cu mesh using CVD and sputtering deposition methods, are presented. Finally, we discuss the morphology and structure of as-grown MWCNTs and a-Si/MWCNTs hybrid anode structures and their role in the electrochemical performance. 


\subsection{Results and Discussion}

\subsubsection{Experimental Details}

A Cu mesh (TWP Inc.) with average dimensions of $50 \mu \mathrm{m}$ thickness and $65 \mu \mathrm{m}$ hole size was prepared. In parallel, a $50 \mu \mathrm{m}$ thick pure $\mathrm{Cu}$ foil (Nimrod Hall Copper, $99.9 \%$ purity) was also employed. Both types of samples were used as substrates for depositing $\mathrm{Ti}$ (underlayer)/Ni (catalyst) thin film through an RF and DC magnetron sputtering system. These Ti/Ni thin film deposited samples were cut to $14 \mathrm{~mm}$ diameter disc shape for 2032 button cell assembly, before inserting into a thermal CVD system for direct MWCNT growth. During CVD, samples were heated very rapidly, under an inert Ar gas environment, to the growth temperature of $750{ }^{\circ} \mathrm{C}$, and MWCNT growth began with flow of a mixture of ethylene $\left(\mathrm{C}_{2} \mathrm{H}_{4}\right)$ and hydrogen $\left(\mathrm{H}_{2}\right)$ gas $(1: 2$ volume ratio) in the chamber. After 50 minutes of growth, the samples were cooled to room temperature within the furnace under an Ar gas envelope. Amorphous Si (a-Si) was deposited further on the as-grown MWCNT samples using the sputtering system with the incorporation of Ti adhesion layer in order to enhance bonding strength between a-Si and MWCNTs. Weights of samples were measured before and after CVD growth to exactly identify weights of the active materials (i.e. MWCNTs and a-Si thin layer).

Morphology and structural properties of the prepared anode structures were carefully investigated using field emission scanning electron microscopes (FESEM) (JEOL, JSM-7000F), an energy dispersive spectroscope (EDS) (Thermo Electron Corporation, NORAN System SIX), a Raman spectrometer $\left(\mathrm{Ar}^{+}\right.$laser with $\lambda=514 \mathrm{~nm}, 33 \mathrm{~mW}$ power) and a field emission transmission electron microscope (FETEM) (FEI, TECHNAI F20). Electrochemical performance for these anodic materials was conducted in a typical coin cell (half cell). The cells were assembled in a CR2032 press. The complete cell assembly was carried out in an argon glovebox under extremely low levels of oxygen and humidity (both individually $<0.1 \mathrm{ppm}$ ). A pure Li 
(purity, $99.9 \%)$ metal foil (150 $\mu \mathrm{m}$ thickness) was used as both the reference electrode and counter electrode, while the MWCNTs on 3D Cu mesh, the MWCNTs on 2D Cu foil and the aSi/MWCNTs composite on 3D Cu mesh were used as the working electrodes. All the coin cells employed a solution 1.0 $\mathrm{M} \mathrm{LiPF}_{6}$ in EC-DEC (ethylene carbonate : diethyl carbonate, 1:1 in volume ratio) as the electrolyte and a typical polypropylene-polyethylene material (Celgard 3401) as the separator. The charge-discharge tests of the cells were performed in TOSCAT $3100 \mathrm{U}$ multi-channel battery testing unit, at a constant temperature of $30^{\circ} \mathrm{C}$, in galvanostatic (constant current density) mode. The cells were cycled in the voltage range $3.0-0.01 \mathrm{~V}$, recording a current value in each $10 \mathrm{mV}$ step, at a slow rate $(0.1 \mathrm{C})$ during the initial formation process and at various $\mathrm{C}$-rates in the following cycles.

\subsubsection{Theoretical Calculation of the Surface Area of 3D Cu Mesh}

The purpose to introduce 3D $\mathrm{Cu}$ mesh as current collector was to increase in surface area for the growth of MWCNTs, thus leading to greater amount of $\mathrm{Li}^{+}$intake into them, as compared to 2D $\mathrm{Cu}$ foil. The calculation procedure of the theoretical surface area of 3D $\mathrm{Cu}$ mesh consists of following steps.

\section{Step 1.}

Making a real $\mathrm{Cu}$ mesh simplifies the geometry arrayed by numerous rectangular cuboid holes separated by regular and repetitive rectangular barriers, as shown in Figure 30. 


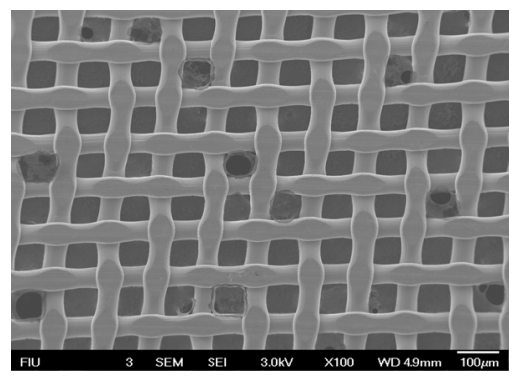

(a)

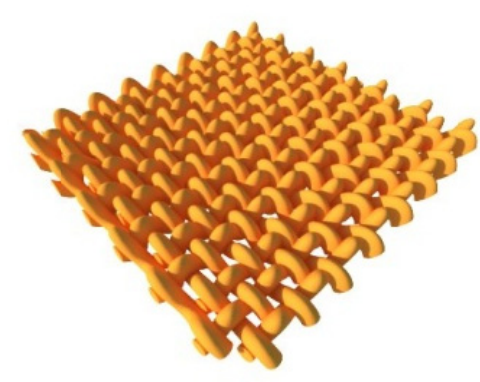

(b)

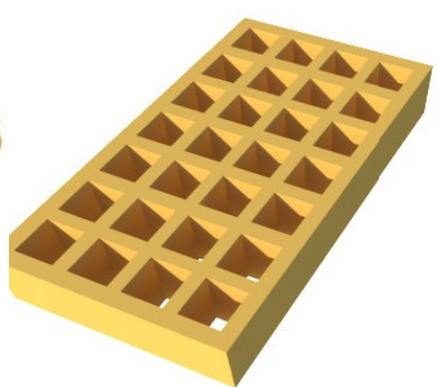

(c)

Figure 30. (a) A real SEM image of a 3D Cu mesh and the schematic diagrams (not to scale) to compare (b) the similar geometry of a 3D Cu mesh with (c) the simplified geometry of a 3D Cu mesh.

Next, determine an equivalent rectangular cuboid out of a right circular cylinder.
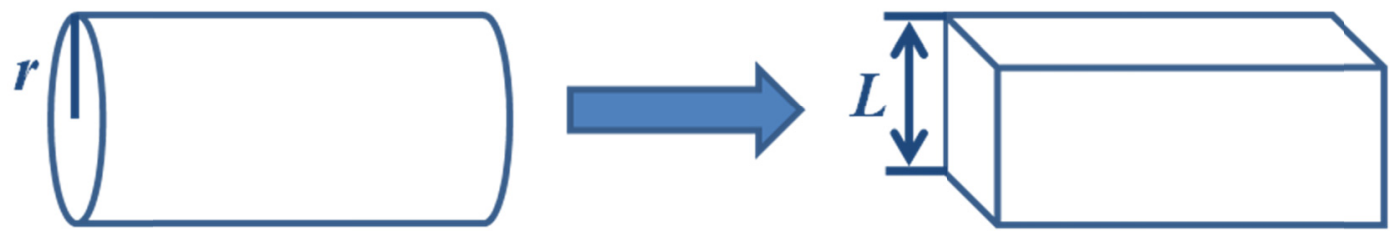

$$
\pi r^{2}=L^{2}
$$

Bare $\mathrm{Cu}$ wires (A single wire has a diameter of $50.8 \mu \mathrm{m}$.) are interlaced to form a 3D $\mathrm{Cu}$ mesh (TWP, Inc.)

Substituting $\mathrm{r}=25.4 \mu \mathrm{m}$ into the equation (1), we can obtain $\mathrm{L}=45.02 \mu \mathrm{m}$. 
Step 2.

Imagine a $\mathrm{Cu}$ mesh with 4 holes. As-purchased $\mathrm{Cu}$ mesh has a single hole area with $76 \mu \mathrm{m}$ length (TWP, Inc.).

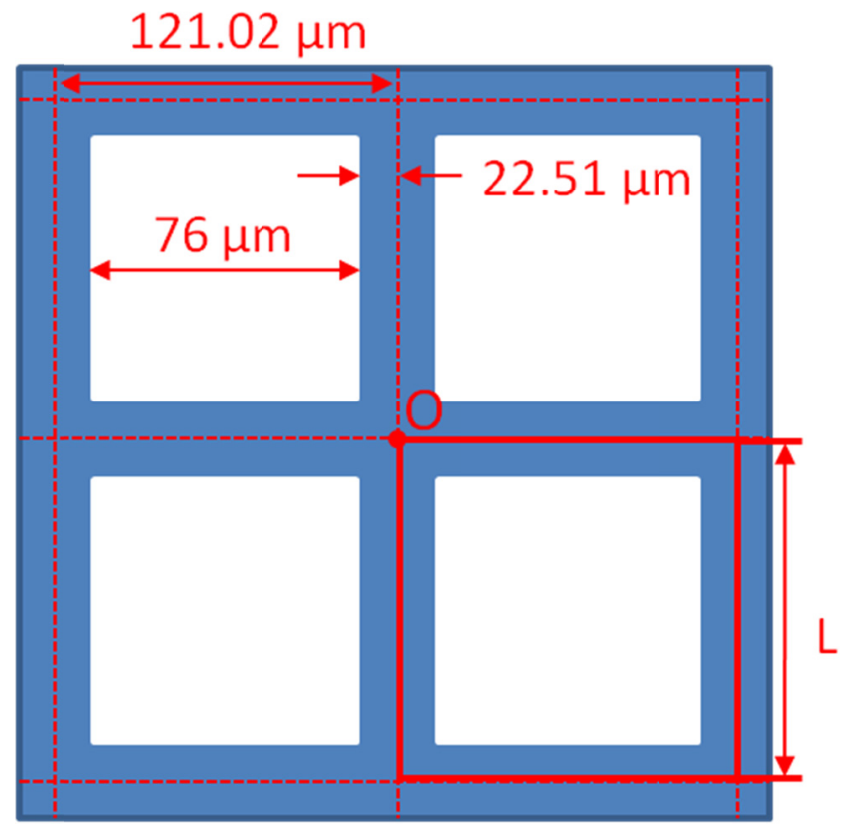

Figure 31. Schematic plane view of a 3D Cu mesh with 4 holes

Decide the point $\mathrm{O}$ as an original point and place the point at the top left corner of the $\mathrm{Cu}$ mesh with $2 \mathrm{~cm}$ by $2 \mathrm{~cm}$ area. From the arrangement, the number of arrayed unit cells with length $\mathrm{L}$ in the whole $\mathrm{Cu}$ mesh can be calculated as follows:

$$
\frac{20000}{(L+45.02)} \times \frac{20000}{(L+45.02)} \ldots \ldots(2)
$$


Step 3.

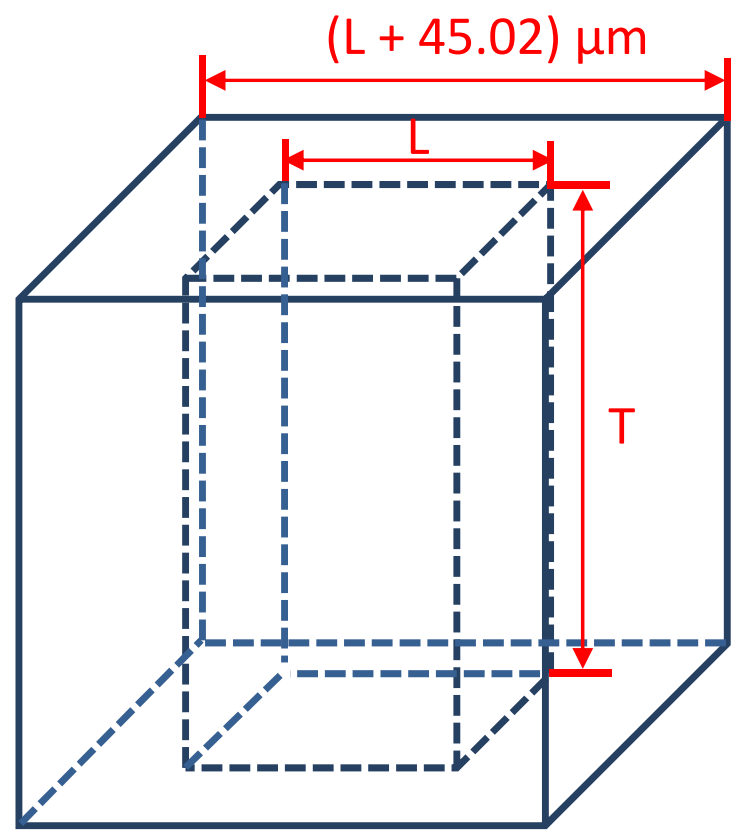

Figure 32. Schematic diagram of 3-dimensional view of a unit cell of a 3D Cu mesh

Formula for the calculation of the surface area of the unit cell has three main parts that are summed together as follows:

1. The surface area of the top planes

$$
\frac{\left[\left\{(L+45.02)^{2}-L^{2}\right\} \times 2\right] \times\left(\frac{20000}{L+45.02}\right)^{2}}{2}
$$


2. The surface area of the lateral inside planes

$$
\{(L \times T) \times 4\} \times\left(\frac{20000}{L+45.02}\right)^{2}
$$

3. The surface area of the lateral outside cells should not be considered for the calculation since they are enclosed by their neighbor cells.

The formula to calculate the total surface area of the $3 \mathrm{D} \mathrm{Cu}$ mesh is then obtained by summing overall the surface area mentioned above, as follows:

$$
\frac{\left[\left\{(L+45.02)^{2}-L^{2}\right\} \times 2\right] \times\left(\frac{20000}{L+45.02}\right)^{2}}{2}+\{(L \times T) \times 4\} \times\left(\frac{20000}{L+45.02}\right)^{2}
$$

Figure 33 shows the variation of the surface area and its ratio of the $3 \mathrm{D} \mathrm{Cu}$ mesh to the $2 \mathrm{D} \mathrm{Cu}$ foil as a function of the thickness $(\mathrm{T})$ and the size of holes (L) of the 3D Cu mesh. 


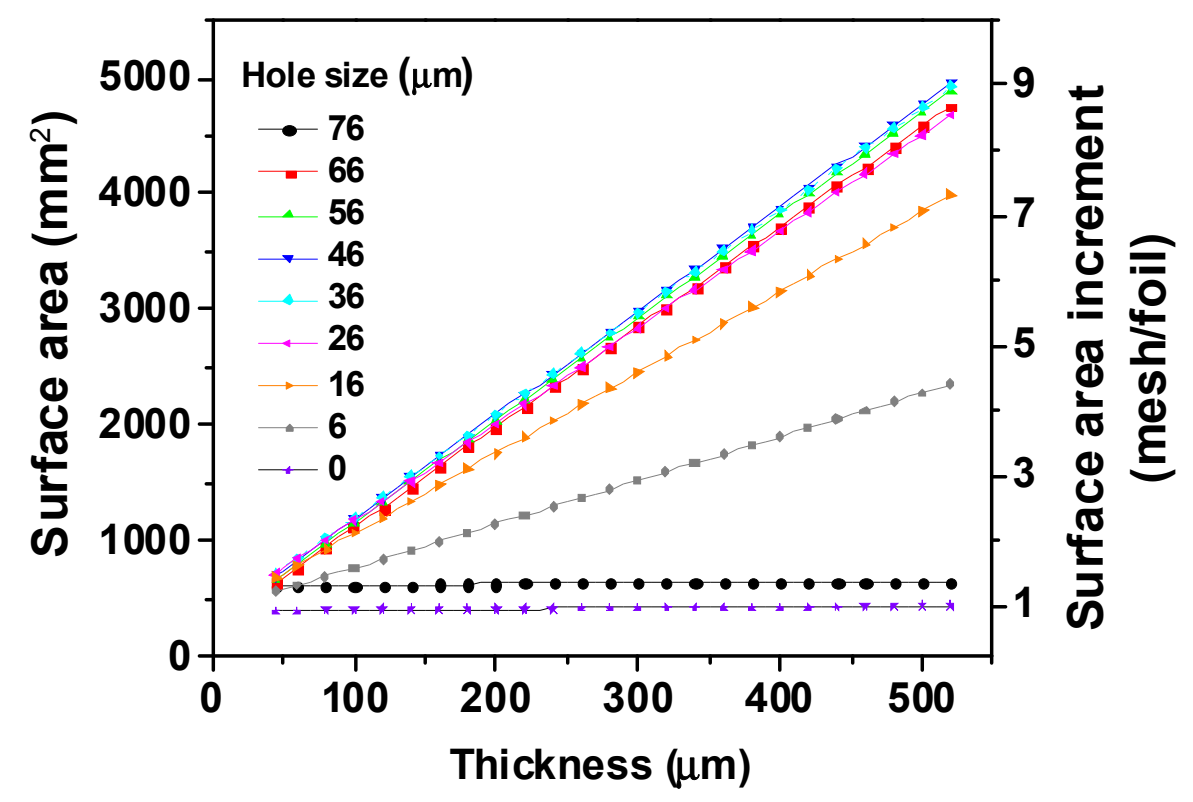

Figure 33. The surface area and its increment of the 3D Cu mesh and the $2 \mathrm{D} \mathrm{Cu}$ foil as a function of different thickness and hole sizes.

3D $\mathrm{Cu}$ mesh was an interlaced structure of numerous $\mathrm{Cu}$ wires (Figure 30(a) and Figure 30(b).). Simple geometrical model was followed to calculate the total surface area of this 3D structure, since the calculation of the actual surface area of the assembled 3D Cu mesh was very difficult. For easier calculation, the cylindrical $\mathrm{Cu}$ wires were simplified to rectangular wires of equivalent surface area, creating a cuboid array (Figure 30(c)). In this study, MWCNTs were synthesized on the top and lateral surface areas of the 3D $\mathrm{Cu}$ mesh only, hence only these areas were considered for calculation.

Figure 34(a) was a schematic view of an anode stack built up of 4 layers of MWCNTs on 3D $\mathrm{Cu}$ mesh. As a real example, we implemented an anode stack system using 9 individual MWCNTs on 3D $\mathrm{Cu}$ mesh, in which MWCNTs were coated over the entire area of the $\mathrm{Cu}$ 
meshes as illustrated in Figure 34(b). The assembled anodes were physically bonded by adhesion force between the entangled MWCNTs. This phenomenon may be thought of as being in some way analogous to "Velcro" effect of scales on wool fibers [82]. A simple geometrical relationship was established to calculate the total surface area of $3 \mathrm{D} \mathrm{Cu}$ mesh and $2 \mathrm{D} \mathrm{Cu}$ foil (no hole). Variation of the surface ratio of $3 \mathrm{D} \mathrm{Cu}$ mesh to $2 \mathrm{D} \mathrm{Cu}$ foil as a function of the thickness $(\mathrm{T})$ and the size of holes (L) of 3D Cu mesh is presented in Figure 34(c) (The detail calculation procedure is mentioned above.). Surface area increased with the thickness of the structure, though its relation with hole size was not straight forward owing to contribution from a fixed $\mathrm{Cu}$ wire diameter. It may be recalled here that the present study used a $\mathrm{Cu}$ mesh with its dimension $50 \mu \mathrm{m}$ thickness and $65 \mu \mathrm{m}$ hole size, which showed surface area improvement (almost 60\%) as compared to 2D Cu foil. Empirically, the average weight of MWCNTs on 3D Cu mesh was 4.37 mg for a cell with $14 \mathrm{~mm}$ diameter and $50 \mu \mathrm{m}$ thickness dimension, which represented solid loading increase by $400 \%$. Thus, it was found that the theoretical calculation was inconsistent with the experimental results since it did not include the influence of control parameters on the properties of MWCNTs, such as their diameters, lengths and densities during the CVD growth. From an anode system assembled using 9 individual MWCNTs on 3D $\mathrm{Cu}$ mesh (The size of a cell is $15 \mathrm{~mm}$ diameter and $50 \mu \mathrm{m}$ thickness.), the average maximum loading amount and packing density of MWCNTs were higher than $50 \mathrm{mg}$ and $0.248 \mathrm{~g} / \mathrm{cm}^{3}$, respectively. The weight of stacked anodes increased proportionally with additional anodes, which is in a good agreement with the calculation results. 


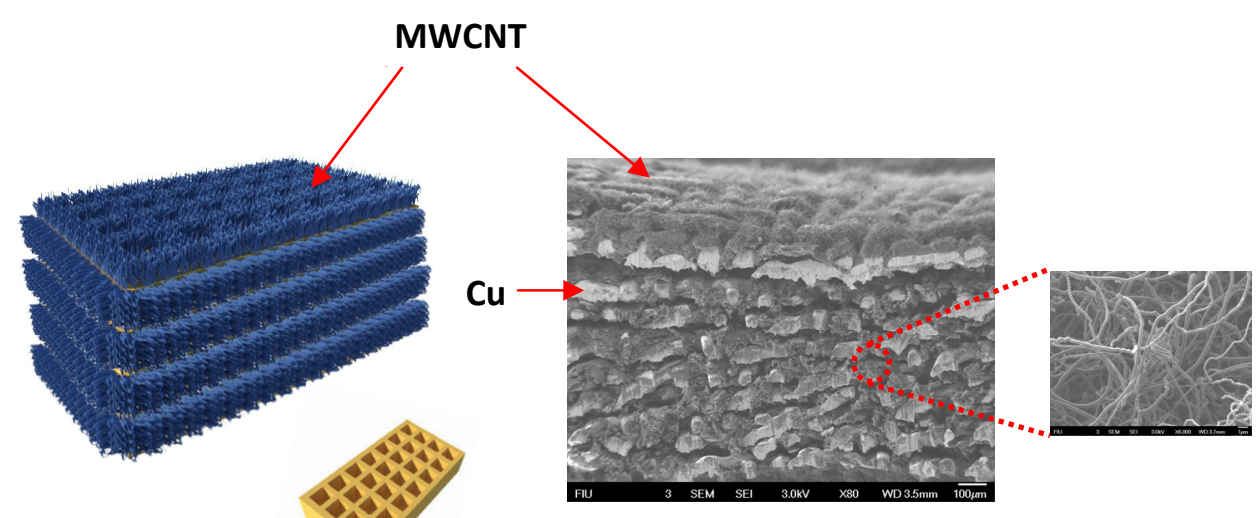

(a)

(b)

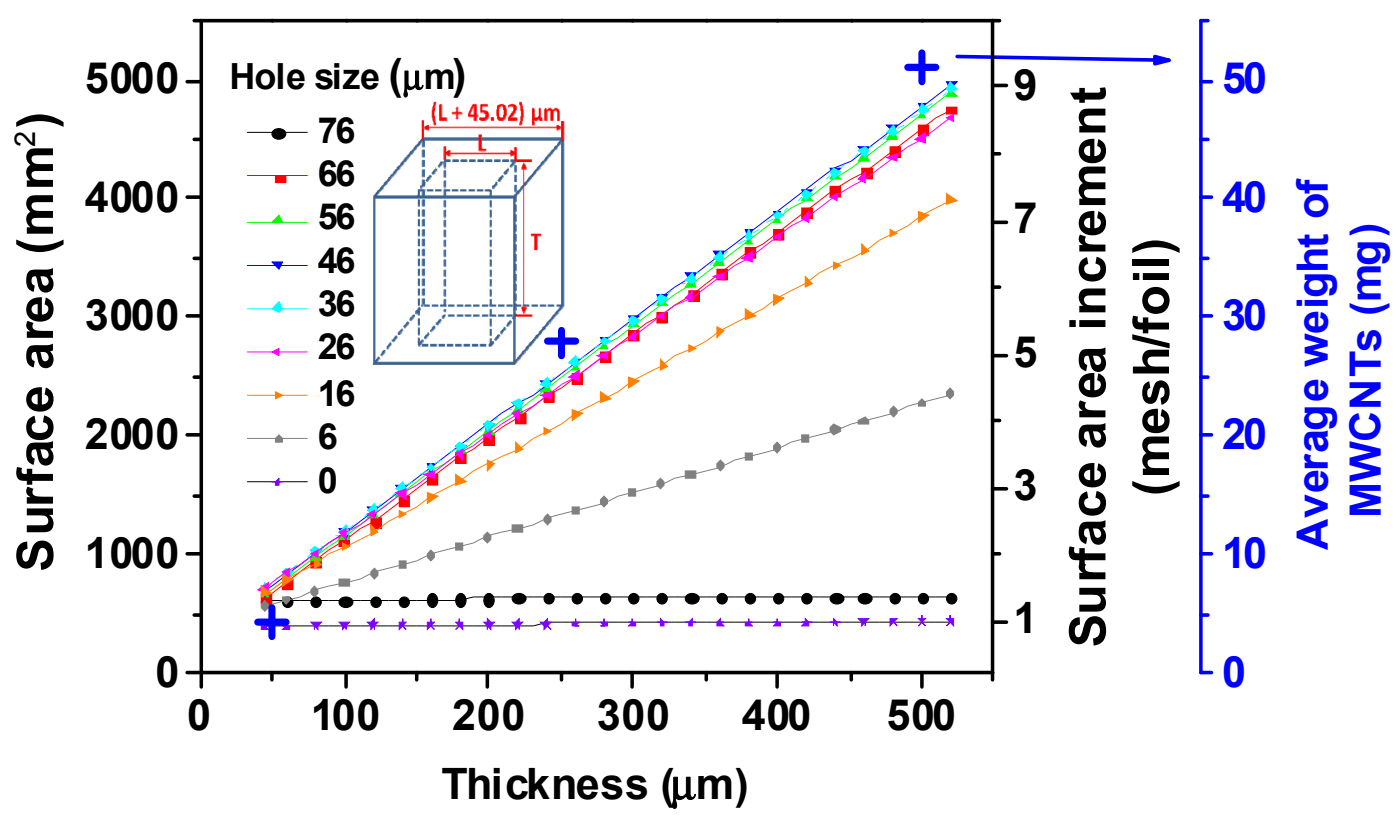

(c)

Figure 34. (a) A schematic model of an anode stack assembled using 4 numbers of converted uniform stacking cuboid arrays from the geometry of a real 3D Cu mesh (The bottom right inset shows a unit cell of the cuboid arrays.), (b) SEM images exhibiting a cross-section perpendicular to the anode system stacked by 9 individual MWCNTs on 3D $\mathrm{Cu}$ mesh and highly entangled structures of MWCNTs, (c) The surface area and its increment of the 3D Cu mesh and the 2D Cu foil and the average real weight of MWCNTs as a function of different thickness and hole sizes (The inset illustrates a unit cell of the 3D Cu mesh with its dimension.). 


\subsubsection{Morphology and Structural Properties of MWCNTs and a-Si/MWCNTs on 3D Cu}

Mesh

Unique 3D structure of the proposed anode is expected to influence the electrochemical performance. Structural characterization, involving SEM and Raman spectroscopic analysis, has been conducted on these 3D anodes (See Figure 35). As presented in Figure 35(a), MWCNTs were homogeneously and densely grown over the whole top and lateral $\mathrm{Cu}$ mesh area. In addition, the high magnification SEM image showed the randomly entangled MWCNTs with their length around $30 \mu \mathrm{m}$ and diameter in the range of $200-300 \mathrm{~nm}$ (See Figure 35(b)). Compared to the 2D $\mathrm{Cu}$ foil, the surface areas of the $\mathrm{Cu}$ wires surrounding the hole spaces could be available sites for the MWCNT growth, which is structural advantage of the 3D Cu mesh to increase the weight of the MWCNTs as active materials. In Figure 35(c), the sketch of the cross section view of MWCNTs grown on a single $\mathrm{Cu}$ wire exhibited the densely grown larger diameter MWCNTs on the top surface area and relatively smaller diameter MWCNTs on the lateral side area. Along with the structural characteristic, the interfacial control between active material and current collector significantly influences the electrochemical performance of the anode system. It is highlighted that the strong bonding between MWCNTs and Cu making it possible to directly grow MWCNTs on $\mathrm{Cu}$ mainly stemmed from $\mathrm{TiC}$ underlayer $[34,66]$. The $\mathrm{TiC}$ underlayer was formed by the reaction of $\mathrm{Ti}$ thin film sputter deposited onto 3D $\mathrm{Cu}$ mesh with carbon precursor gas at high temperature about $750{ }^{\circ} \mathrm{C}$ during $\mathrm{CVD}$ processing. High ratio of $\mathrm{I}_{\mathrm{D}} / \mathrm{I}_{\mathrm{G}}$ (around 1) in Raman spectra (Figure 35(e)) of the MWCNT structure also revealed high defect density in the structure. It is reported that the MWCNTs containing more defects showed the better electrochemical performance since the higher presence of defects provided with more available sites for $\mathrm{Li}^{+}$ion intakes into the MWCNTs structure and shortened the diffusion length of $\mathrm{Li}^{+}$ion [83-84]. MWCNTs possessing more defects, generated by mechanical breakage and chemical etching, resulted in an increase in specific capacity compared to untreated MWCNTs. In the case of 


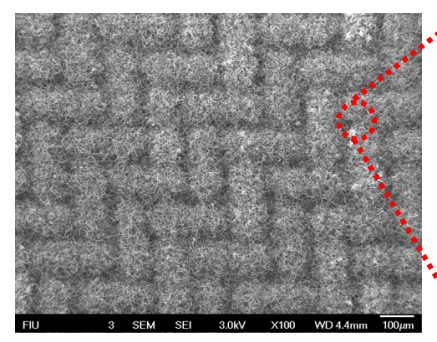

(a)

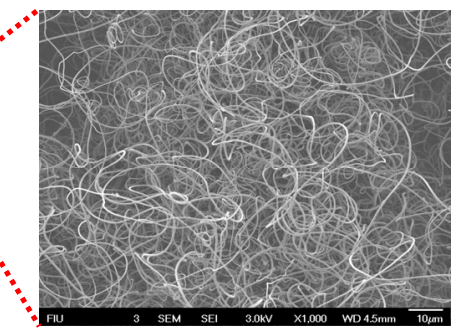

(b)
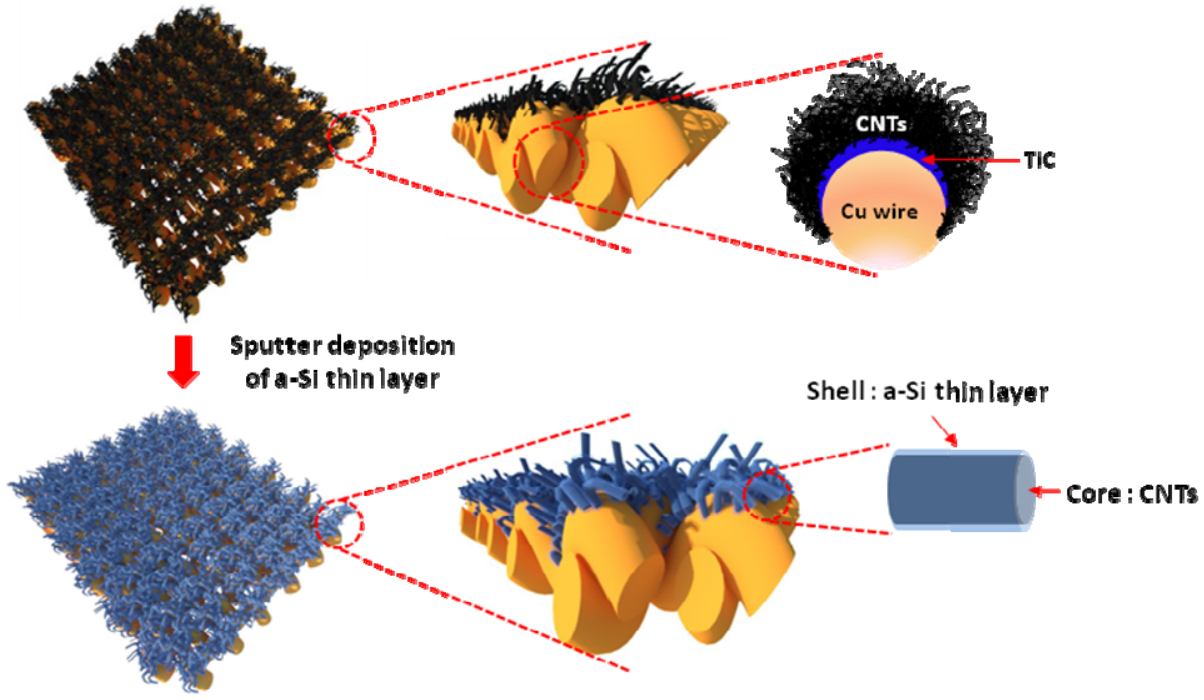

(c)

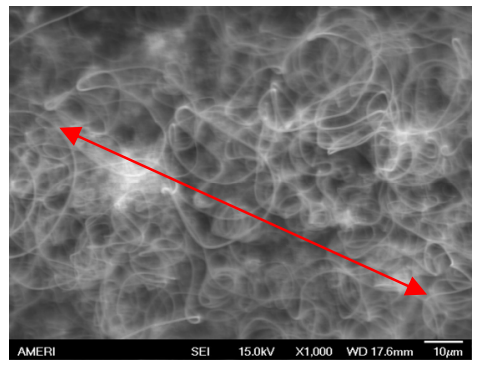

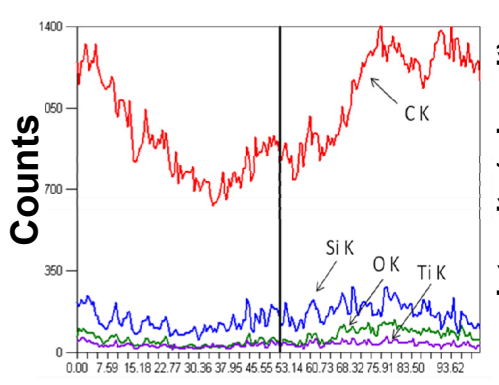

(d)

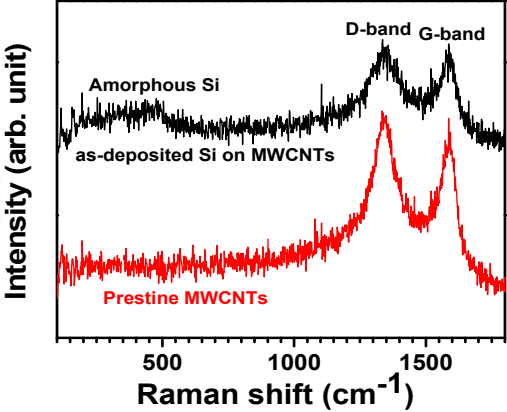

(e)

Figure 35. Morphology and structure of the proposed anode systems. (a) A plane view of SEM image showing the MWCNTs covered on the 3D Cu mesh, (b) The threadlike grass structures of MWCNTs on the 3D $\mathrm{Cu}$ mesh with their diameter in the range of $200-300 \mathrm{~nm}$, (c) Schematic diagram (not to scale) of the geometry of the MWCNTs grown on the 3D Cu mesh and the a-Si deposited MWCNTs structure on the 3D $\mathrm{Cu}$ mesh, (d) EDS elemental analysis of the a-Si/MWCNTs hybrid structure in the linearly selected area across the SEM image, (e) Raman spectroscopic response indicating high defect density of MWCNTs according to $\mathrm{I}_{\mathrm{D}} / \mathrm{I}_{\mathrm{G}}$ ratio around 1 and the amorphous Si peak at around $500 \mathrm{~cm}^{-1}$ in the a-Si/MWCNTs. 
a-Si/MWCNTs hybrid structure, the broad intensity peak around $500 \mathrm{~cm}^{-1}$ (in Raman spectra) showed amorphous $\mathrm{Si}$ [85]. It was subsequently confirmed from the EDS analysis in the linearly selected area across the SEM image as shown in Figure 35(d) that Si was deposited on some portion of the surface of the MWCNTs. The line profile denoting Si K distinguishably appeared, demonstrating the presence of a-Si deposited layer.

\subsubsection{Electrochemical Performances of the MWCNT-Based Anode Systems}

\subsubsection{Charge/Discharge Capacities}

Electrochemical performance tests were conducted on these anode structures and the results were presented in Figure 36. First charge-discharge curves for all the anode systems were presented in Figure 36(a). The button cells were charged/discharged in a galvanostatic mode between 0.01 and $3 \mathrm{~V}$, at C-rate of $0.1 \mathrm{C}$ (specific current $38 \mathrm{mAg}^{-1}$ ) determined by theoretical specific capacity of graphite $\left(372 \mathrm{mAhg}^{-1}\right)$. In the case of MWCNTs on 3D Cu mesh electrodes, several samples with different solid loading of MWCNTs were measured under the same condition and showed average discharge capacity $474 \mathrm{mAhg}^{-1}, \mathrm{LiC}_{4.7}$. In Figure 36(a), all the discharge curves exhibited a plateau in the voltage range $0.75-0.9 \mathrm{~V}$. Such plateau was found in most graphite or CNT-based anodes and stemmed from the decomposition of electrolyte and the formation of solid electrolyte interphase (SEI) on the carbonaceous anode materials $[14,86]$.

Recently, D. Aurbach reviewed extensive studies on the composition of the SEI layer formed on lithiated carbon and lithiated transition metal oxide electrodes in a wide variety of organic electrolyte solutions [55]. These studies employed spectroscopic measurements (Fourier Transform Infrared Spectroscopy (FTIR), X-ray Photoelectron Spectroscopy (XPS), Energy Dispersive X-ray Analysis (EDAX)). In our study, a fluorine-rich region in the SEI layer was 


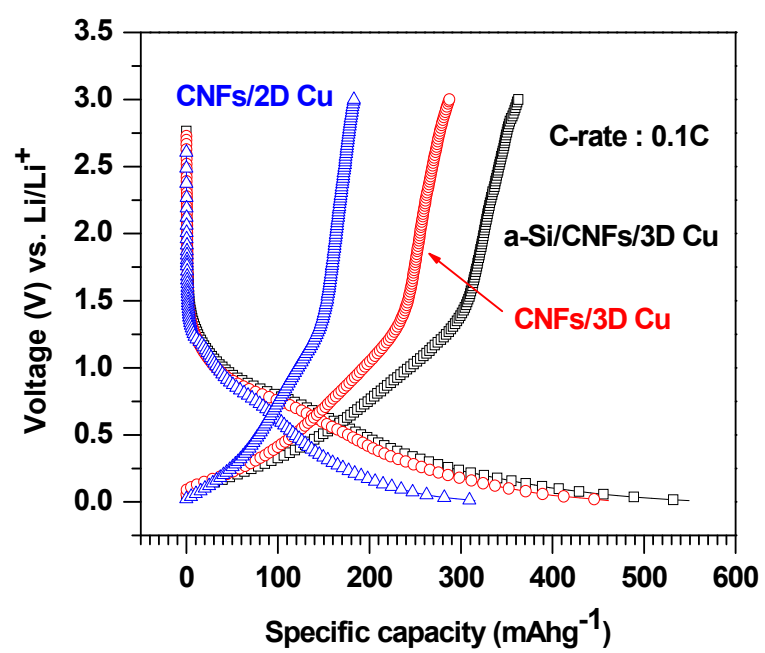

(a)

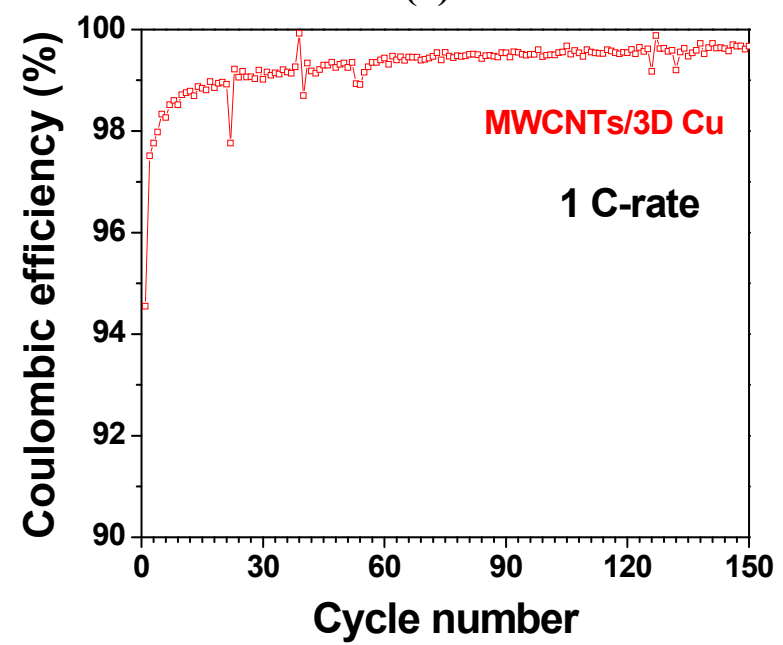

(c)

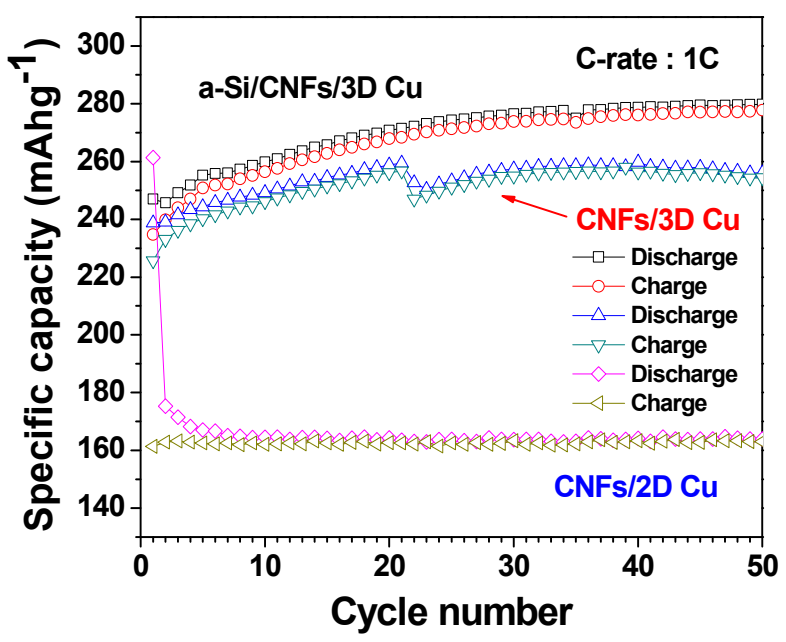

(b)

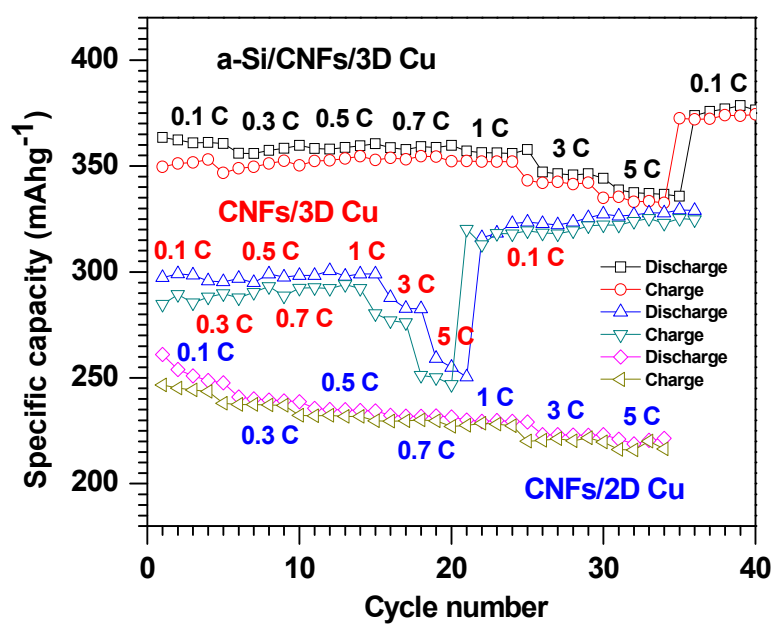

(d)

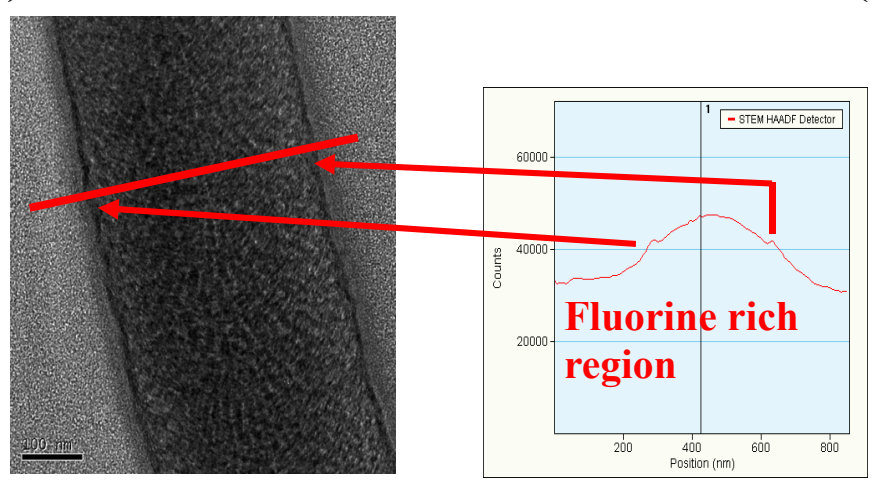

(e)

Figure 36. Electrochemical performance of the anode structures of as-grown MWCNTs on 3D Cu mesh, MWCNTs on 2D Cu foil and a-Si/MWCNTs core-shell composite on 3D Cu mesh. Part (a); first charge-discharge cycle at $0.1 \mathrm{C}$-rate, (b) comparison of the cycling stability of the different 
anode structures, (c) Coulombic efficiency at 1 C-rate; (d) the variation of reversible capacities at different C-rates; (e) Scanning Transmission Electron Microscopy (STEM) and High-Angle Annular Dark Field (HAADF) cross-sectional view of a single MWCNT and the intensity profile computed along the red line displayed in the cross-sectional view.

discovered by using a Scanning Transmission Electron Microscopy (STEM) and High-Angle Annular Dark Field (HAADF) cross-sectional view of a single MWCNT, and the intensity profile computed along the red line is displayed in the image (see Figure 36(e)). We assumed that the fluorine rich region was created by the following reaction scheme:

$$
\begin{gathered}
\mathrm{LiPF}_{6} \cdot \mathrm{LiF}+P F_{5} \\
\mathrm{PF}_{5}^{-}+n e^{-}+n L i^{+} \rightarrow L i F \downarrow+L i_{x} P F_{y} \downarrow \\
P F_{3} \mathrm{O}+n e^{-}+n L i^{+} \rightarrow L i F \downarrow+L i_{x} P O F_{y} \downarrow
\end{gathered}
$$

Note that equations (6) - (8) do not offer the exact stoichiometry, but instead offer the basic reactions. However, a phosphorus-rich region was not identified from the STEM-HAADF technique.

Based on the similar first discharge curves of the MWCNT and the a-Si/MWCNT electrodes, it was implied that the MWCNTs mainly controlled the electrochemical properties in both anode structures since the major weight (above 99\% weight ratio) of the structure came from the MWCNTs. However, the effects of a-Si incorporation on the electrochemical performance were investigated by the first charge curves of the anodes. The average specific charge capacities of the MWCNT and the a-Si/MWCNT electrodes were $299 \mathrm{mAhg}^{-1}$ and $345 \mathrm{mAhg}^{-1}$ (almost 16\% 
improvement), respectively, which were obtained from three different batches of each of the LIB cells comprising the three different anode systems. In addition, the Coulombic efficiency of the aSi/MWCNTs was $67 \%$, which was approximately $4 \%$ higher than that of the MWCNTs. The relatively higher irreversible capacity at the first charge-discharge cycle is considered a critical limitation of carbonaceous anode materials, and the capacity loss may be mainly associated with solid electrolyte interphase (SEI) formation or a permanent alloy formation [86]. Another possible reason involved the insertion of lithium ions into defect sites (e.g., microcavities) residing in the MWCNTs and their entangled structures [87]. Lithium ions inserted into the defect regions at the first discharge process might be trapped, and it might not be completely extracted during the charge process. Lithium oxide formation at higher voltage could also be another reason for the initial capacity loss [26]. However, Figure 36(a) showed that most Li insertion processes proceeded below $1.5 \mathrm{~V}$ (versus $\mathrm{Li} / \mathrm{Li}^{+}$) so that the possibility of charge consumption due to lithium oxide formation may be excluded, in the present case. Higher values from the a-Si/MWCNTs indicated the a-Si thin layer could play a role in maintaining the stability of SEI formation [35]. On the contrary, the first discharge curves of all the specimens of the MWCNTs on 2D Cu foil represented the average specific discharge capacity $323 \mathrm{mAhg}^{-1}, \mathrm{LiC}_{6.9}$. The average capacity value was more than three times lower than that of our previous result [34]. It was speculated that the significant difference was due to the different morphology of MWCNTs (average diameter 80 $\mathrm{nm}$, which was thinner than that of the current MWCNTs). At higher growth temperatures, the size of the catalytic Ni islands became larger on account of their more facile diffusion [88]. With respect to major electrochemically active sites being on and near the surface of MWCNTs [34], the thicker MWCNTs have less active surface area per mass available for participating in electrochemical reactions as compared to the thinner MWCNTs, thereby resulting in the lower specific capacity. During the initial cycle, the MWCNTs on 3D Cu mesh offered a $47 \%$ higher average specific capacity compared to that of the MWCNTs on 2D Cu foil. It may be noted here 
that this specific capacity enhancement is close to $60 \%$ of the surface area enhancement. Compared to the MWCNTs on 3D Cu anodes, the a-Si deposited MWCNT anodes offered the higher average specific discharge capacity $517 \mathrm{mAhg}^{-1}, \mathrm{LiC}_{4.3}$. This enhancement could be related to the presence of a-Si thin layer with its high specific capacity on the MWCNTs. It was observed that the weight of the deposited a-Si was lower than $1 \%$ of that of the MWCNTs; therefore, the aSi thin layer did not have any major contribution except slight increase in the overall specific capacity of the anode, unlike the previous results from Si nanotubes [15] and Si nanowires [16].

\subsubsection{Cycling Stability}

Cycling stability tests are essential to prove electrochemical performance of the anodes at long cycling operations in the real application. Figure 36(b) illustrates a comparison of the cycling stabilities obtained from the three different kinds of anode systems up to 50 cycles at $1 \mathrm{C}$-rate (current density $372 \mathrm{mAg}^{-1}$ ). Although the cycling performances were conducted by single measurements of each of the three different anode systems, they could well describe the characterization of all three batch samples in each group of anodes. The primary rationale behind the hypothesis may be mentioned as follows: an LIB anode cell, exhibiting a good electrochemical performance attained from a primary charge-discharge cycle, generally shows good LIB performances in terms of a cycling stability and a C-rate capability over the prolonged cycles. In the case of the MWCNTs on 3D Cu mesh electrode, the reversible capacity slightly increased from $226 \mathrm{mAhg}^{-1}$ at the first cycle to $258 \mathrm{mAhg}^{-1}$ at the 39 th cycle and then gradually faded to $254 \mathrm{mAhg}^{-1}$ until the 50th cycle. Such a trend of increase in capacity over the whole cycles is inconsistent with previous results from the constant capacity of MWCNTs [34-35]. It was speculated that the continuous charge-discharge process led to the creation of more surface area on the densely entangled MWCNT network structure. We also investigated whether such an 
activation process was due to more defects in the structures of MWCNTs induced by the chargedischarge process.

According to the SEM images presented in Figure 37(a) and (b), it was not evident that severe defects or structural damages appeared in the 150 cycled anode system and just one discharged anode system. HRTEM images revealed that for both anode systems, the structural integrity of graphene layers still remained with the shallow amorphous second phase region on the edge of the MWCNTs, as shown in Figure 37(c) and (d). In addition, it was pointed out that there was no appreciable volume variation during lithiation and delithiation. No clear evidence of diameter change was observed between the as-grown MWCNTs and the MWCNTs subjected to 150 charge-discharge cycles (Figure 35(b) and 37(a)). This observation is in agreement with small volume changes of graphitic carbon upon lithium insertion and extraction (typically $\mathrm{LiC}_{6}$, around $12 \%)$ [89]. In the same manner, it was confirmed that the specific capacity of the a-Si/MWCNTs on $3 \mathrm{D} \mathrm{Cu}$ was gradually increased.

Along with the electrochemical activation process of MWCNTs, the result could reveal the effect of capacity improvement with additionally incorporated a-Si to take more $\mathrm{Li}^{+}$ion intakes (almost $10 \%$ enhancement of the reversible capacity). Furthermore, the $\mathrm{Si}-\mathrm{C}$ bonding at the interface between a-Si and MWCNTs was strong enough to avoid peeling off of the a-Si layer during the cycling test. It was highlighted that MWCNTs and a-Si/MWCNTs grown on 3D $\mathrm{Cu}$ mesh showed good cycling stability without severe capacity degradation. However, for the MWCNTs on 2D Cu foil, the average reversible capacity $163 \mathrm{mAhg}^{-1}$ was kept constant until the number of 50 cycles. It should be noted that the larger reversible capacity of the 3D MWCNTs was presumably due to their large surface area to accommodate more $\mathrm{Li}^{+}$ion intercalation.

Nevertheless, we found that some kinks appeared at the cycle No. 1, 21, 38 and 39. The reasons for the kinks at each point can be explained as follows: in the case of No. 1, since the 
anode is composed of 3D MWCNTs, the initial lithium insertions are taking place on the top surface of MWCNTs, but later cycles undergo deeper lithium insertions; hence, the capacity is increasing during the later cycles. In the case of No. 21, the actual reason for the kink is because the person, who took measurements, stopped after 20 cycles, and then, he continued the measurements from the 21st cycle. Once he stopped the measurements, the cell came to the chemical equilibrium stage; therefore, when he restarted the measurements, the cell did not start as before, but rather, it started at a low voltage. In the case of No. 38, the lithium insertion might have been hindered by SEI layers so that the capacity slightly decreased. Due to the decreased capacity, lithium ions might have again found free-sites to be inserted, resulting in the increase in capacity. The phenomenon solely stemmed from stabilization/finding deeper sites due to the 3D structure of our anode systems. In the case of No. 39, owing to new sites for lithium insertion into the $3 \mathrm{D}$ structure, capacity was slightly high, and then the capacity decreased due to chemical instability.

In terms of the reversibility of charge-discharge process, the Coulombic efficiency of the MWCNTs on 3D $\mathrm{Cu}$ mesh was more than $98 \%$ after the initial 4 cycles at $1.0 \mathrm{C}$-rate and then increased to $99 \%$ after 22 cycles according to the result shown in Figure 36(c). Such reversibility was ascribed to the sufficient supply of $\mathrm{Li}^{+}$ions from Li metal foil used as the reference electrode and counter electrode in the button cell. 


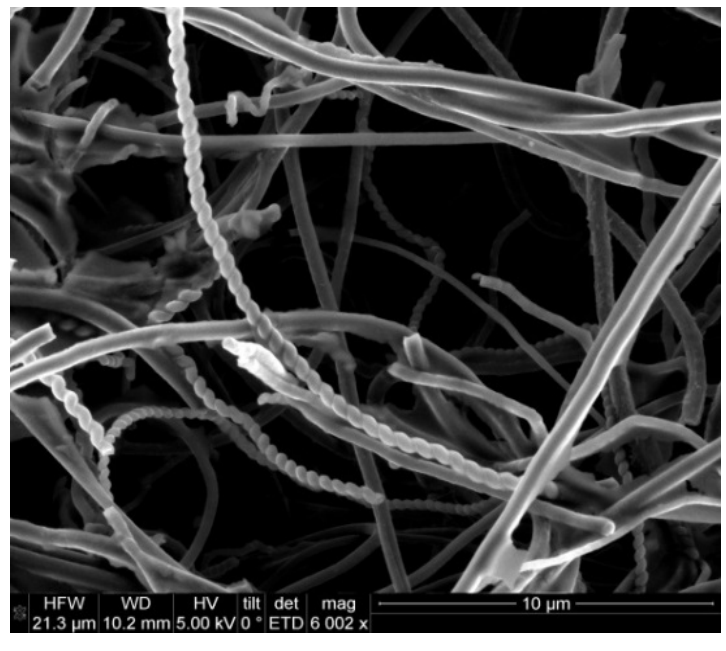

(a)

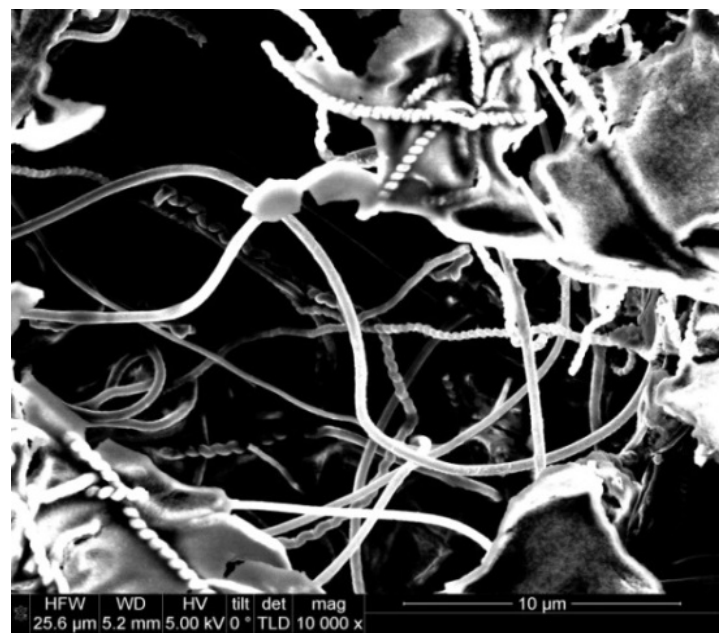

(b)

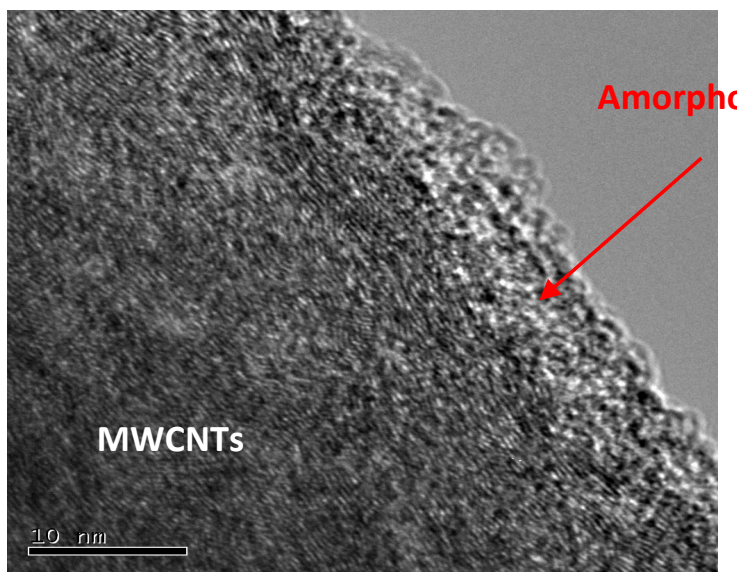

(c)

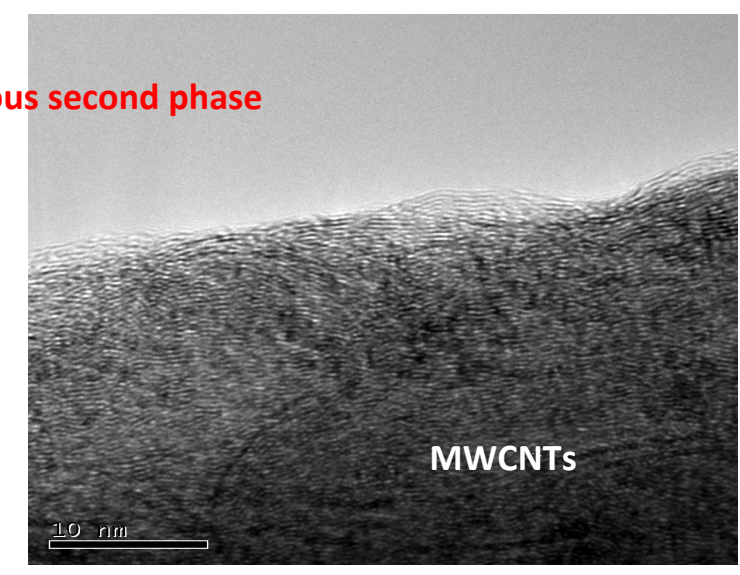

(d)

Figure 37. Plane view SEM images of (a) the MWCNTs on 3D $\mathrm{Cu}$ mesh anodes after 150 cycling at 1 C-rate and (b) the MWCNTs after only 1 discharge cycling at the same C-rate. HRTEM images of (c) the 150 cycled one and (d) the 1 discharge cycled one. 


\subsubsection{Rate Capability}

Rate capability tests are also crucial to prove electrochemical performance of the anodes at higher charge-discharge rate operations. Figure 36(d) showed the rate capability of the proposed anodes. The discharge capacity of the MWCNTs on 3D Cu mesh was almost constant around 300 $\mathrm{mAhg}^{-1}$ until reaching $1 \mathrm{C}$-rate $\left(372 \mathrm{mAg}^{-1}\right)$, and then, decreased to $283 \mathrm{mAhg}^{-1}$ at $3 \mathrm{C}$-rate $(1,116$ $\left.\mathrm{mAg}^{-1}\right)$ and further to $250 \mathrm{mAhg}^{-1}$ at $5 \mathrm{C}$-rate $\left(1,860 \mathrm{mAg}^{-1}\right)$. It may be highlighted that discharge capacity slightly increased with $\mathrm{C}$-rate from $0.1 \mathrm{C}$ to $1 \mathrm{C}$ and the phenomenon could be attributed to the electrochemical activation process of MWCNTs during cycling as mentioned above. At higher C-rates (e.g., $3 \mathrm{C}$ and $5 \mathrm{C}$ ), $\mathrm{Li}^{+}$ions were inserted and extracted into and from the only surface regions of the forest-like MWCNT structure due to the limitation of diffusion length of $\mathrm{Li}^{+}$ions. Another interesting observation was slight increase in specific capacity from $283 \mathrm{mAhg}{ }^{-}$

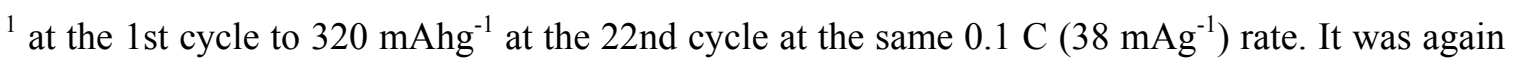
confirmed that higher number of cycles during C-rate tests could be a reason to effectively carry out electrochemical activation process in the MWCNT structures. On the contrary, the MWCNTs on $2 \mathrm{D} \mathrm{Cu}$ foil electrode showed a typical staircase type decreasing capacity behavior till the tested limit of $5 \mathrm{C}$-rate. The direct comparison revealed the higher C-rate capability of the MWCNTs on 3D Cu mesh electrode, as compared to the MWCNTs on 2D Cu foil. In particular, at $5 \mathrm{C}$, the average specific discharge capacity of the MWCNTs on 3D Cu mesh was $249 \mathrm{mAhg}^{-1}$ (14\% increase), whereas that of the MWCNTs on 2D Cu foil was $218 \mathrm{mAhg}^{-1}$. The result could be strongly associated with the higher surface area for $\mathrm{Li}^{+}$ion intercalation due to approximately four times higher solid loading of MWCNTs on 3D $\mathrm{Cu}$ mesh. Furthermore, the higher C-rate capability is significantly attributed to the strong bonding and the lower electric resistance between $\mathrm{Cu}$ and MWCNTs, through TiC interfacial layer $[69,72]$. Moreover, at $5 \mathrm{C}$, the average capacity of the a-Si/MWCNTs on 3D Cu mesh electrode was $334 \mathrm{mAhg}^{-1}$ (34 \% enhancement), while that of the MWCNTs on 3D Cu mesh was $249 \mathrm{mAhg}^{-1}$. In addition, the average capacity 
ratios at $5 \mathrm{C} / 0.1 \mathrm{C}$ of the MWCNTs on $3 \mathrm{D} \mathrm{Cu}$ mesh and the MWCNTs on $2 \mathrm{D} \mathrm{Cu}$ foil were 0.86 and 0.8 , respectively, whereas the ratio of the a-Si/MWCNTs on 3D $\mathrm{Cu}$ mesh was around 0.93 . According to the better performances, it was proved that a-Si thin layer coating on MWCNTs could play an important role in the enhancement of C-rate capability.

\subsection{Conclusion}

A novel 3D MWCNTs on Cu current collectors as an anode of LIB was developed. The 3D anodes showed higher specific capacity, cycling stability and C-rate efficiency, as compared to those of the MWCNTs on 2D Cu foil anodes. The better performances of the 3D anodes were attributed to higher average solid loading of MWCNTs, which was four times higher than that of the $2 \mathrm{D}$ anodes. The addition of the a-Si onto the 3D MWCNTs/Cu showed the further enhancement of electrochemical properties. 


\section{CHAPTER 5.}

\section{D ANODE STACK ASSEMBLED BY MULTIWALL CARBON NANOTUBES (MWCNTS) FOR LI-ION BATTERY ANODE}

\subsection{Introduction}

It was very recently argued whether most carbon nanomaterial-based LIB anodes would or would not be practically available for their next generation EV application [36]. One of the reasons behind that could be relevant to their lower volumetric specific energy and power densities regardless of their higher gravimetric energy and power densities. To counter the issue, we would like to propose a similar novel approach, which is stacking of a number of individual free-standing 3D MWCNT mesh structures suitable for remarkable increase in solid loading of MWCNTs as active materials. The stacking method is simply placing one free-standing 3D MWCNT mesh structure formed by chemically etching away 3D Cu mesh on top of another, one by one, resulting in an increase of active 3D MWCNT materials' weight and volume in the anode stacks. The 3D free-standing MWCNT mesh structures contained approximately 35\% of void area that inhibit crack propagation, which can otherwise induce severe mechanical fracture in the structures. Thus, structural integrity of the 3D free-standing MWCNT mesh structures could remain while one handles them during their etching process from $\mathrm{Cu}$ mesh or stacking of the 3D free-standing MWCNT mesh structures by pressing them. The thread-like MWCNTs are randomly connected, enhancing the self-adhesion of a whole anode for the higher electric conductivity and robust structural integrity in the overall anode stacks. Furthermore, to enhance the bonding strength of individual MWCNT layers and their volumetric mass loading density, we use a polyvinylidene fluoride (PVDF)-based binder solution. 
In this chapter, we would like to present the unique preparation methods of the anode stack by using free-standing MWCNTs and their structural properties through some essential analytical techniques, including scanning electron microscopy (SEM), electron dispersion spectroscopy (EDS) and Raman spectroscopy. In addition, Li-ion coin cells have been assembled by using the multi-stacked MWCNT anodes and their electrochemical performances have been discussed with respect to loading amount. Furthermore, we propose a novel anode stack prepared by using the binder solution and pressing at high pressure and temperature to enhance the LIB performance and deal with the correlation between the structural and electrochemical properties of the new proposed anode stack.

\subsection{Results and Discussion}

\subsubsection{Experimental Details}

MWCNT stacks composed of multiple layers of 3D free-standing MWCNT mesh structures have been prepared with a combination of chemical vapor deposition (CVD) techniques through a two-step approach and formation of 3D free-standing MWCNT meshes after etching process of $\mathrm{Cu}$ mesh $(50 \mu \mathrm{m}$ thickness and $65 \mu \mathrm{m}$ hole size, TWP Inc.) substrates, as shown in Figure 38(a). Samples of 3D MWCNTs on $\mathrm{Cu}$ mesh were first synthesized by deposition of Ti as barrier layer to carbon diffusion into $\mathrm{Cu}$; $\mathrm{Ni}$ as catalytic thin layer for MWCNT growth onto a $\mathrm{Cu}$ mesh using an RF and DC magnetron sputtering system, and subsequently followed by the CVD growth of MWCNTs. In order to prepare CVD grown 3D MWCNTs on $\mathrm{Cu}$ mesh, the Ti/Ni sputter deposited $\mathrm{Cu}$ meshes were placed in a tubular furnace and rapidly heated to $750{ }^{\circ} \mathrm{C}$ in argon flow. And then, at $750{ }^{\circ} \mathrm{C}$, a mixture of $\mathrm{H}_{2}$ and $\mathrm{C}_{2} \mathrm{H}_{4}\left(1: 2\right.$ volume ratio of $\mathrm{H}_{2}$ to $\left.\mathrm{C}_{2} \mathrm{H}_{4}\right)$ was flowed into

the furnace for 50 minutes. Finally, the furnace was cooled down to room temperature in an $\mathrm{Ar}$ 
environment. Weights of each sample before and after CVD growth were measured for net weight of MWCNTs as active materials being applied to LIB performance tests. After $4 \sim 5$ hours of $\mathrm{Cu}$ etching process, $\mathrm{Cu}$ mesh was completely dissolved into the concentrated ferric chloride $\left(\mathrm{FeCl}_{3}\right)$ solution (Transene Company, Inc.), and the solution left 3D free-standing MWCNT mesh structures. One of the reactions for etching of copper with ferric chloride is as follows [90]:

$$
\mathrm{Cu}_{(\mathrm{S})}+2 \mathrm{Fe}^{3+}+3 \mathrm{Cl}^{-} \rightarrow \mathrm{CuCl}_{3}^{-}+2 \mathrm{Fe}^{2+}
$$

The 3D free-standing MWCNTs were thoroughly washed with de-ionized water and dried in vacuum oven at $120{ }^{\circ} \mathrm{C}$ for at least 5 hours. 5 to 8 individual 3D free-standing MWCNT meshes were placed one by one and pressed using a precision disc cutter (MTI Corp.), resulting in a whole structure of 3D MWCNT-based anode stack with its dimension of $14 \mathrm{~mm}$ diameter disk shape. A Cu mesh current collector was placed at the bottom side of the MWCNT stack for its efficient electron charge transfer. Furthermore, to prepare a highly dense anode stack, we introduced a binder solution composed of Polyvinylidene fluoride (PVDF, Sigma-Aldrich) and carbon nanoparticles (Sigma-Aldrich) (7 : 3 weight ratio) dissolved into N Methyl 2-pyrrolidone (NMP, Sigma-Aldrich) solvent. The binder solution was dropped and spread onto each freestanding MWCNT layer one by one, consequently forming five layered anode stacks. The asprepared anode stack samples were dried under the step-up temperature profile in vacuum oven at $50{ }^{\circ} \mathrm{C}$ for 1 hour, $60{ }^{\circ} \mathrm{C}$ for 40 minutes, and $80{ }^{\circ} \mathrm{C}$ for 3 hours. The relative lower drying temperatures were selected to avoid any oxidation of the $\mathrm{Cu}$ current collector placed at the bottom side of the samples. The subsequent compression of the samples created anode stacks with the areal mass density of $9 \mathrm{mg} / \mathrm{cm}^{2}$ and the volumetric mass density of $1.25 \mathrm{~g} / \mathrm{cm}^{3}$.

The surface morphology of as-grown MWCNTs was observed or measured using a field emission scanning electron microscope (FESEM) (JEOL, JSM-7000F), an energy dispersive 
spectroscope (EDS) (Thermo Electron Corporation, NORAN System SIX), a Raman spectrometer $\left(\mathrm{Ar}^{+}\right.$laser with $\lambda=514 \mathrm{~nm}, 33 \mathrm{~mW}$ power) and a field emission transmission electron microscope (FETEM) (FEI, TECHNAI F20). For the electrochemical performance tests, a coin type (CR 2032) of half-cell assembly was carried out in an argon filled glovebox under

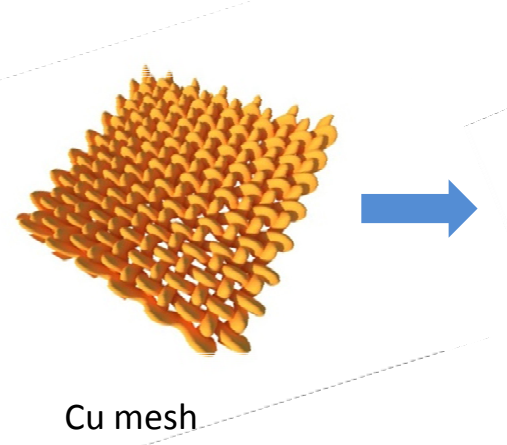

(a)

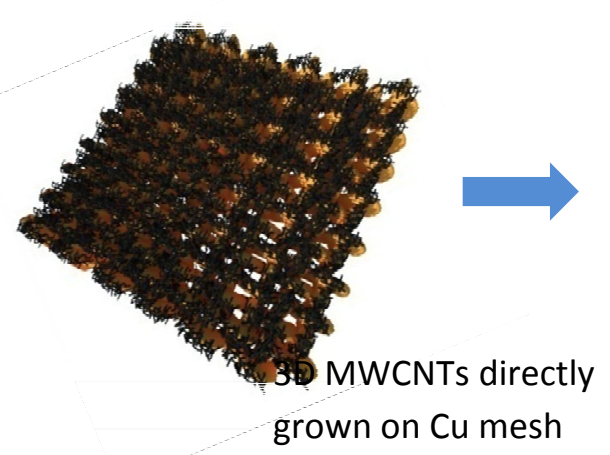

(b)

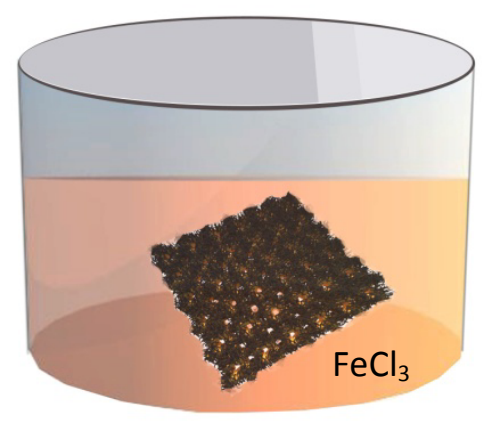

Cu etching

(c)

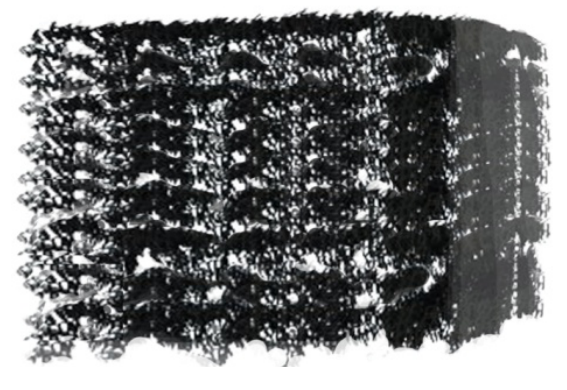

3D MWCNT stack

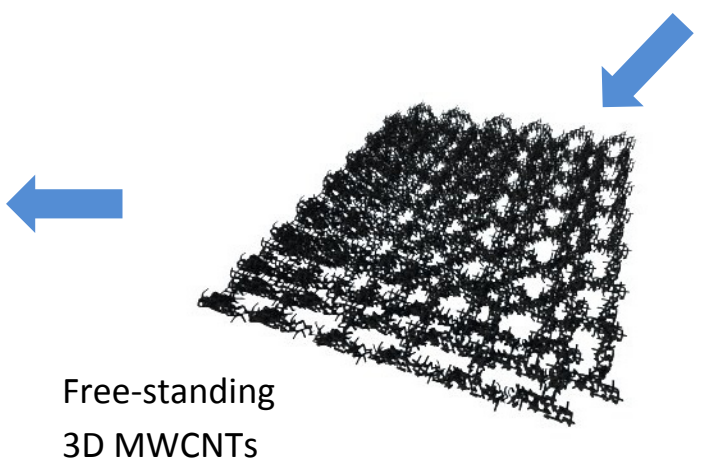

(d)

(e)

Figure 38. Process flow for anode stack system from an individual 3D MWCNTs on Cu mesh current collector to 3D free-standing MWCNT anode stack system. (a) As-received commercially available 3D $\mathrm{Cu}$ mesh, (b) CVD grown 3D MWCNTs on the surface of the $\mathrm{Cu}$ mesh, (c) Chemical etching process of the $\mathrm{Cu}$ mesh via $\mathrm{FeCl}_{3}$ etching solution, (d) $3 \mathrm{D}$ free-standing 
MWCNT mesh structure after the etching process, (e) Fabrication of anode stack consisted of multiple layers of 3D free-standing MWCNT meshes by finally pressing them together.

extremely low level of humidity $(<1 \mathrm{ppm})$. The MWCNT stack was used as working electrode, whereas Li metal foil as both counter and reference electrodes. $1 \mathrm{M}$ solution of $\mathrm{LiPF}_{6}$ salt in 1:1 $(\mathrm{v} / \mathrm{v})$ mixture solvent of ethylene carbonate (EC) and dimethylene carbonate (DMC) was used as

electrolyte. Typical polypropylene (PP) material (Separator 2400) was used as separator. The cell was finally assembled by using CR 2032 coin-cell crimping tool (Honsen Corp.). The charge and discharge tests of the assembled cells were performed in a multi-channel battery testing unit (MACCOR) at a room temperature in galvanostatic mode (constant current density). The cells were cycled in the voltage range 3.0 to $0.01 \mathrm{~V}$, recording a current value in each $10 \mathrm{mV}$ step, at a slower C-rate (e.g., 0.05C) during the initial formation process and at a faster rate (e.g., 0.5C) in the following cycles.

\subsubsection{Morphology and Structural Properties of MWCNT-Based Anode Stack}

Figure 39 shows surface morphology, TEM, EDS and Raman spectra of free-standing 3D MWCNT mesh. For the free-standing 3D MWCNTs as shown in Figure 39(a), it was very difficult to measure the overall length of MWCNTs due to their entangled structure, and the asprepared MWCNTs had an outer diameter about $300 \mathrm{~nm}$ (see Figure 39(a) inset). As seen in the HRTEM image of Figure 39(b), the internal structure of MWCNTs comprised disorder graphitic (turbostratic) stacking. Figure 39(c) represents the spot selected for EDS element analysis in order to verify whether the $\mathrm{Cu}$ was completely etched away, and the spot was intentionally chosen at the bottom side of the free-standing MWCNT structure. As seen in Figure 39(d), the EDS spectrum related to carbon appeared quite strong (higher than $93 \mathrm{wt} \%$ ), despite little of the $\mathrm{Cu}$ 
(lower than $3 \mathrm{wt} \%$ ) remained. The $2.2 \mathrm{wt} \% \mathrm{Fe}$ was due to the residue of Fe element or compound after the $\mathrm{Cu}$ etching process by an aqueous $\mathrm{FeCl}_{3}$ etching solution. However, we would like to argue that Fe can be negligible in LIB performance based on the previous report by Poizot et al. [18]. They found that Fe as one of the transitional metals did not act as Fe-Li intercalating compound. Instead, they proposed a new mechanism of Li reactivity with the transitional metals which involves the formation and decomposition of $\mathrm{Li}_{2} \mathrm{O}$, accompanying the reduction and oxidation of metal nanoparticles (in the range of $1 \pm 5 \mathrm{~nm}$ ). In addition, $1.1 \mathrm{wt} \%$ Ti comes from a barrier layer deposited on $\mathrm{Cu}$ mesh by using a sputtering system to prevent $\mathrm{Ni}$ from being diffused into $\mathrm{Cu}$ mesh substrate during the MWCNT growth process at high temperature, and $\mathrm{Ti}$ reacts with carbon at high temperature, forming $\mathrm{TiC}$ thin layer [34]. But, we speculate that $\mathrm{Ti}$ does not serve as an active material for LIB anode. Also, the average atomic percentages of $\mathrm{C}$ and $\mathrm{Cu}$ elements in four different spots are $99.4 \%$ and $0.4 \%$, respectively. Therefore, most 3D freestanding MWCNTs comprised carbon and their electrochemical performance was strongly dependent on the disordered graphitic structure. Similar to the other lower temperature CVD grown nanocarbon structures, as-prepared MWCNTs contained many defects which could be found from the higher peak ratio of D-band and G-band peaks $I_{D} / I_{G}$ (around 1), as illustrated in Figure 39(e). Figure 39(f) exhibited the SEM image of a MWCNT stack composed of the 8 number of the 3D free-standing MWCNT mesh layers and a single layer of 3D MWCNT on $\mathrm{Cu}$ mesh as a bottom side (The areal density is $34.9 \mathrm{mg} / \mathrm{cm}^{2}$, and the overall thickness is $780 \mu \mathrm{m}$.). In addition, the photographs of the MWCNT anode stacks for the Li-ion coin-cell fabrication are shown in Figure 39(g) which indicates the bottom side of the MWCNT stacked sample was bound with $3 \mathrm{D} \mathrm{Cu}$ mesh current collector in order to enhance the electrical contact between the stacked MWCNTs and the stainless-steel bottom cap of the button cell. Furthermore, to reinforce the bonding strength among MWCNT network in the anode stack, we prepared a binder solution composed of PVDF binder, carbon nanoparticles as conducting agent, and N Methyl 2- 

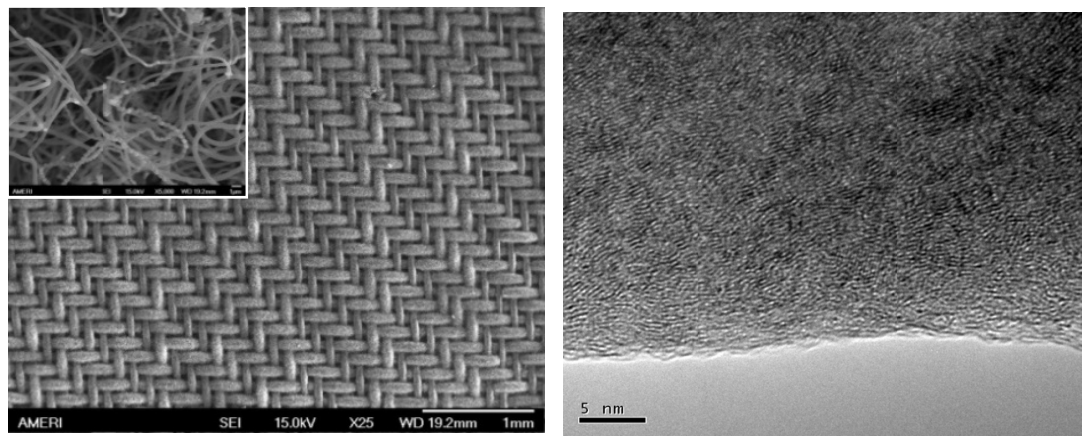

(b)

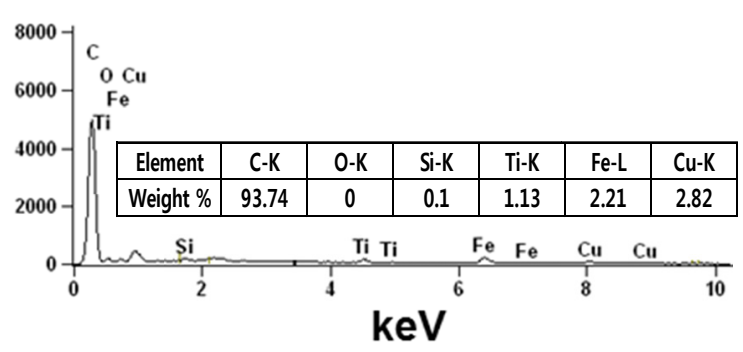

(d)

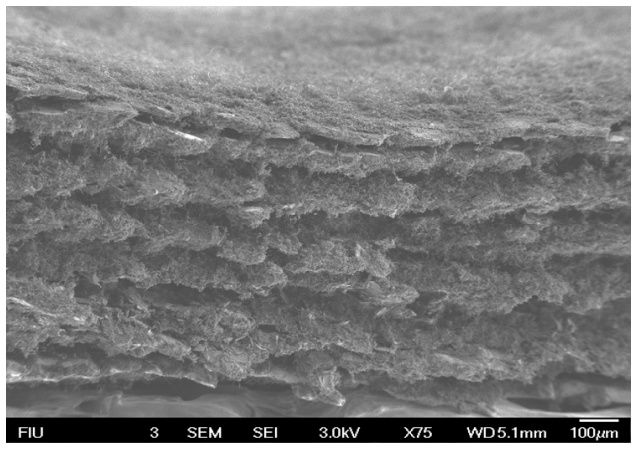

(f)

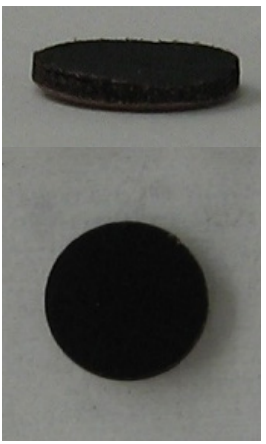

(g)

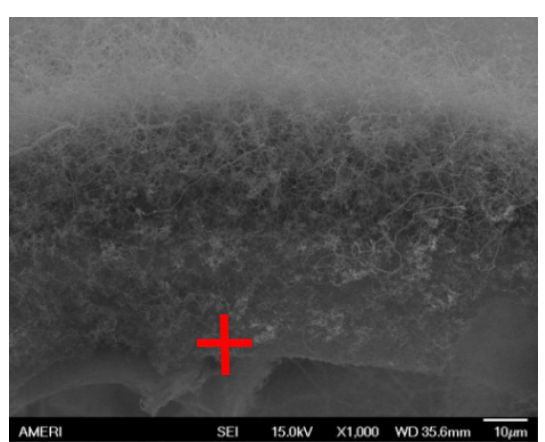

(c)

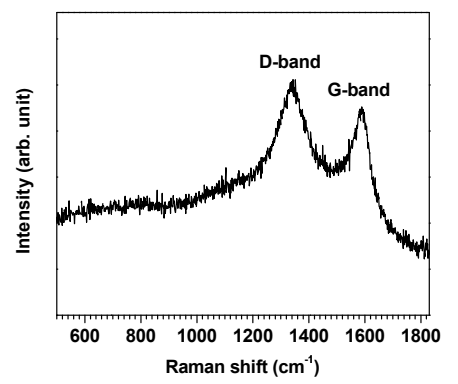

(e)

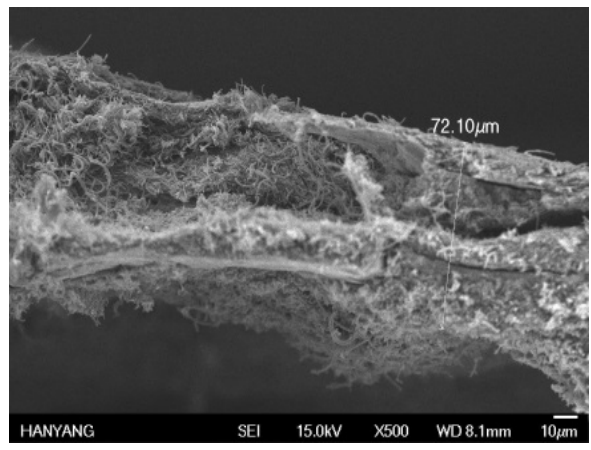

(h)

Figure 39. Structural properties of anode stack composed of 3D MWCNTs presented by diverse analysis techniques. Part (a). SEM plane view of 3D free-standing MWCNT structure. Inset shows higher magnification SEM image of the thread-like MWCNT structure; (b) high resolution TEM (HRTEM) image of a single MWCNT; (c) SEM image indicating the point, denoted by the red cross, where $\mathrm{Cu}$ mesh originally existed before $\mathrm{Cu}$ etching process; (d) EDS spectra of 3D free-standing MWCNTs obtained from the spot indicated by the red cross shown in the SEM image of (c); (e) Raman spectra of 3D MWCNTs; (f) SEM image of the cross-sectioned 
MWCNT-based anode stack showing high areal mass density of MWCNTs around $34.9 \mathrm{mg} / \mathrm{cm}^{2}$; (g) photographs of the final product of anode stack for its button cell assembly; (h) crosssectional SEM image of the binder-assisted and hot-pressed anode stack.

pyrrolidone (NMP) solvent, as mentioned in the experimental section, and applied the binder solution to the anode stack. By compressing the binder assisted anode stack at high temperature and pressure, the 3D MWCNT network structure was strongly bonded, as shown in Figure 39(h). (The areal density is $9.0 \mathrm{mg} / \mathrm{cm}^{2}$, and the overall thickness is $72 \mu \mathrm{m}$ ). PVDF binder was possibly formed as the physical cross-linked structure in the uniform film which holds 3D MWCNT based anode stack network, leading to the maintenance of the electrical conduction throughout the anode stack during cycling [91].

\subsubsection{Electrochemical Properties of MWCNT-Based Anode Stack}

Charge and discharge profiles are very important to understand the electrochemical characteristics of LIB with an anode. As mentioned earlier, a coin-type of cell composed of MWCNT stacked anode and Li metal as counter and reference electrodes is subjected to the charge and discharge cycles at $0.05 \mathrm{C}\left(19 \mathrm{mAg}^{-1}\right)$. As seen in Figure 40(a), the values of the gravimetric specific discharge capacity after lithiation of the anode stack labeled 1 stack (2.6 $\left.\mathrm{mg} / \mathrm{cm}^{2}\right), 5$ stack $(\mathrm{H})\left(9.0 \mathrm{mg} / \mathrm{cm}^{2}\right)$, and 7 stack $\left(18.7 \mathrm{mg} / \mathrm{cm}^{2}\right)$ are $813 \mathrm{mAhg}^{-1}, 905 \mathrm{mAhg}^{-1}$, and $679 \mathrm{mAhg}^{-1}$, respectively. In addition, the values of the volumetric specific discharge capacity after lithiation of the anode stack labeled 1 stack, 5 stack $(\mathrm{H})$, and 7 stack are $231 \mathrm{mAh} / \mathrm{cm}^{3}$, $1,127 \mathrm{mAh} / \mathrm{cm}^{3}$, and $316 \mathrm{mAh} / \mathrm{cm}^{3}$, respectively. We would like to highlight that the value of the gravimetric specific discharge capacity of 5 stack $(\mathrm{H})$ is $111 \%$ and $133 \%$ higher than that of 1 
stack and 7 stack, respectively. In addition, the volumetric specific discharge capacity of 5 stack (H) is $488 \%$ and $357 \%$ higher than that of 1 stack and 7 stack, respectively. Such excellent results could stem from two main reasons. The first reason involves the better electrical contact among MWCNTs through a polyvinylidene fluoride (PVDF) binder and hot pressing. Furthermore, carbon nanoparticles were served as electric conducting agent. The similar principle has been established, and such a binder solution has been commonly used for casting of slurry active materials for LIB [92]. In this study, we would like to emphasize the first application of the principle to the anode stack composed of the 3D free-standing MWCNTs. The second reason involved the morphological variation, such as open-end structures, fractures, and shortened lengths of the MWCNTs after their hot-pressing, and the modified structure could make it more efficient to insert and extract lithium ions into and from the internal structure of MWCNTs, as reported in the previous publication [83, 93]. In Figure 41, the SEM images of the novel hotpressed anode stack qualitatively showed the strong evidences to prove the two reasons. In addition, it should be noted that current anode stack samples were fabricated by relatively lower temperature $\left(750{ }^{\circ} \mathrm{C}\right)$ for MWCNT growth on $\mathrm{Cu}$ mesh using a catalytic thermal CVD method. Since the growth temperature was not high enough to create well-ordered graphitized structure in MWCNTs, MWCNTs contained defective structures composed of carbons present at the layer edges or in smaller polyaromatic domains. The high $\mathrm{I}_{\mathrm{D}} / \mathrm{I}_{\mathrm{G}}$ intensity ratio (around 1.0) as a metric for the degree of graphitization was calculated based on Figure 39(e). The electrochemical behavior is quite different when a mixed structure of disordered and graphitic carbon has not been further heat treated for high degree of graphitization. Such structure contains wide range of possible sites with different energies in which the lithium can reside. The result is that the open circuit potential vs. $\mathrm{Li}^{+} / \mathrm{Li}$ gradually varies, as shown in Figure 40(a), rather than showing the steps characteristic of more ordered structures [94]. Furthermore, it is found that there is considerable irreversible capacity $\left(\mathrm{C}_{\mathrm{irr}}\right)$ loss defined by the difference of specific capacities 


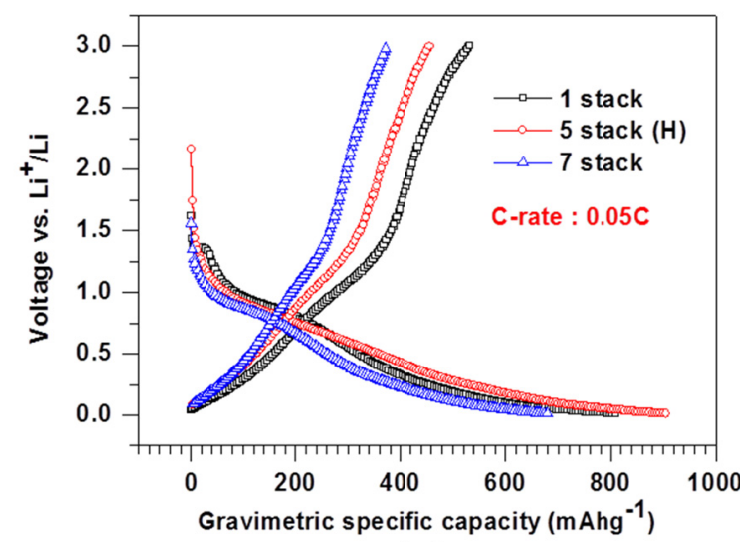

(a)

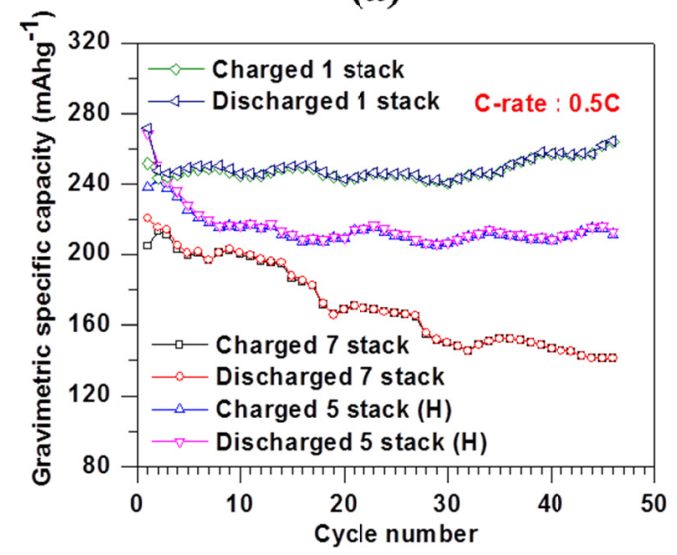

(c)

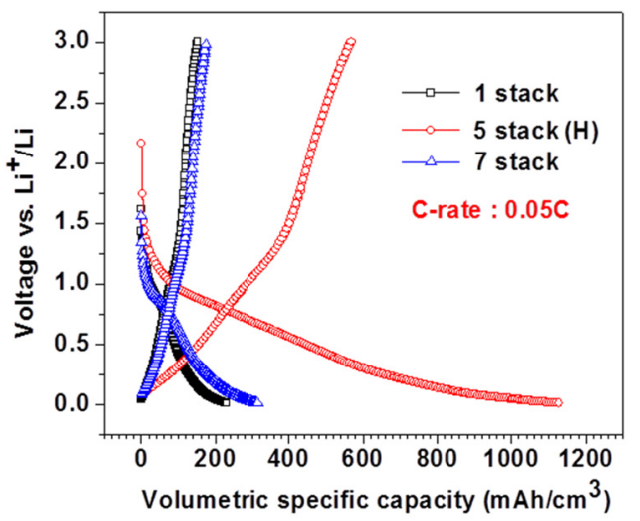

(b)

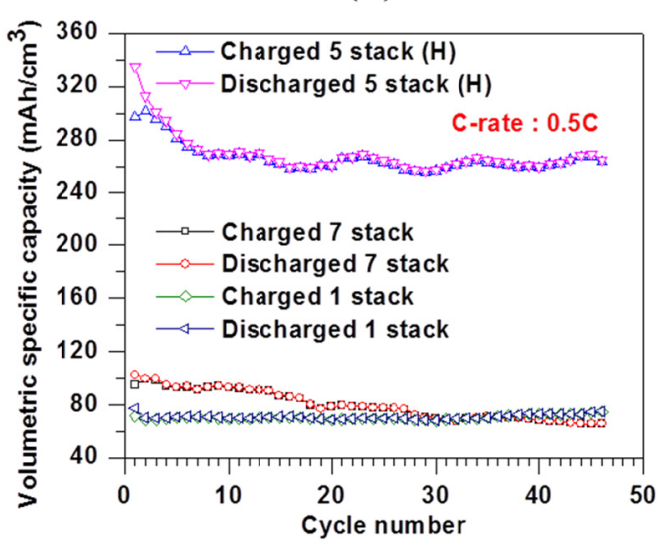

(d)

Figure 40. Electrochemical performance of the anode stacks with different areal loading density of 3D MWCNTs as active materials for LIB application. A primary charge and discharge profile of the anode stacks in terms of (a), gravimetric and (b), volumetric specific capacities at $0.05 \mathrm{C}$. Cycling stabilities of the two different anode stacks with respect to (c), gravimetric and (d), volumetric specific capacities at $0.5 \mathrm{C}$. 


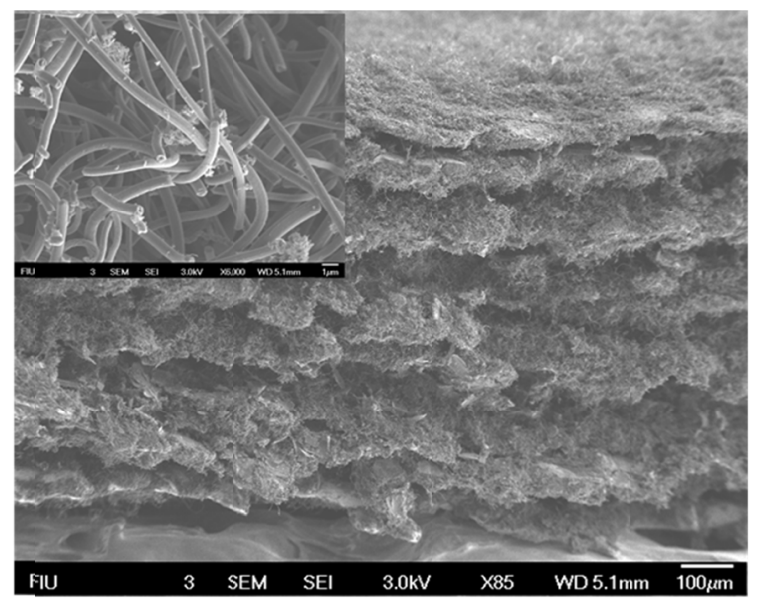

(a)

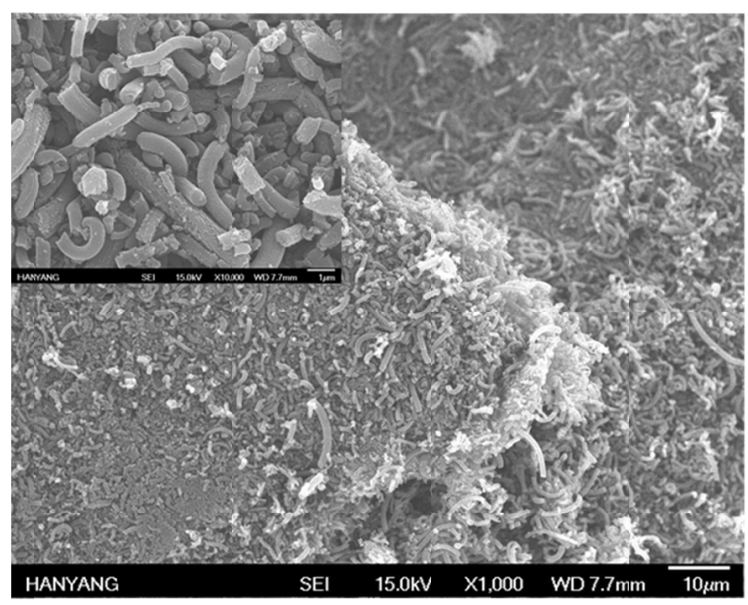

(c)

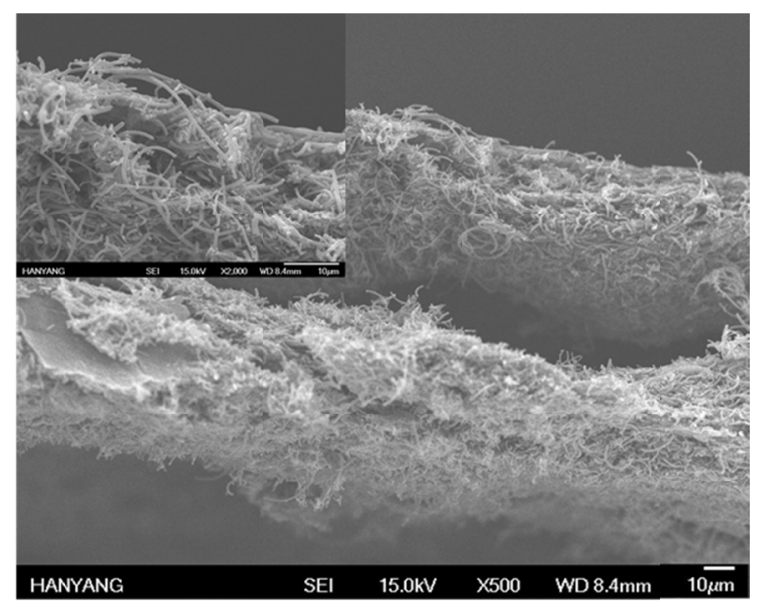

(b)

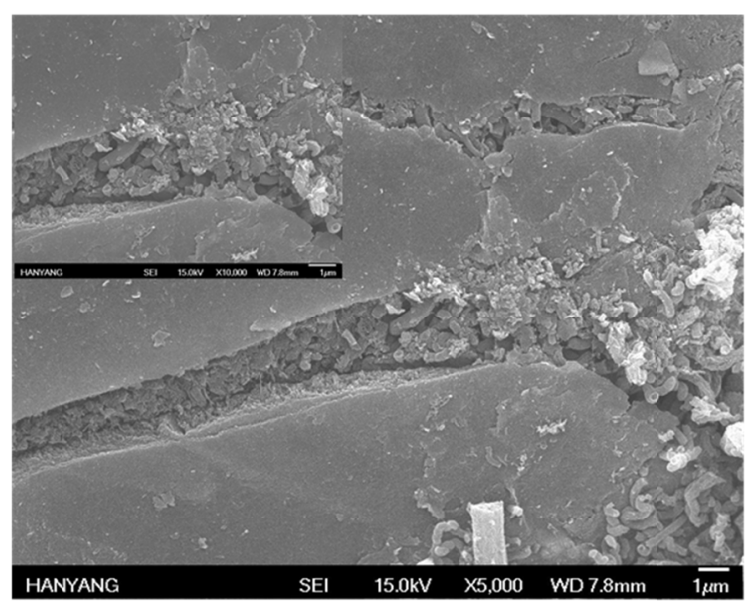

(d)

Figure 41. Cross-sectional FESEM images of (a), mechanically compressed anode stack with the lower pressure and (b), thermal compressed anode stack with the higher pressure; (c-d), planeview FESEM micrographs taken from different regions of the thermal compressed anode stack with higher pressure. The insets show the magnified regions of the FESEM images to identify the morphology of MWCNT network structure. 
obtained during the initial charge and discharge process. The primary reason for the disappointing result is related to the electrolyte decomposition occurred at around $0.8 \mathrm{~V}$, resulting in a distinguishable plateau region, as shown in the initial discharge curve of Figure 40(a) and (b) [95]. This phenomenon is typically observed in the CNT-based anodes [54]. Another reason for the irreversible capacity loss is probably due to trapped $\mathrm{Li}^{+}$ions inside of the anode structure. During charge process followed by discharge, the $\mathrm{Li}^{+}$ions were not completely extracted from the structure and remained.

The cyclability is the essential LIB performance to determine the cycle life of anode and evaluated by number of cycles. Figure 40(c) showed the variation of reversible gravimetric specific capacity $\left(\mathrm{C}_{\mathrm{rev}}\right)$ of the each sample with the different areal mass density as a function of cycle number. The samples of 1 stack and 5 stack $(H)$ presented almost constant capacities. In the case of the anode labeled 1 stack, at $0.5 \mathrm{C}\left(186 \mathrm{mAg}^{-1}\right)$, the gravimetric capacity response with number of cycles showed the increasing wave like trend till the 50th cycle which indicated the creation of free sites for lithium ion migration into the dense entangled CNT network structure due to the electrochemical alteration upon cycling. The sample of 1 stack retained 105\% (264 $\left.\mathrm{mAhg}^{-1}\right)$ of its initial $\mathrm{C}_{\text {rev }} 252 \mathrm{mAhg}^{-1}$, whereas the sample of 7 stack retained $69 \%\left(141 \mathrm{mAhg}^{-1}\right)$ of its initial $\mathrm{C}_{\text {rev }} 205 \mathrm{mAhg}^{-1}$ after the 46th cycles at $0.5 \mathrm{C}$. For the anode labeled 7 stack, the values of the $\mathrm{C}_{\text {rev }}$ kept decreasing as cycling test proceeded. In contrast, the binder-assisted and hot-pressed sample denoted by 5 stack $(\mathrm{H})$ remarkably retained $89 \%\left(211 \mathrm{mAhg}^{-1}\right)$ of its initial $\mathrm{C}_{\text {rev }} 238 \mathrm{mAhg}^{-1}$ after the 46th cycle at $0.5 \mathrm{C}$. The gravimetric and volumetric specific reversible capacities of the sample of 5 stack $(\mathrm{H})$ were $211 \mathrm{mAhg}^{-1}$ and $263 \mathrm{mAh} / \mathrm{cm}^{3}$, respectively, which were comparable to those obtained from the current commercially available $\mathrm{Li}_{\mathrm{x}} \mathrm{C}_{6}$ anodes [96]. According to the results from the samples of 5 stack $(\mathrm{H})$ and 7 stack, we could find the correlation of the strong bonding among MWCNT networks and the cycling stability of MWCNT-based anode stack. The possible reason for the lower values of gravimetric specific $\mathrm{C}_{\text {rev }}$ 
of 7 stack designated sample is associated with the overpotential (IR drop) with the thickness of the anode stack. The thick anode physically connected by the multi-layered free-standing MWCNT meshes intrinsically contained the non-homogeneous voltage distribution in the inner structure of the anode, which resulted in a rate-limit process by $\mathrm{Li}^{+}$ion diffusion in the electrode. That meant different degrees of connectivity of MWCNTs were exhibited so that the distribution of electrical resistances was not uniform throughout the thickness of the anode stack. Furthermore, after the prolonged charge-discharge cycling processes, bonding among the MWCNT network could be loosened. As a result, ohmic resistance in the anode increases, which deteriorates the cycle life of anode. To overcome such a drawback, the sample denoted by 5 stack $(\mathrm{H})$ was composed of well-bonded multi-layers of the free-standing MWCNT meshes by using a two-step process of PVDF based binder application and thermal-compression. The novel hot-pressed anode stack showed the high volumetric density $1.25 \mathrm{~g} / \mathrm{cm}^{3}$, which was comparable to that of current commercially available LIBs [96]. Therefore, the approach of chemical binding and physically strong adhesion effectively reduced the interfacial resistance between neighbor MWCNTs, which was the limitation of anode stack pressed with the lower pressure, and provided charge transfers with multiple conducting pathways [44]. For all the sample cases, the Coulombic efficiency from the charge and discharge capacities was almost $100 \%$ for each cycle number except the first cycle.

\subsection{Conclusion}

We proposed a model to enhance the areal mass density of carbon nanostructures (In our case, multiwall carbon nanotubes (MWCNTs)) for the anode system in high-efficiency LIB. The way to increase the loading amount of MWCNTs was stacking of the several free-standing 3D MWCNT mesh layers after the Cu etching process. Based on the LIB performance tests with the 
fabricated anode stacks, it was found that the lower values of gravimetric specific $\mathrm{C}_{\mathrm{rev}}$ were shown in the sample of higher areal mass density, which was caused by the overpotential (IRdrop and activation and concentration polarization) occurred in the anode stacks. To overcome such limitation, we made a novel binder-assisted and hot-pressed anode stack and confirmed that it showed the excellent LIB performance, which was comparable to that of the commercial available anode. 


\section{CHAPTER 6.}

\section{LARGE SCALE PATTERNABLE 3D CARBON NANOTUBE-GRAPHENE STRUCTURE FOR FLEXIBLE LI-ION BATTERY}

\subsection{Introduction}

Flexible electronics have been considered as next generation portable devices, such as roll/up displays, wearable devices, active radio frequency identification (RFID) tags, integrated circuit smart cards and implantable medical devices. It is expected that flexible Li-ion batteries (LIB) can be used as the energy storage devices for the flexible electronics [44-45]. Currently, several worldwide research teams have prepared nanomaterial-based LIB with the flexible polymer substrates and yielded the excellent LIB performance. V. L. Pushparaj et al. synthesized the flexible nanocomposite thin-film battery comprising CNT-cellulose paper electrode and demonstrated a reversible capacity of $110 \mathrm{mAhg}^{-1}$ in several tens of cycles [46]. J. Chen et al. prepared CVD grown CNT-carbon layer (CL) hybrid anodes for a paper LIB and demonstrated a high reversible capacity of $572 \mathrm{mAhg}^{-1}$ after 100 cycles [47]. M. Kaempgen et al. fabricated printable thin film supercapacitor by using single-walled carbon nanotubes (SWCNTs) spray deposited onto PET films and attained high specific capacitances in the range from $\sim 90$ to 120 F/g by using various aqueous electrolytes [48]. However, these previous results have some drawbacks, such as lower film adhesion properties of the active materials without compromising their electrochemical performance and employment of complicated coating process. Nevertheless, higher specific and volumetric capacities of the anodes have been motivated for the development of emerging flexible electrodes without metallic current collectors or even polymer substrates (e.g. SWCNT papers) [43]. 
Another major research into LIB has been currently focused on high-efficiency LIB composed of nanomaterial-based electrodes for the next generation electric vehicles (EVs) as one of the most attractive application. The strong candidates of the nanomaterials can be listed as carbon nanotubes (CNTs) [10], carbon nanofibers (CNFs) [97-98], graphene [11], Si nanowires [12], transition metal oxide nanoparticles [13] and so on. In particular, the LIB cell assembled by those nanostructured anodes have demonstrated their excellent electrochemical properties with respect to safety, high energy density, C-rate capability and long cycle lifetime relative to those of the conventional graphite anodes. In our previous study, we used directly grown multiwall carbon nanotubes (MWCNTs) on metal films with major advantages over conventional mesocarbon microbeads (MCMB) or hard carbon-based electrodes since MWCNTs have their unique properties, including high surface area to accommodate more $\mathrm{Li}^{+}$ion intake, good electric conductivity for the faster electron charge transfer and good structural resilience for $\mathrm{Li}^{+}$ion insertion and extraction into and from the host structure $[26,99]$. However, it is difficult to obtain high volume or mass loading density of nanostructured active materials for their real application by using their conventional coating or direct growth method on the $2 \mathrm{D}$ metal substrate [36]. To overcome the limitation, there have been many reports to show $3 \mathrm{D}$ electrode architectures to be applied to supercapacitor and LIB. H. Zhang et al. prepared 3D network electrode structure for effective electron and ion pathways and assembled a prototype lithium-ion pouch battery, comprised of a bicontinuous lithiated $\mathrm{MnO}_{2}$ cathode and a graphite anode. They demonstrated relatively high specific capacity value (c.a. $110 \mathrm{mAhg}^{-1}$ ) obtained from charge and discharge cycling at 60C [38]. Very recently, direct growth of CNT-graphene seamlessly grown on 3D Ni foam was reported, demonstrating the field emitter and supercapacitor applications of the novel structure [100]. In the structure, the use of 3D Ni foam can promote the surface-area-utilization efficiency of the metal current collectors and graphene can serve as a linking agent between CNTs and metal interface to improve the bonding strength and electrical properties between the 
two phases. Additionally, MWCNTs directly grown on 3D Cu mesh anode architecture has been proposed by Dr. Wonbong Choi's group at FIU and the anode demonstrated not only mass loading density of MWCNT as active materials is four times higher than that of MWCNTs grown on $2 \mathrm{D} \mathrm{Cu}$ but also $160 \%$ improvement of specific capacity in the cycling performance with 254 $\mathrm{mAhg}^{-1}$ at 50th cycle [81].

In this chapter, we propose the architecture of large-scale patternable 3D MWCNTs-graphene hybrids transferred over transparent flexible films, where graphene functions as an underlayer film to enhance the electrical conductivity for 3D MWCNT network structure. The 3D MWCNT structure was transferred by a roll-to-roll lamination process and formed as a patterned structure of 3D carbon nanotubes (CNTs)-graphene [99]. As-prepared 3D MWCNTs-graphene-PET structure was subjected to electrochemical performance tests with a coin-cell type of LIB. In addition, it is proved that the novel structure can be available for high efficiency flexible LIB. It is noted that the fabricated 3D MWCNTs-graphene-PET anode does not include any binders which may have harmful effect during the LIB operation. Furthermore, flexing of 3D MWCNTsgraphene on PET substrates leads to no significant film cracking or peel-off, which is inconsistent with the previous report [43]. Moreover, a commonly used roll-to-roll lamination method implies the potential for high-throughput production of the novel 3D MWCNTs-graphene-PET anode structure.

\subsection{Results and Discussion}

\subsubsection{Experimental Details}

A Cu mesh (200 Mesh Copper, TWP) with average dimensions of $50 \mu \mathrm{m}$ thickness and wire size and $65 \mu \mathrm{m}$ hole size was prepared. 3D multiwall carbon nanotubes (MWCNTs) grown on $\mathrm{Cu}$ 
mesh was used as anodes for Li-ion battery. The anode materials were prepared by a two-step process, including $\mathrm{Ti}$ (underlayer, $\sim 10 \mathrm{~nm}$ thickness)/Ni (catalyst, $2.5-10 \mathrm{~nm}$ thickness) thin film deposition on the $\mathrm{Cu}$ mesh by using a radio frequency (RF)-direct current (DC) magnetron sputtering system followed by direct growth of MWCNTs through thermal chemical vapor deposition (CVD). In the first step, Ti underlayer played an important role in preventing Ni thin layer from being diffused into the $\mathrm{Cu}$ mesh underneath, maintaining activity of catalytic $\mathrm{Ni}$ thin layer. In addition, formation of titanium carbide (TiC) thin layer at around $750{ }^{\circ} \mathrm{C}$ could improve electrical conductivity and be an effective bonding bridge between MWCNTs and $\mathrm{Cu}$ mesh. The presence of a TiC thin layer was evidenced in the interface between MWCNTs and $\mathrm{Cu}$, which was previously confirmed by HRTEM $[34,66]$. In the second step, the Ti/Ni deposited $\mathrm{Cu}$ mesh was rapidly heated to $750{ }^{\circ} \mathrm{C}$ in an Ar gas environment with very low humidity level. Carbon precursor ethylene $\left(\mathrm{C}_{2} \mathrm{H}_{4}\right)$ gas carried by hydrogen $\left(\mathrm{H}_{2}\right)$ gas $\left(1: 2\right.$ volume ratio of $\mathrm{C}_{2} \mathrm{H}_{4}$ to $\left.\mathrm{H}_{2}\right)$ was fed into the chamber for growth time 50 minutes. Once the growth time was reached, mixture gases of ethylene and hydrogen were turned off and the chamber was cooled down to the room temperature by flowing inert Ar gas.

We have introduced a simple hot lamination process to transfer MWCNTs grown on 3D $\mathrm{Cu}$ mesh onto graphene laminated polyethylene terephthalate (PET) flexible substrate. Graphene film was synthesized by a CVD method [101]. A commercially available $\mathrm{Cu}$ foil of 50 to $200 \mu \mathrm{m}$ thickness (Nimrod Hall Copper, 99.9\% purity) was first annealed at $1,000{ }^{\circ} \mathrm{C}$ for 1 hour under an Ar gas atmosphere. After annealing, $\mathrm{Cu}$ foil was acid-treated for 10 minutes in $1 \mathrm{M}$ acetic acid at $60{ }^{\circ} \mathrm{C}$ to remove oxide layer created on the $\mathrm{Cu}$ foil surface during the annealing process. Subsequently, copper foils were thoroughly washed with de-ionized water and dried at the ambient conditions. Graphene on the $\mathrm{Cu}$ foil was synthesized at $1,000{ }^{\circ} \mathrm{C}$ and 1 atm pressure, using a 5 minute flow of $\mathrm{CH}_{4}$ and $\mathrm{H}_{2}$ gases in $1: 4$ ratios. At the end of this process, the foil was cooled down to room temperature. We used a hot-press lamination and a chemical etching 
process for transferring graphene grown on the $\mathrm{Cu}$ foil to a transparent flexible PET substrate. A $\mathrm{Cu}$ foil with graphene was hot-press rolled with a transparent flexible PET film having thickness $\sim 50 \mu \mathrm{m}$. To completely remove $\mathrm{Cu}$ from the graphene and laminated film, a concentrated $\mathrm{FeCl}_{3}$ solution was used. The laminated PET film with graphene and $\mathrm{Cu}$ foil underneath was floated over the $\mathrm{FeCl}_{3}$ acid bath at room temperature. After 40 minutes of the etching process, $\mathrm{Cu}$ was completely dissolved into the solution, leaving graphene film with the PET substrate. The resultant transparent flexible film was thoroughly washed with de-ionized water and dried in ambient conditions. In addition, Figure 42 shows the processing flow for each step for the transferring of 3D MWCNTs on the graphene-PET films. The as-grown MWCNTs/Cu mesh and the graphene-PET were held in a "face-to-face" orientation. In the similar manner, the hybrid samples of 3D MWCNTs-graphene-PET were hot press rolled at around $70{ }^{\circ} \mathrm{C}$. After the lamination process, MWCNTs were strongly adhered to the graphene on PET films. In order to completely remove the $\mathrm{Cu}$ mesh from the sandwich-like hybrid structure, we used concentrated $\mathrm{FeCl}_{3}$ etching solution (Transene Company, Inc.). Laminated PET films with 3D MWCNTsgraphene and $\mathrm{Cu}$ mesh underneath were floated over $\mathrm{FeCl}_{3}$ acid bath at room temperature. After longer than four hours, the $\mathrm{Cu}$ mesh was completely etched away, leaving 3D MWCNT mesh patterns transferred onto graphene on PET films. Such a patterned hybrid structure was thoroughly washed with de-ionized water and dried in the oven at $80{ }^{\circ} \mathrm{C}$ for 15 minutes. As presented in the inset of Figure 42(e) and Figure 43, the as-prepared structure was flexible enough to form various geometries without any damage to the MWCNT patterns. The primary reason behind this is the important role of pressure-sensitive adhesives coating of the PET films to make it possible to strongly bond between MWCNTs-graphene and PET. 


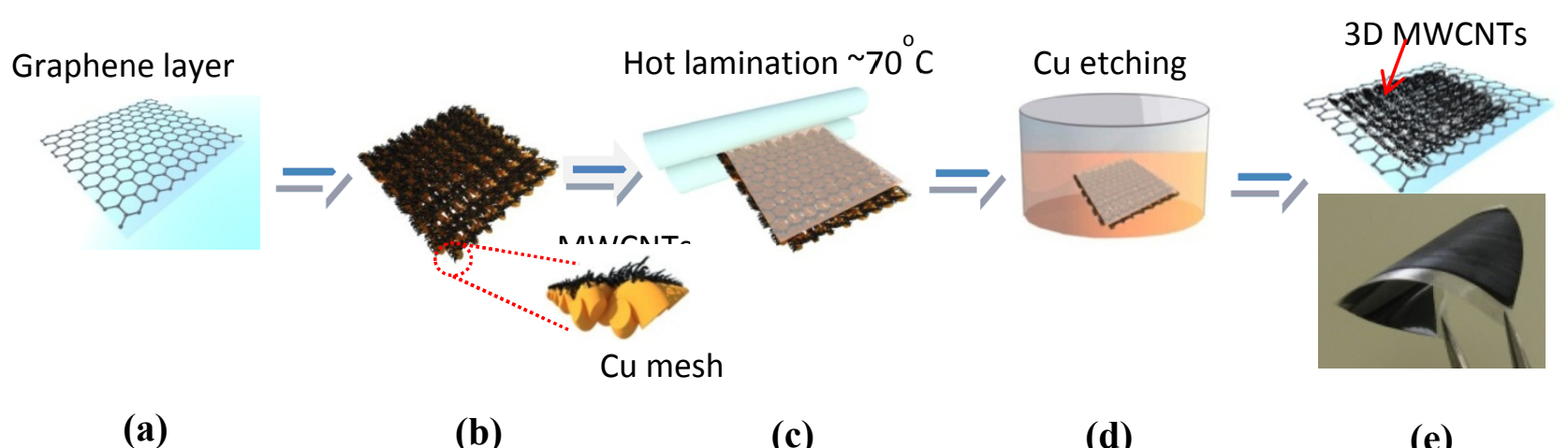

Figure 42. Process flow of transferring techniques of 3D MWCNTs/Cu mesh onto the graphene/ transparent flexible PET substrate. Part (a). Graphene transferred onto PET film, (b) 3D MWCNTs on $\mathrm{Cu}$ mesh directly grown by CVD, (c) hot lamination of 3D MWCNTs/Cu mesh with graphene-PET, (d) etching process of $\mathrm{Cu}$ mesh by using $\mathrm{FeCl}_{3}$ oxidation acid, (e) 3D MWCNTs pattern transferred onto the graphene-PET film. Inset shows a digital image of high flexibility of the 3D MWCNTs transferred onto the graphene-PET films.

Surface morphology and structural properties of the prepared 3D MWCNTs-graphene-PET structures were observed using a field emission scanning electron microscope (FESEM) (JEOL, JSM-7000F; HITACHI, S-4800), an energy dispersive spectroscope (EDS) (Thermo Electron Corporation, NORAN System SIX), a micro Raman system (UniG2D, Korea; see Figure 28) (laser source : low noise $532 \mathrm{~nm}$ Diode-pumped solid-state (DPSS) laser (3.7 mW); software : iDus CCD detector) and a field emission transmission electron microscope (FETEM) (FEI, TECHNAI F20). Electron conductivity of the structure was measured using a four-probe station (MS Tech, Korea) connected to a digital multi-meter (Keithley, 2400). Measurements were performed after flexing the samples at different bending angles, ranging from $0^{\circ}$ to $180^{\circ}$. 

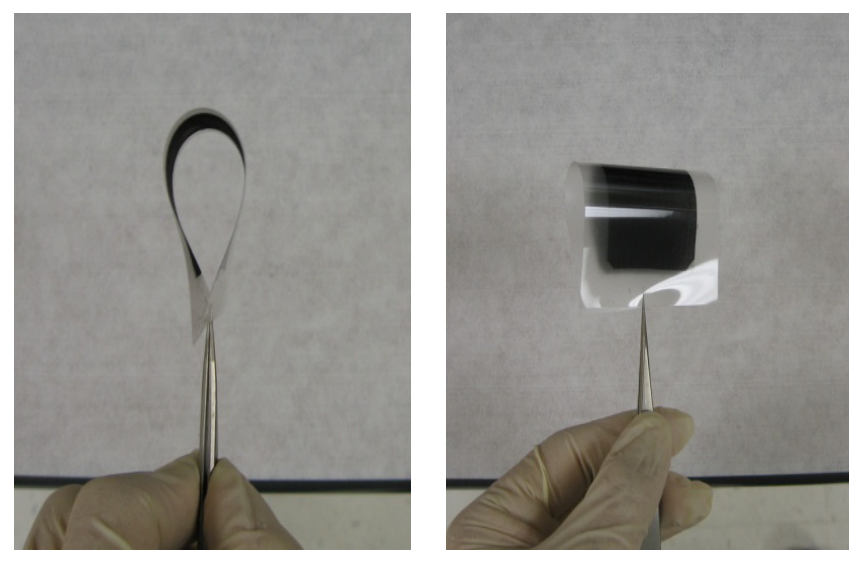

Figure 43. Digital images of the 3D MWCNTs-graphene-PET structure.

Prior to using the as-prepared structure as an anode for Li-ion battery, the anode was cut into a disk with $14 \mathrm{~mm}$ diameter and dried by heating at $50{ }^{\circ} \mathrm{C}$ under high vacuum for longer than 12 hours to ensure complete water removal. For the electrochemical performance tests, a coin type (CR 2032) of half-cell assembly was carried out in an argon filled glovebox under extremely low level of humidity $(<1 \mathrm{ppm})$. The 3D MWCNTs-graphene-PET structure was used as working electrode, whereas Li metal foil as both counter and reference electrodes. PET acted as a flexible substrate for the 3D MWCNTs-graphene, however due to its insulating behavior, a crescent shaped $\mathrm{Cu}$ current collector was used to cover the edges of the electrode in order to make electrons feasibly conduce between the active 3D MWCNT materials and the bottom of coin cell. $1 \mathrm{M}$ solution of $\mathrm{LiPF}_{6}$ salt in 1:1 (v/v) mixture solvent of ethylene carbonate (EC) and dimethylene carbonate (DMC) was used as electrolyte. Typical polypropylene (PP) material (Separator 2400) was used as separator. The cell was finally assembled by using a CR 2032 coin cell crimping tool (Honsen Corp.). The charge and discharge tests of the assembled cells were 
performed in a multi-channel battery testing unit (MACCOR) at room temperature $\left(\sim 25^{\circ} \mathrm{C}\right)$ in galvanostatic mode (constant current density).

\subsubsection{Structural and Electrical Properties of 3D MWCNTs-Graphene-PET}

Recently, it has been proposed that 3D anode structure for LIB has significantly improved mass amount of electrochemical active materials for ion and electron transport [39-40]. MWCNTs-graphene hybrid anode has been attracted a great attention as anode materials for LIB since their unique morphology and structure have advantages over other reported anodes, such as high electrochemical active area, good electric conductivity and structural adjustment for $\mathrm{Li}^{+}$ion insertion and extraction. In Figure 44(a), SEM image showed regularly patterned 3D MWCNT structure was successfully transferred and uniformly distributed over the graphene on PET film. Two-dimensional EDS mapping for the carbon distribution was illustrated in Figure 45(a). Note that the red-color arrow, which represents the length and direction of linear scan of EDS for elemental analysis for the selected area (see Figure 45(b)). From the two spots of 3D MWCNTs on graphene-PET film, EDS spectra represented higher than 99 atom\% of carbon and less than 0.2 atom $\%$ of copper (Figure 45(c)). Zoomed-in SEM plane view of the transferred 3D MWCNTs on PET structure was shown in Figure 44(b). The structure was composed of the lower MWCNT density region in the four square shaped areas which had been originally holes in the pristine $\mathrm{Cu}$ mesh and the higher MWCNT density region were originally grown on the interwoven $\mathrm{Cu}$ wires before the $\mathrm{Cu}$ etching process. As the loading density of MWCNTs increased, the more MWCNTs were filled, creating high density pillars and the transparency of the 3D MWCNTsgraphene-PET film accordingly decreased. In our knowledge, such patterned 3D MWCNTsgraphene-PET films were unique structures compared to the previous result showing MWCNTs transferred over polycarbonate (PC) film by a rolled quartz cylinder [99]. The promising 
advantages of PET as a substrate are its flexibility, good adherence with active materials and ease of transferring of thin layers like graphene without structural degrading for its application to the flexible electronic devices. Some researchers previously used PET as a flexible substrate in order to fabricate supercapacitors $[48,99]$. Also, it should be noted that our proposed anode structure is distinct in several perspectives compared to the previously patterned 3D structure which was prepared by using a small scale laser-jet patterning technique [99]. We employed a commercially available copper mesh, which could be easily etched away and scalable, with roll-to-roll fashion. As presented in Figure 44(c), the high magnification SEM image shows the randomly oriented 3D MWCNT structure grown by thermal CVD and the captured 3D MWCNTs are their diameters in the range of $200 \mathrm{~nm}$ to $300 \mathrm{~nm}$ which are relatively higher than those in the previous results around $100 \mathrm{~nm}[34,71]$. Damage of the PET films by electron beam irradiation limits the observation of the transferred graphene on PET. As illustrated in Figure 44(d), the 3D MWCNTs are strongly bonded to the PET film with their average length around $40 \mu \mathrm{m}$ and such adhesion is due to the patent pressure sensitive adhesion layer coated on the commercial PET film. The schematic of the cross-sectional image and the description for each component of the 3D MWCNTs-graphene-PET anode structure are presented in Figure 44(e).

In contrast, polymer binders or adhesives have been used to enhance the bonding strength between nanomaterial-based film and polymer flexible substrates, but these can also decrease in the electric conductivity of the film [43]. Thus, our binder-free transfer process can result in low cost and high-throughput. It is investigated that the real bonding strength of graphene and CNTs is negligibly weak so that the adhesion layer can physically attach the both non-attractive materials. During a thermal lamination process, the PET film is under the condition of high pressure and temperature around $70{ }^{\circ} \mathrm{C}$ and the adhesion layer functions as a glue between MWCNTs and graphene on PET film. HRTEM image reveals that graphene layer stacking inside 
a MWCNT of $\sim 300 \mathrm{~nm}$ diameter is randomly oriented (turbostratic structure), forming internal layer structure instead of being aligned parallel to each other (co-centric tube structure), as shown

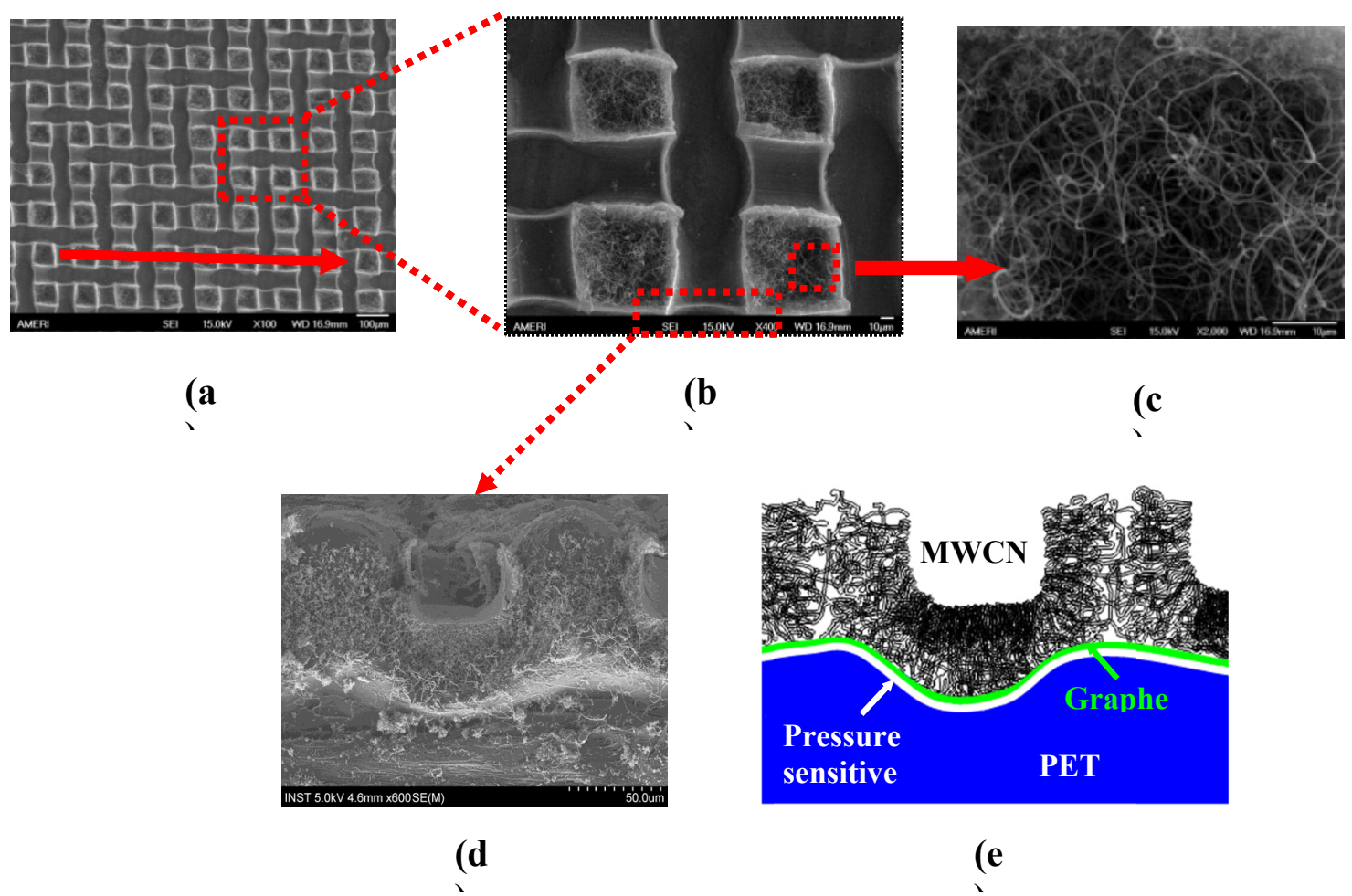

Figure 44. Structural analysis of as-synthesized 3D MWCNTs-graphene-PET structure. (a) The magnified SEM image, (b) Zoomed-in SEM image showing detailed morphology of the structure, (c) The magnified SEM image of the randomly oriented CNF structures in the pattered structure, (d) Zoomed-in SEM cross sectional image, (e) Schematic diagram (not to scale) of the cross sectional image shown in Figure 44(d). 


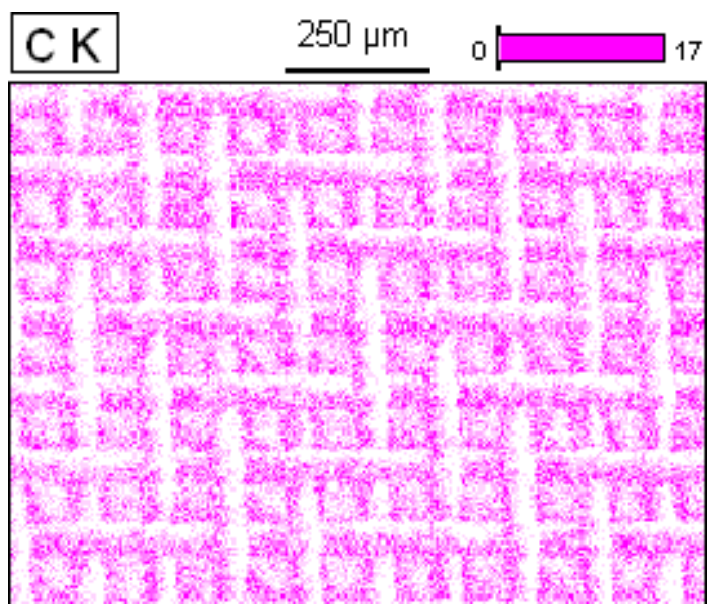

(a)

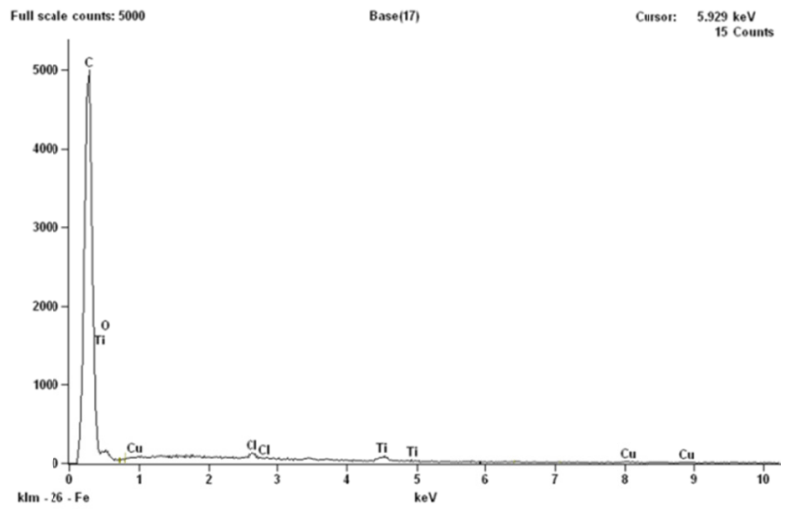

(c)

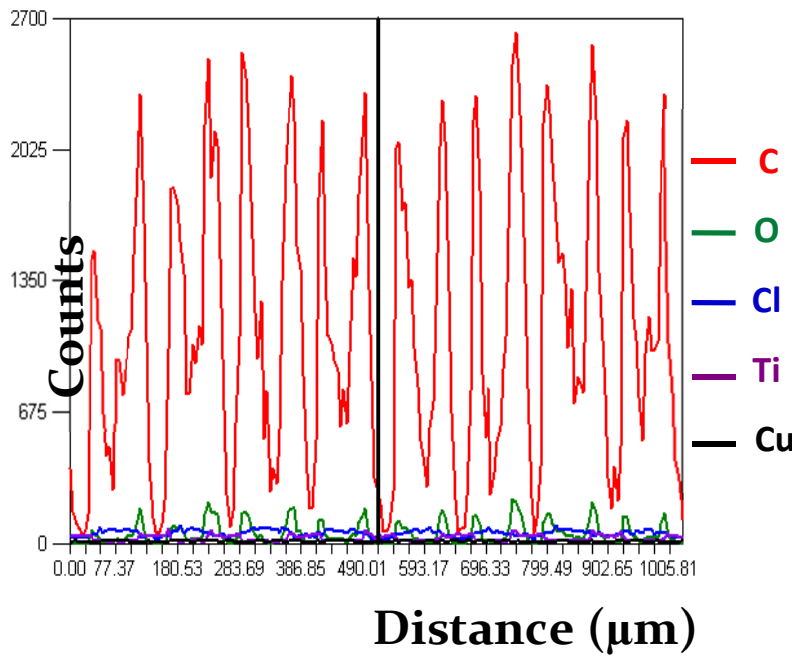

(b)

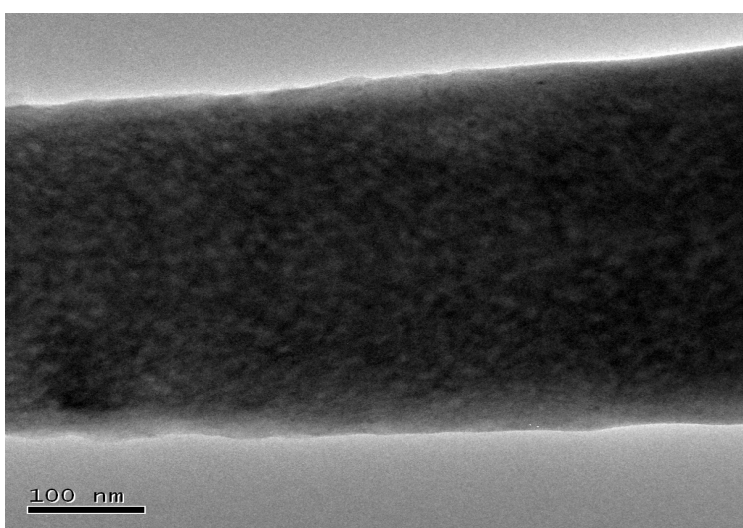

(d)

Figure 45. (a) 2 dimensional EDS mapping for the carbon distribution in Figure 44(a), (b) Line scanned EDS mapping according to the red colored arrow presented in Figure 44(a) showing the presence of the predominant carbon element, (c) EDS spectra of a spot of 3D MWCNTs on graphene-PET film showing higher than 99 atom\% carbon and less than 0.2 atom\% of copper, (d) An HRTEM image of the randomly oriented distribution of the graphene layer in a MWCNT. 
in Figure 45(d). Importantly, it may be recalled here that structural integrity without defilement and retention of the electrical property of electrochemical active materials are essential for their flexible Li-ion battery performance. Considering the insulating PET film substrate, it is very difficult to expect the electron conductivity through the 3D MWCNTs-graphene-PET structure, whereas 3D MWCNT networks in this study shows high electric conductivity (low sheet resistance $\sim 95 \Omega / \mathrm{sq}$ ) which is comparable to $\sim 40-50 \Omega /$ sq obtained from the highly SWCNTspray coated PET substrates [48]. Therefore, 3D MWCNT networks along the structure can be used as both current collectors and electrochemical active materials. Furthermore, true flexible nature of the structure is strongly related to the complete elastic deformation after severe bending. To examine such requirements, the sheet resistance of the 3D MWCNTs-graphene-PET structure was measured as a function of bending angle. As a result, even after severe flexing around $180^{\circ}$, the normalized sheet resistance with respect to that obtained from the structure without bending $\left(\mathrm{R}_{\mathrm{deg}} / \mathrm{R}_{\mathrm{o}}\right)$ is around 1 (see Figure 46$)$. The structural integrity and the retention of electrical properties are presumably associated with the combined effect of the flexibility of individual MWCNTs, the strong adhesion of the MWCNTs to the PET films, and the macroscopic porous morphology of the 3D MWCNT structure, releasing the bending strain. Based on the finding, it is noteworthy mentioning here that our proposed novel 3D MWCNTs-graphene-PET structure meets key requirements for flexible Li-ion battery. In many cases, MWCNTs and graphene are characterized by two main features which are the G mode, coming from the first-order scattering of the $\mathrm{E}_{2 \mathrm{~g}}$ phonon of $\mathrm{sp}^{2} \mathrm{C}$ atoms (usually observed at $\sim 1580 \mathrm{~cm}^{-1}$ ) and the $\mathrm{D}$ mode, a breathing mode of k-point photons of $A_{1 g}$ symmetry $\left(\sim 1350 \mathrm{~cm}^{-1}\right)$ [97]. Raman results also show the typical G and D peaks presented in Figure 47(a) and (b), indicating that graphene and MWCNTs are successfully CVD grown on $\mathrm{Cu}$ and transferred over PET. Figure 47(b) presents the magnified Raman spectra from graphene-PET structure and pristine PET film to clearly identify D and G mode peaks. In addition, it is found that the MWCNTs contain defective structure based 


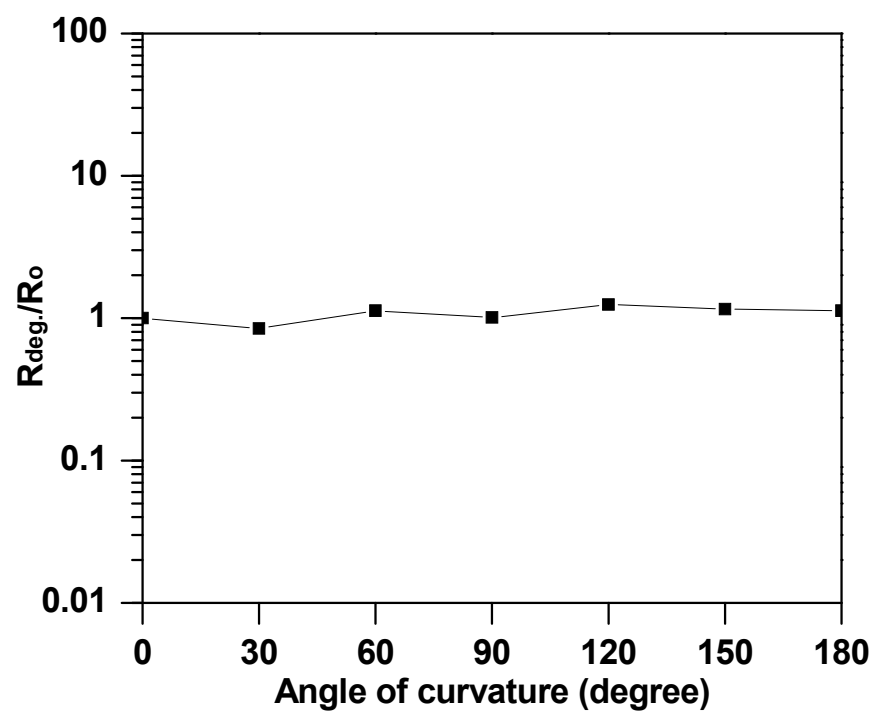

Figure 46. Variation in the normalized resistance $\left(\mathrm{R}_{\mathrm{deg}} / \mathrm{R}_{\mathrm{o}}\right)$ as a function of the angle of curvature.

on the ratio of the intensity of D-band to that of G-band around 1 which is presumably due to the relatively lower temperature $\left(750{ }^{\circ} \mathrm{C}\right) \mathrm{CVD}$ synthesis. Unlikely, the graphene on $\mathrm{Cu}$ and PET shows 2D peak appearing at around 3,000 $\mathrm{cm}^{-1}$ Raman shift, which is different from the typical $2 \mathrm{D}$ mode at $2,700 \mathrm{~cm}^{-1}$. 


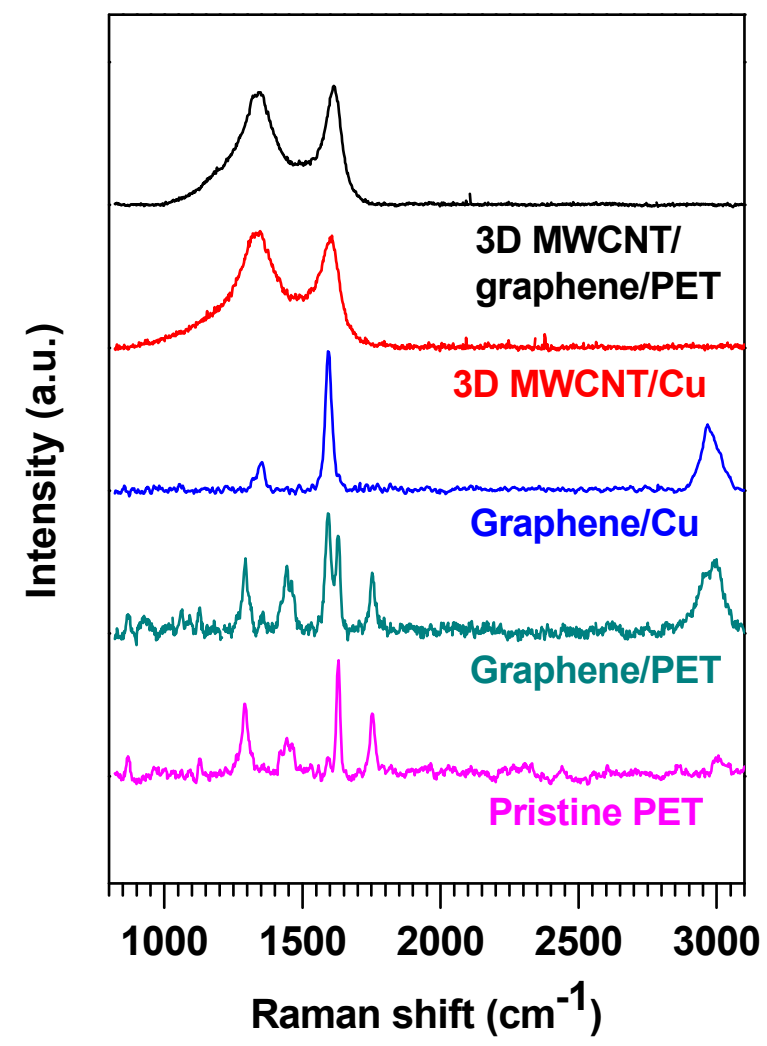

(a)

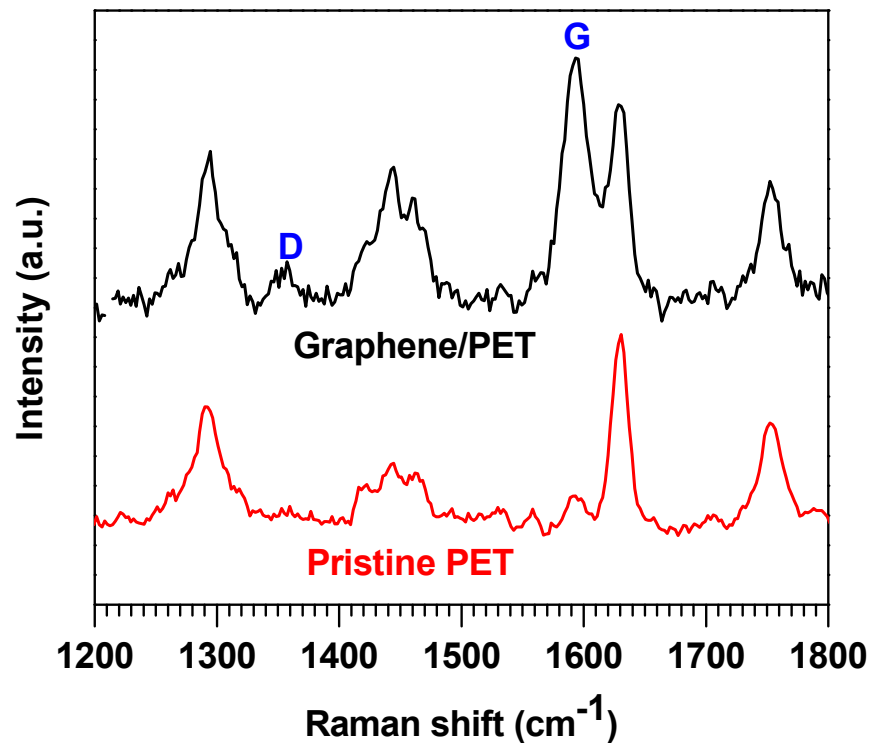

(b)

Figure 47. Raman spectra of (a) 3D MWCNTs and graphene on two different substrates Cu foil and PET film and pristine PET film and (b) magnified Raman spectra of graphene on PET and pristine PET film. 


\subsubsection{Li-ion Battery Performance of 3D MWCNTs-Graphene-PET}

Electrochemical performance using a half-cell, which is composed of the 3D MWCNTsgraphene-PET structure as working electrode and Li metal foil as reference and counter electrode is shown in Figure 48. Initial charge and discharge cycle, measured at a current rate of $63 \mathrm{mAg}^{-1}$ $(0.17 \mathrm{C})$, is shown in Figure 48(a). Primary lithiation and delithiation capacities for the 3D MWCNTs-graphene-PET sample are $293 \mathrm{mAhg}^{-1}\left(\mathrm{LiC}_{7.6}\right)$ and $153 \mathrm{mAhg}^{-1}\left(\mathrm{LiC}_{14.6}\right)$, respectively and the corresponding Coulombic efficiency (irreversible capacity) is around 52\%. The values of the discharge and charge specific capacities are lower than those obtained from 3D MWCNTs on $\mathrm{Cu}$ mesh anode systems [81], but they are still comparable with a reversible capacity of 110 $\mathrm{mAhg}^{-1}$ from CNT-cellulose paper electrode [46]. Even though PET is a good flexible substrate for 3D MWCNTs-graphene, the electronically insulating behavior of the substrate acts as a barrier for the effective electronic transport. As mentioned in the experimental section, instead of using crescent shaped electrode on the top edges of MWCNTs to enforce redox reactions, it would be recommended to insert the electrode during the graphene-PET lamination process in order to obtain improved electrochemical performance. However, due to the lack of facilities to fabricate flexible batteries which normally require sophisticated tool, in this circumstance, we tested by using coin cells to show the performance of the electrode as demonstrated in the experimental section.

In addition, the low sheet resistance $(95 \Omega / \mathrm{sq})$ of the anode plays a crucial role to activate the electrochemical redox reactions between current collector and MWCNTs-Graphene. The primary reason for the relatively higher irreversibility is related to the electrolyte decomposition occurred around $0.8 \mathrm{~V}$ resulting in a plateau region as shown in the initial lithiation curve of Figure $48(\mathrm{a})$ and the larger surface area of the 3D MWCNTs. This phenomenon is typically observed in the carbon nanostructure-based anodes in the previous reports $[14,86]$. Graphene is known as high 
potential anode materials for LIB with excellent LIB performance in the previous reports [102]. It should be highlighted that the graphene layer lying between 3D MWCNTs and PET film is too light to be considered practical electrochemical active material for $\mathrm{Li}^{+}$ion insertion and extraction into and from the graphene structure.

Figure 48(b) presents the cycling stability for the 3D MWCNTs-graphene-PET anode structures in long-cycle operation as one of the key electrochemical properties for LIB. Specific capacity of the anode has been recorded for 50 cycles at $1.7 \mathrm{C}$ (current density of $632 \mathrm{mAg}^{-1}$ ). The lithiation capacity slightly increases from $96 \mathrm{mAhg}^{-1}$ at the 1 st cycle to $130 \mathrm{mAhg}^{-1}$ at the $47 \mathrm{th}$ cycle and then gradually decreases to $124 \mathrm{mAhg}^{-1}$ until the 50th cycle. Such a variation of the capacity over the whole cycles is inconsistent with the constant capacities as a function of cycle number shown in our past results [34-35]. It is speculated that one possible reason for such increasing trend of capacity upon number of cycles is associated with the more electrochemical active area on the densely entangled 3D MWCNTs structure created by the continuous charge and discharge process. In our previous publication, it was found that no severe defect or structural damage appeared in the 150 cycled 3D MWCNT-based anode system through observing FESEM and HRTEM images [81]. In the whole charge and discharge cycles, Coulombic efficiency is higher than $98 \%$, indicating the high reversibility of our proposed anode system (see Figure 48(c)). The high reversibility of feasible $\mathrm{Li} / \mathrm{Li}^{+}$redox reactions might be due to the $3 \mathrm{D}$ structure of MWCNTs and co-centric graphene covered MWCNT's formation at the bottom of the MWCNT which paves ease of lithium ion insertion and extraction. The 3D structure is able to accommodate more lithium ion in the MWCNT structure since electrolyte penetrates into the structure and spread over everywhere so that lithium ion can be facilely transported back and forth. Also, 3D structure could avoid SEI inactive layer formation due to the porous structure to allow electrolyte to reach the outer walls of MWCNTs to the core. 


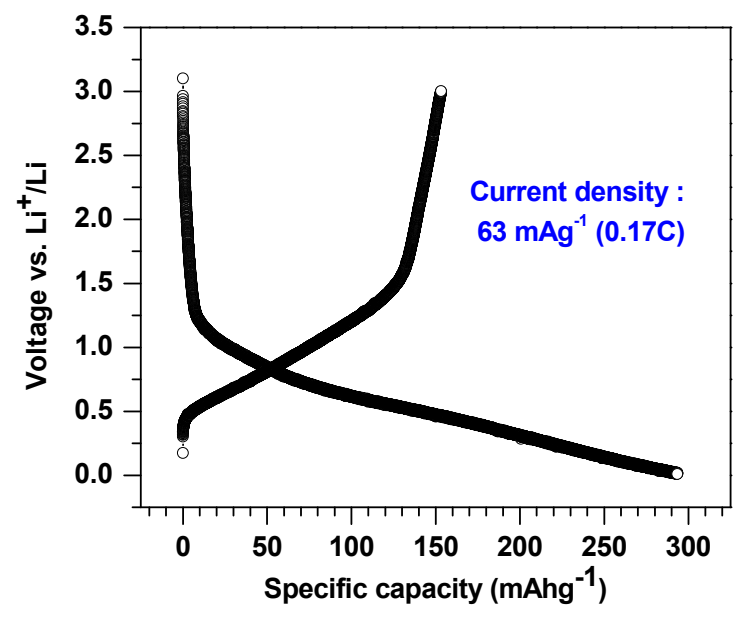

(a)

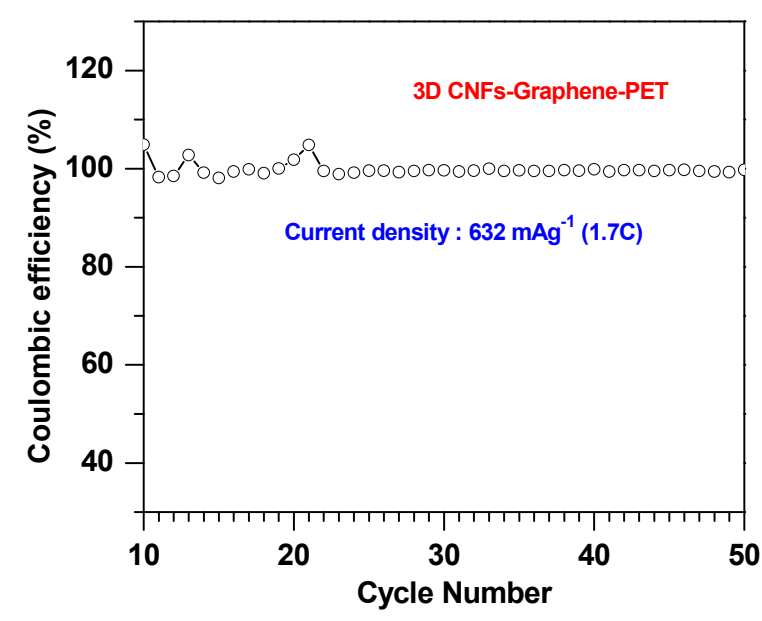

(c)

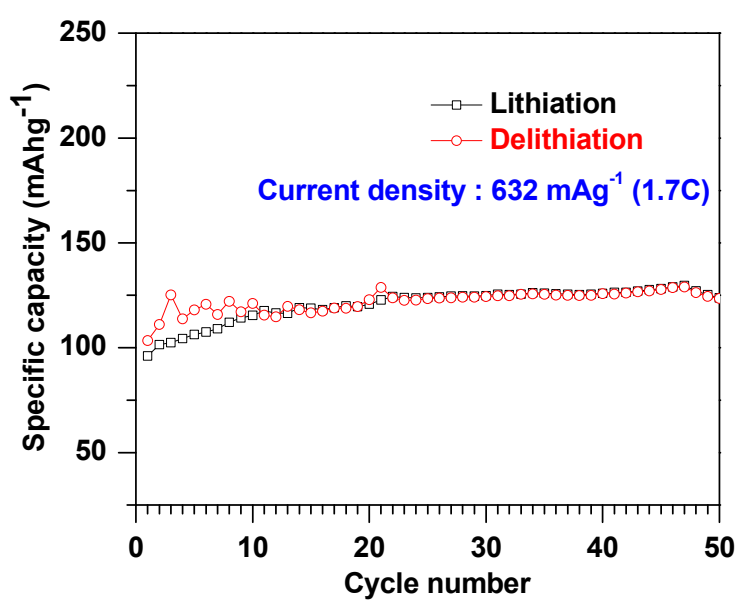

(b)

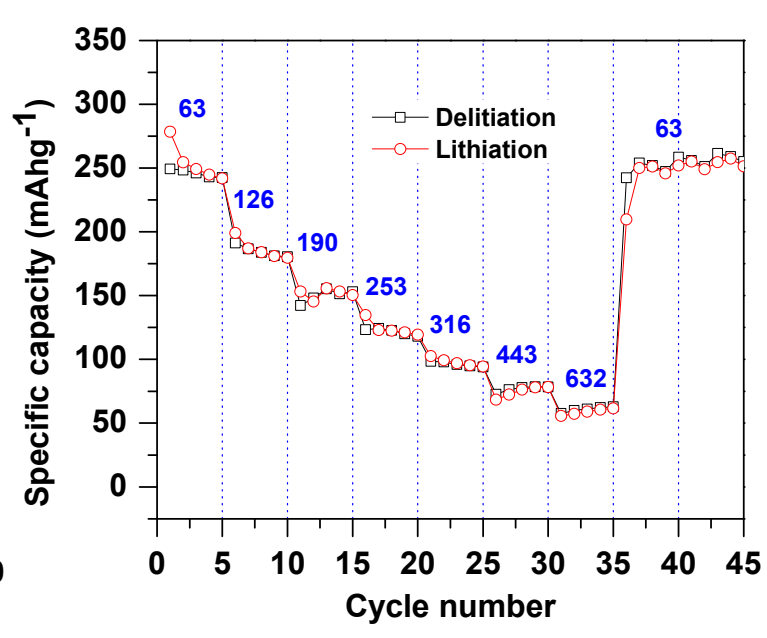

(d)

Figure 48. Electrochemical performance of the 3D MWCNTs-graphene-PET anode. (a) Initial Galvanostatic charge and discharge profiles of 3D MWCNTs-graphene-PET film anode structure, (b) Cycling performance of the anode in higher number of cycles, (c) Coulombic efficiency of the anode at $1.7 \mathrm{C}$, (d) C-rate capability of the anode at seven different current densities. Numerical values noted in (d) indicate current densities, at which charge and discharge cycles were conducted. 
Another essential performance of the LIB anodes is the rate capability. The values of specific capacities are measured at different current densities as illustrated in Figure 48(d). A typical staircase type of decreasing behavior is shown from $254 \mathrm{mAhg}^{-1}$ to $59 \mathrm{mAhg}^{-1}$ with the increase of the current densities from $0.17 \mathrm{C}$ to $1.7 \mathrm{C}$, respectively. Such trend can be presumably due to the slower rate of charge transfer reaction between $\mathrm{Li}^{+}$ions and electrons through the anode structure at the higher C-rate condition. But, the specific capacity retained to earlier $\sim 254 \mathrm{mAhg}^{-1}$ (at $0.17 \mathrm{C}$ ) at 37 th cycle even after undergoing high rate till $1.7 \mathrm{C}$ which indicates the persistence and enormous capability of withstanding different $\mathrm{C}$ rate of the flexible battery. The ability of the flexible electrode to withstand against bending angle till $\sim 180^{\circ}$, uncomplicated transfer without getting modified structural and vibrational properties and the relative good performance of the electrochemical properties divulge the effectiveness of the flexible MWCNTs-graphene-PET electrode.

\subsection{Conclusion}

We have demonstrated a novel 3D MWCNTs-graphene-PET flexible anode structure for flexible LIB. The 3D MWCNTs and graphene are directly grown on $\mathrm{Cu}$ substrates by using a CVD method and the 3D MWCNTs-graphene structure is transferred onto flexible PET film using a thermal lamination method and wet-etching process for $\mathrm{Cu}$. It is confirmed from Raman spectra that graphene and MWCNT show similar G and D peak intensity before and after their transfer over PET substrate which indicates no degradation of the crystallographic order of the MWCNTs after transferring process. Also, the $\mathrm{Cu}$ etched 3D structure contains carbon higher than $99 \%$ which is estimated from the EDS analysis. The low sheet resistance $(\sim 95 \Omega / \mathrm{sq})$ of $3 \mathrm{D}$ MWCNTs-graphene-PET even after bending the substrate and extreme connectivity indicates the flexibility of the anode. The anode structure shows high reversible specific capacity (153 $\mathrm{mAhg}^{-1}$ 
at $0.17 \mathrm{C}$ ) and good cycling stability up to the measured range of 50 cycles at $1.7 \mathrm{C}$ yielding 124 $\mathrm{mAhg}^{-1}$. Moreover, the specific capacity retains to $\sim 254 \mathrm{mAhg}^{-1}$ at 37 th cycle at $0.17 \mathrm{C}$ even after undergoing charge-discharge cycling till 1.7C. Such good electrochemical properties are strongly related to the interconnecting electronic pathways between 3D MWCNTs and graphene due to the good bonding strength between 3D MWCNTs-graphene hybrid structure and PET substrate. Considering all those features, our proposed 3D MWCNTs-graphene-PET flexible structure is expected to be a promising anode for next generation flexible Li-ion batteries. 


\section{CHAPTER 7}

\section{SUMMARY AND FUTURE WORK}

\subsection{Summary}

Recently, high-efficiency and environmentally friendly electric vehicles (EVs) have attracted strong attention from researchers and industries around the world. Among the candidate power sources for EVs, Li-ion battery (LIB) has been used due to its major advantages, such as high specific energy and volumetric energy density. Also, to enhance the LIB performance, a high quality of anode materials is required, and hence carbon based materials, silicon and transition metal oxides have been proposed for the application to EVs. However, those materials of bulk size have some limitations for the EV applications (e.g., lower specific capacity for graphite, pulverization problem for silicon, etc.). To overcome these drawbacks, many research societies have been interested in the use of nanomaterials (e.g., carbon nanotubes, graphene, silicon nanowires or nanotubes, nanoscale transition metal oxides, and so on) in anode systems. Nanomaterials have their own unique properties; including high surface area, short diffusion length of lithium ion in the nanostructure, and mechanical sustainability. In this study, we have focused on multi-wall carbon nanotube-based anode materials and the synthesis of the various 3D anode nanoarchitectures to address a critical limitation of carbon based nanomaterials, which is a low areal or volumetric density.

A 3D MWCNT-based Li-ion battery (LIB) anode has been synthesized by a two-step process of catalytic thin layer deposition through a sputtering system and direct growth of MWCNTs on $\mathrm{Cu}$ substrates via a catalytic thermal chemical vapor deposition (CVD) method. In this dissertation, we have focused on the development of the preparation methods for the various 
novel anode structures; such as, 3D MWCNTs on Cu mesh, 3D MWCNT-based anode stack, and flexible 3D MWCNTs-graphene-PET architecture. Also, their structural and electrochemical properties have been further investigated for their applications to the next-generation LIB for electric vehicles (EVs).

The 3D MWCNTs on $\mathrm{Cu}$ mesh anode structures proved their higher specific capacity, cycling stability, and C-rate efficiency in comparison to those of the MWCNTs on 2D Cu foil anodes. The higher efficiency of the 3D anodes was strongly related to the higher average mass loading density of MWCNTs, which was fourfold higher than that of the 2D anodes. The a-Si deposited onto the 3D MWCNTs/Cu hybrid anodes demonstrated the further improvement of electrochemical properties.

In addition, we proposed a prototype model for the increment of the volumetric mass density of MWCNTs as active materials for the anode system used for high-efficiency LIB. The method to increase the volumetric mass density of MWCNTs was developed with several layers of freestanding 3D MWCNT structures through a $\mathrm{Cu}$ etching process and pressing them together. Based on the LIB performance obtained from the as-prepared anode stacks, the volumetric specific reversible capacities $\left(\mathrm{C}_{\text {rev }}\right)$ increased with the areal mass density of MWCNTs in initial cycles. However, the higher number stacked MWCNTs resulted in the loss of $\left(\mathrm{C}_{\text {rev }}\right)$ in later cycles, which is associated with the greater interfacial resistance among the 3D MWCNT network during cycling. To circumvent such drawbacks, we synthesized a novel binder-assisted and hot-pressed anode stack and obtained appreciably improved LIB performance from the novel anode stack.

Finally, we have fabricated a novel 3D MWCNTs-graphene-PET anode structure for application to flexible LIBs incorporated into both existing and emerging flexible electronic devices. Such 3D MWCNTs and graphene were directly grown on Cu substrates by using a CVD method and transferred onto flexible PET film using a thermal lamination method and a $\mathrm{Cu}$ 
etching process. According to the Raman spectra, graphene and MWCNT exhibited almost no change in G and D peak intensities before and after their transfer over the PET substrate, which means no degradation of the crystallographic order during the transfer process. The low value of sheet resistance ( $\sim 95 \Omega / \mathrm{sq})$ of 3D MWCNTs-graphene-PET structure, measured after bending test, clarified the high flexibility of the anode. In addition, the anode structure showed highly reversible specific capacity $\left(153 \mathrm{mAhg}^{-1}\right)$ up to 50 cycles and good cycling performance and Crate capability. Based on those excellent results, our proposed 3D MWCNTs-graphene-PET flexible structure can be considered as a high potential anode system for next-generation flexible and light weight Li-ion batteries.

\subsection{Future Scope of This Work}

MWCNTs have been considered as promising active materials for Li-ion battery anodes. However, one of the drawbacks of MWCNT-based anodes is lower loading density of MWCNTs, which is also a common problem with other nanomaterials. To address this issue, we introduced a novel geometry of 3D $\mathrm{Cu}$ current collectors, which can provide larger surface area to accommodate higher solid loading of MWCNTs for better specific capacities, compared to that of 2D MWCNTs. Also, anode stacks assembled by several 3D MWCNT layers can be a novel approach to increase the areal or volumetric density of MWCNT-based active materials. Nevertheless, one of the drawbacks found in our proposed anode was that the value of the reversible, stable specific capacity $\left(229 \mathrm{mAhg}^{-1}\right.$ at 50 th cycle and $\left.1 \mathrm{C}\right)$ was lower than the theoretical specific capacity $372 \mathrm{mAhg}^{-1}$ of graphite-based anode. We speculate that a possible reason for the problem can be strongly related to the increase in the weight portion of the MWCNTs as active materials without their contribution to capacitance. Since the as-prepared 3D MWCNTs showed the lower degree of order in the graphene stacking structure, their internal 
structure could not be favorable to facilitated lithium ion diffusion. Thus, during the cycling process under the moderate C-rate condition, it could be hard to expect that lithium ions would be inserted or extracted in or from the innermost structure of defective 3D MWCNTs with the larger diameter (average diameter $300 \mathrm{~nm}$ ). For improving the Li-ion battery performance, future experimentation should explore the optimized morphology of the 3D MWCNTs by controlling growth conditions, such as growth temperature and time, and thickness of a catalytic Ni thin layer. In addition, analysis of EIS can be performed to generate Nyquist plots where the imaginary part of the impedance is plotted as a function of the real part over a wide range of frequencies. Also, diffusion coefficient of lithium ions $\left(\mathrm{D}_{\mathrm{Li}}\right)$ of the 3D MWCNTs with different diameters can be calculated based on the AC impedance results and the Wechselstrom ${ }^{2}$ intermittent titration method (WITT) [103-104].

As stated in Chapter 4, amorphous silicon deposited on 3D MWCNT hybrid anode structure generated better Li-ion battery performance than that of the pristine 3D MWCNTs. However, the specific capacity $280 \mathrm{mAhg}^{-1}$ at the end of 50 cycles measured at $1 \mathrm{C}$, which is much lower than that of previous reports [15-16]. It was observed that the weight of the deposited a-Si was lower than $1 \%$ of that of the MWCNTs; therefore, the a-Si thin layer did not have any major contribution except slight increase in the overall specific capacity of the anode, unlike the previous results from Si nanotubes [15] and Si nanowires [16]. To increase the loading density of $\mathrm{Si}$, the author would like to focus on incorporating silicon quantum dots into MWCNTs to create a hybrid core-shell structure with the enhanced capacity.

As mentioned in Chapter 6, the 3D MWCNTs-graphene-PET flexible anodes showed good electrochemical properties. The main reason for this is strongly related to the interconnecting electronic pathways between 3D MWCNTs and graphene due to the good bonding strength

\footnotetext{
${ }^{2}$ The German word for alternating current
} 
between 3D MWCNTs-graphene hybrid structure and PET substrate. In spite of this, the specific capacity $124 \mathrm{mAhg}^{-1}$ up to the 50 cycles at $1.7 \mathrm{C}$ is still not high enough to be applied to commercial flexible Li-ion batteries. To overcome the limitation, it is proposed to exclude the effect of the insulating nature of PET film on the slower rate of charge transfer reaction between $\mathrm{Li}^{+}$ions and electrons through the anode structure at the higher $\mathrm{C}$-rate condition. It is anticipated that fabricating a pouch-type of a full Li-ion battery cell is a good cell design to realize the high potential of our proposed 3D MWCNTs-graphene-PET film anode structure, instead of using a conventional coin type of a cell. 


\section{LIST OF REFERENCES}

[1] M. Armand, J.-M. Tarascon, Nature 451 (2008) 652-657.

[2] I. Lahiri and W.B. Choi, in: W.B. Choi, J.W. Lee, Graphene : Synthesis and Applications, Chapter 10. Graphene and Graphene-Based Materials in Solar Cell Applications, CRC Press, Boca Raton, 2012, pp. 291-312.

[3] B. Scrosati, J. Garche, J. Power Sources., 195 (2010) 2419-2430.

[4] R.A. Huggins, Advanced Batteries Materials Sciences Aspects, Preface, Springer, New York, 2010, pp. i-Xv.

[5] M. Armand, J.-M. Tarascon, Nature 451 (2008) 652-657.

[6] D. MacArthur, in: G.-A. Nazri, G. Pistoia (Eds.), Lithium Batteries: Science and Technology, Springer, New York, 2004, pp. 701-706.

[7] I. Belharouak, G.M. Koenig Jr., K. Amine, J. Power Sources., 196 (2011) 10344-10350.

[8] B. Scrosati, J. Garche, J. Power Sources., 195 (2010) 2419-2430.

[9] A.S. Aricò, P. Bruce, B. Scrosati, J.-M. Tarascon, W.V. Schalkwijk, Nature. Materials. 4 (2005) 366-377.

[10] X. X. Wang, J. N. Wang, H. Chang and Y. F. Zhang, Adv. Funct. Mater., 17 (2007) 36133618.

[11] P. Guo, H. H. Song and X. H. Chen, Electrochem. Commun., 11 (2009) 1320

[12] C.K. Chan, R. Ruffo, S.S. Hong, R.A. Huggins, Y. Cui, J. Power Sources., 189 (2009) 34-39.

[13] A. Kuhn, R. Amandi, F. Garcia-Alvarado, J. Power Sources., 92 (2001) 221-227.

[14] L.F. Nazar, O. Crosnier, Anodes and Composite Anodes: An Overview, in: G.-A. Nazri, G. Pistoia (Eds.), Lithium batteries: science and technology, Springer, New York, 2004, pp. 116-121.

[15] M.H. Park, M.G. Kim, J.B. Joo, K.T. Kim, J.Y. Kim, S.H. Ahn, Y. Cui, J.P. Cho, Nano. Lett. 9 (2009) 3844-3847.

[16] C.K. Chan, H.L. Peng, G. Liu, K. McIlwrath, X.F. Zhang, R.A. Huggins, Y. Cui, Nat. Nanotechnol. 3 (2008) 31-35.

[17] R. Ruffo, S.S. Hong, C.K. Chan, R.A. Huggins, Y. Cui, J. Phys. Chem. C 2009, 113, 1139011398.

[18] P. Poizot, S. Laruelle, S. Grugeon, L. Dupont, J-M. Tarascon, Nature 407 (2000) 496-499. 
[19] M.M. Rahman, S.-L. Chou, C. Zhong, J.-Z. Wang, D. Wexler, H.-K. Liu, Solid State Ionics 180 (2010) 1646-1651.

[20] B. Varghese, M.V. Reddy, Z. Yanwu, C.S. Lit, T.C. Hoong, G.V.S. Rao, B.V.R. Chowdari, A.T.S. Wee, C.T. Lim, C.-H. Sow, Chem. Mater. 20 (2008) 3360-3367.

[21] L.F. Nazar, O. Crosnier, Anodes and Composite Anodes: An Overview, in: G.-A. Nazri, G. Pistoia (Eds.), Lithium batteries: science and technology, Springer, New York, 2004, pp. 112-115.

[22] M. Winter, K.-C. Moeller, J.O. Besenhard, Carbonaceous and Graphitic Anodes, in: G.-A. Nazri, G. Pistoia (Eds.), Lithium batteries: science and technology, Springer, New York, 2004, pp. 144-194.

[23] R.A. Huggins, Advanced Batteries Materials Sciences Aspects, Chapter 7. Negative Electrodes in Lithium Cells, Springer, New York, 2010, pp. 123-134.

[24] M. Endo, M.S. Strano, P.M. Ajayan, Potential applications of carbon nanotubes, Carbon Nanotubes, Springer, New York, 111 (2008), pp. 13-61.

[25] P.M. Ajayan and O. Zhou, in: M.S. Dresselhaus, G. Dresselhaus, Ph. Avouris (Eds.), Carbon Nanotubes Synthesis, Structure, Properties, and Application, Springer, New York, 2001, pp. 401404.

[26] E. Frackowak, S. Gautier, H. Gaucher, S. Bonnamy, F. Beguin, Carbon, 37 (1999) 61-69.

[27] G.T. Wu, C.S. Wang, X.B. Zhang, H.S. Yang, Z.F. Qi, P.M. He, W.Z. Li, J. Electrochem. Soc., 146 (1999) 1696-1701.

[28] S. Yang, J. Huo, H. Song, X. Chen, Electrochim. Acta 53 (2008) 2238-2244.

[29] X.X. Wang, J.N. Wang, L.F. Su, J. Power. Sources. 186 (2009) 194-200.

[30] K. Lin, Y. Xu, G. He, X. Wang, Mater. Chem. Phys. 99 (2006) 190-196.

[31] J. Zhou, H. Song, B. Fu, B. Wu, X. Chen, J. Mater. Chem., 2010, 20, 2794-2800.

[32] B. Gao, A. Kleinhammes, X.P. Tang, C. Bower, L. Fleming, Y. Wu, O. Zhou, Chem. Phys. Lett. 307 (1999) 153-157.

[33] A.S. Claye, J.E. Fischer, C.B. Huffman, A.G. Rinzler, R.E. Smalley, J. Electrochem. Soc. 147 (2000) 2845-2852.

[34] I. Lahiri, S.W. Oh, J.Y. Hwang, S.J. Cho, Y.K. Sun, R. Banerjee, W.B. Choi, ACS. Nano. 4 (2010) 3440-3446.

[35] I. Lahiri, S.M. Oh, J.Y. Hwang, C.W. Kang, M.S. Choi, H.T. Jeon, R. Banerjee, Y.K. Sun, W.B. Choi, J. Mater. Chem. 21 (2011) 13621-13626.

[36] Y. Gogotsi, P. Simon, Science. 34 (2011) 917-918. 
[37] C. Arbizzani, S. Beninati, M. Lazzari, M. Mastragostino, J. Power. Sources. 141 (2005) 149155.

[38] Z. Yan, L. Ma, Y. Zhu, X. Yu, P.V. Braun, Nat. Nanotechnol. 6 (2011) 277-281.

[39] S.K. Cheah, E. Perre, M. Rooth, M. Fondell, A. Hårsta, L. Nyholm, M. Boman, T. Gustafsson, J. Lu, P. Simon, K. Edström, Nano. Lett. 9 (2009) 3230-3233.

[40] Y.Q. Zhang, X.H. Xia, X.L. Wang, Y.J. Mai, S.J. Shi, Y.Y. Tang, C.G. Gu, J.P. Tu, J. Power. Sources. 213 (2012) 106-111.

[41] L.F. Cui, Y. Yang, C.M. Hsu, Y. Cui, Nano. Lett. 9 (2009) 3370-3374.

[42] P.-C. Chen, J. Xu, H. Chen, C. Zhou, Nano. Res. 4 (2011) 290-296.

[43] L. Hu, J.W. Choi, Y. Yang, S. Jeong, F. La Mantia, L.F. Cui, Y. Cui, PNAS 106 (2009) 21490-21494.

[44] L. Hu, Y. Cui, Energy Environ. Sci., 5 (2012) 6423-6435.

[45] L. Hu, H. Wu, F. La Mantia, Y. Yang, Yi Cui, ACS. Nano. 4 (2010) 5843-5848.

[46] V.L. Pushparaj, M.M. Shaijumon, A.Kumar, S. Murugesan, L. Ci, R. Vajtai, R.J. Linhardt, O. Nalamasu, P.M. Ajayan, PNAS 104 (2007)13574-13577.

[47] J. Chen, A.I. Minett, Y. Liu, C. Lynam, P. Sherrell, C. Wang, G.G. Wallace, Adv. Mater. 20 (2008) 566-570.

[48] M. Kaempgen, C.K. Chan, J. Ma, Y. Cui, G. Gruner, Nano. Lett., 9 (2009) 1872-1876.

[49] Displaybank : Flexible Display Technology and Market Forecast (2007 - 2017) Report

[50] A. J. Salkind, A. G. Cannone, F. A. Trumbure, Lead-Acid Batteries, in Handbook of Batteries, ed. Linden, D., Reddy, T. B., 2002; 3rd edn, pp. 23.1-23.88.

[51] G. M. Ehrlich, Lithium-Ion Batteries, in Handbook of Batteries, ed. Linden, D., Reddy, T. B., 2002; 3rd edn, pp. 35.1-35.94.

[52] T. B. Reddy, S. Hossain, Rechargeable Lithium Batteries (Ambient Temperature), in Handbook of Batteries, ed. Linden, D., Reddy, T.B., 2002, 3rd edn, pp. 34.1-34.62.

[53] J.G. Park et al. Principles and Applications of Lithium Secondary Batteries, 2010, 1st edn, pp. 8.

[54] B.J. Landi, M.J. Ganter, C.D. Cress, R.A. DiLeo, R.P. Raffaelle, Energy. Environ. Sci. 2 (2009) 638-654.

[55] D. Aurbach, J. Power Sources 89 (2000) 206-218. 
[56] J. Graetz, C.C. Ahn, R. Yazami, B. Fultz, Electrochem. Solid-State Lett., 6 (2003) A194A197.

[57] M. Winter, K.-C. Moeller and J.O. Besenhard, Carbonaceous and Graphitic Anodes, in: G.-A. Nazri, G. Pistoia (Eds.), Lithium batteries: science and technology, Springer, New York, 2004, pp. 149.

[58] H. Shi, J. Barker, M.Y. Saiidi, R. Koksbang, L. Morris, J. Power Sources 68 (1997) 291-295.

[59] L.F. Nazar, O. Crosnier, Anodes and Composite Anodes: An Overview, in: G.-A. Nazri, G. Pistoia (Eds.), Lithium batteries: science and technology, Springer, New York, 2004, pp. 117.

[60] C. K. Chan, X. F. Zhang, Y. Cui, Nano. Lett., 8 (2008) 307-309.

[61] P. Meduri, C. Pendyala, V. Kumar, G. U. Sumanasekera, M. K. Sunkara, Nano Lett., 9 (2009) 612-616.

[62] H. Dai, Surf. Sci., 500 (2002) 218-241.

[63] S. Iijima, Nature, 354 (1991) 56-58.

[64] P.M. Ajayan and O.Z. Zhou, in: M.S. Dresselhaus, G. Dresselhaus, Ph. Avouris (Eds.), Carbon Nanotubes Synthesis, Structure, Properties, and Application, Springer, New York, 2001, pp. 401-404.

[65] E. Frackowiak, F. Beguin, Carbon 40 (2002) 1775-1787.

[66] O. Zhou, R.M. Fleming, D.W. Murphy, C.H. Chen, R.C. Haddon, A.P. Ramirez, S.H. Glarum, Science 263 (1994) 1744-1747.

[67] S.S. Zhang, T.R. Jow, J. Power Sources 109 (2002) 422-426.

[68] E.P. Roth, D.H. Doughty, J. Franklin, J. Power Sources 134 (2004) 222-234.

[69] I. Lahiri, W. Choi, Acta. Materialia. 59 (2011) 5411-5421.

[70] V.P. Verma, "Enhanced zinc oxide and graphene nanostructures for electronics and sensing applications", Dissertation in FIU (January 1st 2010).

[71] I. Lahiri, R. Seelaboyina, J.Y. Hwang, R. Banerjee, W. Choi, Carbon. 48 (2010) 1531-1538.

[72] I. Lahiri, D. Lahiri, S. Jin, A. Agarwal, W.B. Choi, ACS. Nano. 5 (2011) 780-787.

[73] G. Cao, Nanostructures and Nanomaterials - Synthesis, Properties and Applications, Chapter 5. Two-Dimensional Nanostructures: Thin Films, Imperial College Press, London, 2006, pp. 174175. 
[74] H. Dai, in: M.S. Dresselhaus, G. Dresselhaus, Ph. Avouris (Eds.), Carbon Nanotubes Synthesis, Structure, Properties, and Application, Springer, New York, 2001, pp. 30-34.

[75] G.L. Hornyak, J. Dutta, H.F. Tibbals and A.K. Rao, Introduction to Nanoscience, Chapter 9. Cabon-Based Nanomaterials, CRC Press, Boca Raton, 2008, pp. 469-470.

[76] R.T.K. Baker, M.A. Barber, P.S. Harris, F.S. Feates, R.J. Waite, J. Catal., 26 (1972) 51-62.

[77] V.I. Merkulov, D.H. Lowndes, Y.Y. Wei, G. Eres, E. Voelkl, Appl. Phys. Lett. 76 (2000) 3555-3557.

[78] C. Ducati, I. Alexandrou, M. Chhowalla, G.A.J. Amaratunga, J. Robertson, J. Appl. Phys. 92 (2002) 3299-3303.

[79] M.P. Siegal, D.L. Overmyer, P.P. Provencio, Precise control of multiwall carbon nanotube diameters using thermalchemical vapor deposition, Appl. Phys. Lett. 80 (2002) 2171-2173.

[80] Y. Y. Wang, S. Gupta, R. J. Nemanich, Z. J. Liu and L. C. Qin, J. Appl. Phys. 98 (2005) 014312 .

[81] C. Kang, I. Lahiri, R. Baskaran, W.-G. Kim, Y.-K. Sun, W.B. Choi, J. of Power. Sources. 219 (2012) 364-370.

[82] S.V. Lomov, L. Gorbatikh, I. Verpoest, Carbon. 49 (2011) 2079-2091.

[83] B. Gao, C. Bower, J.D. Lorentzen, L. Fleming, A. Kleinhammes, X.P. Tang, L.E. McNeil, Y. Wu, O. Zhou, Chem. Phys. Lett. 327 (2000) 69-75.

[84] H.-C. Shin, M. Liu, B. Sadanadan, A.M. Rao, J. Solid. State. Electrochem. 8 (2004) 908-913.

[85] Z. Iqbal, S. Vepiek, J. Phys. C: Solid. State. Phys. 15 (1982) 377-392.

[86] C.d.1. Casas, W. Li, J. Power Sources. 208 (2012) 74-85.

[87] G. Wang, X. Shen, J. Yao, D. Wexler, J.H. Ahn, Electrochem. Commun. 11 (2009) 546-549.

[88] Y.T. Lee, J. Park, Y.S. Choi, H. Ryu, H.J. Lee, J. Phys. Chem. B. 106 (2002) 7614-7618.

[89] S. Bhattacharya, A.R. Riahi, A.T. Alpas, J. Power Sources., 196 (2011) 8719-8727.

[90] F.Y. Zhang, A.K. Prasad, S.G. Advani, Investigation of a copper etching technique to fabricate metallic gas diffusion media, J. Micromech. Microeng. 16 (2006) N23-N27.

[91] S. Komaba, K. Shimomura, N. Yabuuchi, T. Ozeki, H. Yui, K. Konno, Study on Polymer Binders for High-Capacity SiO Negative Electrode of Li-Ion Batteries, J. Phys. Chem. C, 115 (2011) 13487-13495.

[92] A. Magasinski, P. Dixon, B. Hertzberg, A. Kvit, J. Ayala, G. Yushin, Nat Mater 9 (2010) 353-358. 
[93] J.Y. Eom, D.Y. Kim, H.S. Kwon, J. Power Sources 157 (2006) 507-514.

[94] R.A. Huggins, Advanced Batteries Materials Sciences Aspects, Chapter 7. Negative Electrodes in Lithium Cells, Springer, New York, 2010, pp. 134.

[95] R.A. Huggins, Advanced Batteries Materials Sciences Aspects, Chapter 16. Electrolyte Stability Windows and Their Extension, Springer, New York, 2010, pp. 383-387.

[96] R. Moshtev, B. Johnson, Journal of Power Sources 91 (2000) 86-91.

[97] Z.J. Fan, J. Yan, T. Wei, G.-Q.Ning, L.-J.Zhi, J.-C.Liu, D.-X.Cao, G.-L. Wang, and F. Wei, ACS Nano. 5 (2011) 2787.

[98] G. Zou, D. Zhang, C. Dong, H. Li, K. Xiong, L. Fei, Y. Qian, Carbon 44 (2006) 828-832.

[99] M. Cole, P. Hiralal, K. Ying, C. Li, Y. Zhang, K. Teo, A. Ferrari, W. Milne. J. Nanomater. Volume 2012, Article ID 272960, 8 pages.

[100] Z. Yan, L. Ma, Y. Zhu, I. Lahiri, M.G. Hahm, Z. Liu, S. Yang, C. Xiang, W. Lu, Z. Peng, Z. Sun, C. Kittrell, J. Lou, W.B. Choi, P.M. Ajayan, J.M. Tour, ACS Nano. 7 (2013) 58.

[101] V.P. Verma, S. Das, I. Lahiri, W.B. Choi, Appl. Phys. Lett. 96 (2010) 203-108.

[102] M. Liang, L. Zhi, J. Mater. Chem., 19 (2009) 5871-5878.

[103] C. Ho, I.D. Raistrick, R.A. Huggins, J. Electrochem. Soc. 127 (1980) 343-350.

[104] R.A. Huggins, Advanced Batteries Materials Sciences Aspects, Chapter 17. Experimental Methods to Evaluate the Critical Properties of Electrodes and Electrolytes, Springer, New York, 2010, pp. 406-414. 
VITA

\section{CHI WON KANG}

\section{EDUCATION}

May 2009 - present

Ph.D. scholar of Mechanical and Materials Engineering, Florida International University, Miami, FL, USA; Research topic: Development of nanostructured Materials (CNT, graphene) for Li-ion battery anodes; Advisor : Dr. Wonbong Choi; GPA : 3.93 out of 4.00

January 2007 - May 2009

M.S., Materials Engineering, Auburn University, Auburn, AL, USA; Thesis title : Structural and Electrical Characterization of Highly Oriented $\left(\mathrm{K}_{\mathrm{x}} \mathrm{Na}_{\mathrm{x}}\right) \mathrm{NbO}_{3}(\mathrm{KNN})$ Thin Films by Chemical Solution Deposition; Advisor : Dr. Dong-Joo (Daniel) Kim; GPA : 3.33 out of 4.00

August 2005 - December 2006

In pursuit of Ph.D. of Materials Science and Engineering, University of Florida, Gainesville, FL, USA; Research topic : Biomaterials; Advisor : Dr. Christopher Batich; GPA : 3.09 out of 4.00

March 2003 - July 2005

In pursuit of Ph.D. of Materials Science and Engineering, Gyeongsang National University, Jinju, South Korea; Research topic : Electronic materials; Advisor : Dr. Youngjae Shim

March 2001 - February 2003

M.S., Ceramic Engineering, Yonsei University, Seoul, South Korea; Thesis title : Fabrication of Barrier Rib of Plasma Display Panel (PDP) by Transfer Molding of Gelable Slurry; Advisor : Dr. Jooho Moon; GPA : 3.69 out of 4.30

March 1999 - February 2001

B.S., Materials Science and Engineering, Yonsei University, Seoul, South Korea; GPA : 3.40 out of 4.30

March 1997 - February 1999

In pursuit of B.S. of Metallurgy and Materials Engineering, Hanyang University, Ansan, South Korea;

GPA : 3.99 out of 4.50

\section{JOURNAL PUBLICATIONS}

1. C.W. Kang, R. Baskaran, W.B. Choi, "Large Scale Patternable 3-Dimensional Carbon Nanotubes-Graphene Structure for Flexible Li-ion Battery", submitted to Carbon (under review).

2. C.W. Kang, I. Lahiri, R. Baskaran, W.-G. Kim, Y.-K. Sun, W.B. Choi, "3-dimensional carbon nanotube for Li-ion battery anode”, J. Power Sources. 219 (2012) 364-370.

3. I. Lahiri, S.M. Oh, J.Y. Hwang, C.W. Kang, M.S. Choi, H.T. Jeon, R. Banerjee, Y.K. Sun, W.B. Choi, "Ultrathin alumina-coated carbon nanotubes as an anode for high capacity Li-ion batteries", J. Mater. Chem. 21 (2011) 13621-13626. 
4. S. Das, I. Lahiri, C.W. Kang, W.B. Choi, "Engineering carbon nanomaterials for future applications: energy and bio-sensor", Society of Photo-Optical Instrumentation, 868 (2011) 93.

5. I. Lahiri, S. Das, C.W. Kang, W.B. Choi, "Application of carbon nanostructures - Energy to electronics", JOM, 63 (2011) 70-76.

\section{PATENT}

1. W. Choi, I. Lahiri, C. Kang, High efficiency lithium ion battery anode based on 2- and 3dimensional carbon nanotube-metal/alloy substrates, US Provisional Patent Appl. No. 61567979 (Filed on December 7, 2011).

\section{INTERNATIONAL CONFERENCE PROCEEDINGS}

1. C.W. Kang, R. Baskaran, W.-G. Kim, Y.-K. Sun, W.B. Choi, "3-Dimensional Carbon Nanostructures for Li-ion Battery Anode," 2012 MRS Fall Meeting, Volume 1505 (2013), Symposium W - Carbon Nanomaterials.

2. C.W. Kang, I. Lahiri, R. Baskaran, M.S. Choi, W.-G. Kim, Y.-K. Sun, W.B. Choi, "3D Multiwall Carbon Nanotubes (MWCNTs) for Li-Ion Battery Anode," TMS 2012 Supplemental Proceedings: Materials Properties, Characterization, and Modeling, Volume 2, pp. 35-41.

\section{INTERNATIONAL CONFERENCE PRESENTATIONS}

1. R. Baskaran, C.W. Kang, J.G. Park, H.T. Jeon, W.B. Choi, "Surface modified MWCNT by ALD coated $\mathrm{RuO}_{2}$ anode for Li-ion battery", NANO KOREA 2013, July 10-12, 2013, Seoul, Republic of Korea.

2. C.W. Kang, R. Baskaran, W.-G. Kim, Y.-K. Sun, W.B. Choi, "3-Dimensional Carbon Nanostructures for Li-ion Battery Anode”, 2012 MRS Fall Meeting, November 25-30, 2012, Boston, Massachusetts, USA.

3. R. Baskaran, C.W. Kang, I. Lahiri, W.B. Choi, "Ex-situ investigations on 3D MWCNT anode for Li-ion battery", NANO KOREA 2012, August 16-18, 2012, Seoul, Republic of Korea.

4. C.W. Kang, I. Lahiri, R. Baskaran, M.S. Choi, W.-G. Kim, Y.-K. Sun, W.B. Choi, "3D Multiwall Carbon Nanotubes (MWCNTs) for Li-Ion Battery Anode", 2012 TMS Annual Meeting \& Exhibition, March 11-15, 2012, Orlando, Florida, USA.

5. C.W. Kang, I. Lahiri, R. Baskaran, M.S. Choi, Y.-K. Sun, W.B. Choi, “An efficient Li-Ion Battery Anode Based on 3D Carbon Nanotubes Structure", NanoFlorida ${ }^{\mathrm{TM}}$ 2011, September 30 October 1, 2011, Miami, Florida, USA.

6. C.W. Kang, I. Lahiri, Y.-K. Sun, W.B. Choi, "Synthesis and electrochemical characterization of carbon nanostructures for Li-ion battery anode", UKC 2010, US-Korea Conference on Science, Technology, and Entrepreneurship, August 11-15, 2010, Seattle, Washington, USA. 